\title{
Developmental effects of oxytocin neurons on social affiliation and processing of social information
}

Ana Rita Nunes ${ }^{1,2, *}$, Michael Gliksberg ${ }^{2, *}$, Susana A.M. Varela ${ }^{1,3}$, Magda Teles $^{1}$, Einav Wircer $^{2}$, Janna Blechman², Giovanni Petri ${ }^{4}$, Gil Levkowitz ${ }^{2, \#}$, Rui F. Oliveira ${ }^{1,3,5, \#}$

* These authors contributed equally to the work

${ }^{1}$ Integrative Behavioural Biology Lab, Instituto Gulbenkian de Ciência, Oeiras 2780-156, Portugal.

2 Department of Molecular Cell Biology, Weizmann Institute of Science, Rehovot 7610001 , Israel.

${ }^{3}$ ISPA- Instituto Universitário, Lisboa 1149-041, Portugal

${ }^{4}$ ISI Foundation \& ISI Global Science Foundation, 10126 Torino, Italy

${ }^{5}$ Champalimaud Research, Champalimaud Centre for the Unknown, 1400-038 Lisbon, Portugal.

\# Correspondence should be addressed to

G.L. gil.levkowitz@weizmann.ac.il

R.F.O. ruiol@ispa.pt

Abbreviated title: Oxytocin effects on development of the social brain

30 pages; 6 main figures; 14 extended data figures.

Abstract- 174 words; Introduction- 505 words; Discussion- 782 words

KEYWORDS: Sociality, oxytocin, dopamine, zebrafish, development, organizational hypothesis, social decision making network 


\section{Conflict of interest: The authors declare no competing financial interests ACKNOWLEDGMENTS}

We thank all members of Gil Levkowitz's and Rui Oliveira's laboratories for fruitful discussions, and Nitzan Konstantin for English editing. We thank the technical support of IGC's Advanced Imaging Facility (AIF-UIC), which is supported by the national Portuguese funding ref\# PPBI-POCI-01-0145-FEDER-022122, co-financed by Lisboa Regional Operational Programme (Lisboa 2020), under the Portugal 2020 Partnership Agreement, through the European Regional Development Fund (FEDER) and Fundação para a Ciência e Tecnologia (FCT, Portugal); all the staff from the Fish Facility platforms of Instituto Gulbenkian de Ciência, Portugal, and Weizmann Institute of Science, Israel, for animal care and valuable advice; Congento, which is supported by the funding ref\# LISBOA-01-0145FEDER-022170, co-financed by Lisboa Regional Operational Programme (Lisboa 2020), under the Portugal 2020 Partnership Agreement, through the European Regional Development Fund (FEDER) and Fundação para a Ciência e a Tecnologia (FCT; Portugal); IGC's Histopathology Facility for technical support, help and valuable advices; the Advanced Biolmaging and BioOptics Experimental Platform (ABBE Platform) of the Champalimaud Center for the Unknown (CCU) for all the technical support and help. 


\section{Abstract}

Hormones regulate behavior either through activational effects that facilitate the acute expression of specific behaviors or through organizational effects that shape the development of the nervous system thereby altering adult behavior. Much research has

5 implicated the neuropeptide oxytocin (OXT) in acute modulation of various aspects of social behaviors across vertebrate species, and OXT signaling is associated with the developmental social deficits observed in autism spectrum disorders, however, little is

8 known about the role of OXT in the neurodevelopment of the social brain. We show that perturbation of OXT neurons during early zebrafish development led to a loss of dopaminergic neurons, associated with visual processing and reward, and blunted the neuronal response to social stimuli in the adult brain. Ultimately, adult fish whose OXT neurons were ablated in early life, displayed altered functional connectivity within social decision-making brain nuclei both in naïve state and in response to social stimulus and became less social. We propose that OXT neurons have an organizational role, namely to shape forebrain neuroarchitecture during development and to acquire an affiliative response towards conspecifics.

\section{Significance Statement:}

18 Social behavior is developed over the lifetime of an organism and the neuropeptide oxytocin modulates social behaviors across vertebrate species, and is associated with neuro-developmental social deficits such as autism. However, whether oxytocin plays a

21 role in the developmental maturation of neural systems that are necessary for social

22 behavior remains poorly explored. We show that proper behavioral and neural response

23 to social stimuli depends on a developmental process orchestrated by oxytocin neurons.

24 Animals whose oxytocin system is ablated in early life show blunted neuronal and 25 behavioral responses to social stimuli as well as wide ranging disruptions in the functional 26 connectivity of the Social Brain. We provide a window into the mechanisms underlying 27 oxytocin-dependent developmental processes that implement adult sociality. 


\section{INTRODUCTION}

Social behavior describes any of a wide group of behaviors by an organism towards members of its species, termed conspecifics (Robinson et al., 2019). The systems underlying these behaviors are thought to be established during development whereas the behavioral manifestations themselves are not immediately put into effect, but become apparent only later in life.

The "organizational hypothesis" claims that hormones can shape the structure of the developing nervous system and as a result alter the adult animal behavior and was first suggested by Phoenix, et al. following their study of the developmental effect of testosterone exposure on subsequent sexual behavior (Phoenix et al., 1959). Organizational effects refer to long-term, irreversible impact of hormones on tissue differentiation that can either directly or indirectly influence physiology, metabolism and behavior. In the context of behavior, organizational signals that can be mediated by neuropeptides, are necessary during critical developmental windows in order to shape neural systems whose activity will only become relevant at a later stage in the lifetime of the animal. This organizational effect is contrasted with the acute or "activational" effect exerted by hormones, neurotransmitters and neuropeptides on behavior, physiology and metabolism.

The neuropeptide oxytocin (OXT) has a widely-studied activational role in several aspects of social behavior (for a review see (Donaldson and Young, 2008; Grinevich et al., 2016; Jurek and Neumann, 2018)), including social processing (Gamer et al., 2010; Grinevich and Stoop, 2018), attention (Bartz et al., 2011; Lukas et al., 2011; ShamayTsoory and Abu-Akel, 2016), reward (Insel and Shapiro, 1992; Bowen and Neumann, 2017), and pro-social behaviors (Lukas et al., 2011), in rodents and humans. Previously, we showed that this activational role of OXT is evolutionarily conserved in deep time,

53 since OXT also modulates perception of visual social cues (Nunes et al., 2020) and social

54 recognition (Ribeiro et al., 2020a, 2020b) in zebrafish.

OXT is thought to have organizational effects as well. As early as 1989, Noonan et al. 
57 on behavior in the adult (Noonan et al., 1989). In a more recent example, Keebaugh and

58 Young utilized selective viral overexpression to modulate OXTR levels in the nucleus

59 accumbens of pre-pubertal female prairie voles and showed that this developmental

60 intervention is necessary and sufficient to induce alloparental behavior in the adult

61 (Keebaugh and Young, 2011). Such results have been described in various mammalian

62 models, behavioral domains, and using pharmacological (e.g. peptide, agonist and

63 antagonist injection) as well as genetic interventions in prenatal and prepubertal animals

64 (for a review see (Miller and Caldwell, 2015) and (Hammock, 2015)). Furthermore, human

65 studies of Autism Spectrum Disorder (ASD) have strongly suggested that certain features

66 of ASD arise from very early developmental abnormalities which are thought to be linked

67 to OXT signaling, further suggesting an organizational role for OXT in the development

68 of social behavior (Heinrichs et al., 2009; Hovey et al., 2014) or for a review (Guastella

69 and Hickie, 2016). However, the mechanisms underlying this organizational mode of

70 action have thus far remained elusive.

71 It has been well established that oxytocin communicates with other neurotransmitter

72 systems for proper social behaviors. Dopamine (DA) is mainly involved in reward and

73 reinforcement systems (Dölen and Malenka, 2014; Love, 2014), but also in sensory

74 modulation and attention gating (Love, 2014; Grinevich et al., 2015). DA neurons are

75 activated during social interactions and mating, and interact with the OXT system to

76 promote pair-bond formation (Dölen and Malenka, 2014; Johnson et al., 2017) and

77 sociability (Hung et al., 2017), suggesting that OXT and dopamine are both necessary to

78 promote the sensory and rewarding aspects of social interactions.

79 Here, we show that in zebrafish, signaling by OXT neurons is required in an

80 organizational manner during early life for the display of social affiliation in adulthood.

81 Furthermore, we go on to show that perturbing this process in a very early developmental

82 time window, equivalent to fetal developmental stages in mammals, has long-lasting

83 effects on specific dopaminergic clusters associated with reward and visual attention/

84 processing, and also leads to wide-ranging changes in brain activity within previously

85 described social processing networks. 
MATERIALS AND METHODS

\section{Experimental model}

Zebrafish were raised and bred according to standard protocols. Zebrafish were kept in mixed sex groups (10 adults/l) in a recirculation life supporting system (tecniplast) with the following parameters: $28^{\circ} \mathrm{C}$, pH 7.0, conductivity $1000 \mu \mathrm{S} / \mathrm{cm}, 14 \mathrm{~L}: 10 \mathrm{D}$ light:dark cycle. Fishes were fed with a combination of live food (Paramecium caudatum and Artemia salina) and commercial processed dry food (Gemma). Husbandry protocols, water chemistry and health program have been described previously (Borges et al., 2016). All experimental procedures were conducted in accordance with standard operating procedures of the Instituto Gulbenkian de Ciência and Direcção Geral de Alimentação e Veterinária (DGAV- Direcção Geral de Alimentação e Veterinária, permit number 0421/000/000/2015), Portugal, and Institutional Animal Care and Use Committee (IACUC, protocol number 27100516) of the Weizmann Institute, Israel.

Zebrafish transgenic/mutant lines used in this study: $T g(o x t: E G F P)(B l e c h m a n$ et al., 2011), Tg(oxt:Gal4)wz06 (Anbalagan et al., 2018), Tg(UAS:NTR-mCherry)c264 (Davison et al., 2007), $T g(U A S: s y p b-E G F P)$ (Meyer and Smith, 2006). Larvae and adult zebrafish (3-6 months old) of both sexes were used in this work.

\section{Social affiliation assay}

106 The social preference test followed the protocol described previously (Wircer et al., 2017; 107 Ribeiro et al., 2020a). Briefly, focal zebrafish were given a choice between two side-byside compartments: one containing a shoal (two males and two females) and an empty one (Figure 1A-B) during a 10-min test. The stimulus shoal matched the genotype of the

110 focal fish. The stimulus compartment was randomly switched between tests, to control for 111 any place preference possibly induced by the arena or laboratory frames. All 112 compartments were completely sealed to block transmission of chemical and vibrational 113 stimuli and thus, only visual cues were accessible. The experimental test tank was placed 114 over a custom-built infrared LED light box, to increase image quality for subsequent 
automated video tracking. Fish behavior was recorded from above with either a highspeed camera FLARE (2M360, lo Industries) or a B\&W mini surveillance camera

117 (Henelec 300B) connected to a computer, using video recording software (Pinnacle

118 Studio 12). Videos were analyzed with Ethovision XT11.0 (Noldus Inc., The Netherlands).

119 Relevant data were then exported and further analyzed. The two regions of interest, 120 empty and shoal, were defined as the 1/10 of the length of the arena immediately adjacent 121 to the empty compartment or the compartment containing the stimulus shoal, 122 respectively. The percentage of cumulative time fish spent in these regions of interest $123\left(\% \mathrm{~T}_{\text {shoal }}\right.$ and $\left.\% \mathrm{~T}_{\text {empty }}\right)$ was used to calculate the social affiliation score $\left[\% \mathrm{~T}_{\text {shoal }} /\left(\% \mathrm{~T}_{\text {shoal }}\right.\right.$ $\left.\left.124+\% \mathrm{~T}_{\text {empty }}\right)\right]$. This score, also called the sociability score, has been commonly used in 125 rodent studies to measure sociability in the 3-chamber test (Park et al., 2018). Total 126 distance traveled was also measured.

127 Ontogeny of social affiliation behavior: Social preference was tested at different 128 developmental stages: early larvae (12 days-old), mid larvae (14-20 days-old), post129 flexion metalarvae (21-30 days-old), juveniles and adults (from 30 to 90 days-old). The 130 size of the testing arena for early larvae was $3.5 \times 6.0 \mathrm{~cm}$; for mid larvae $7.0 \times 8.0 \mathrm{~cm}$; for 131 metalarvae $7.0 \times 10.0 \mathrm{~cm}$; and for juveniles and adults it was $20.0 \times 20.0 \mathrm{~cm}$. The stimulus 132 shoal matched the developmental stage of the focal fish. Two different experiments were 133 performed: a) longitudinal, where individual fish were repeatedly tested at each 134 developmental time point, and b) cross-sectional, where individual fish were tested only 135 once, at a given developmental time point.

136 From all behavioral experiments, animals were excluded from analysis if they failed to 137 enter both the ROls (i.e. Shoal and Empty) as this results in a social score equals to 138 infinity.

141 Early ablation: Nitroreductase (NTR)-mediated cellular chemically induced OXT 142 neuronal ablation was conducted as previously described(Curado et al., 2007, 2008). 143 Briefly, larvae ( 30-40 per petri dish) were treated with metronidazole (MTZ, Vetranal 144 CAT\#46461, Sigma) dissolved in Danieau buffer to a final concentration of 10mM for 48 
$145 \mathrm{~h}$ while being protected from light, to prevent MTZ photoinactivation. After the first $24 \mathrm{~h}$, 146 the MTZ-containing Danieau buffer was replaced by fresh MTZ medium. Control 147 untreated larvae were subjected to the same procedure in Danieau buffer without MTZ, 148 but were also protected from light. After $48 \mathrm{~h}$ of treatment, larvae were washed out several 149 times in Danieau buffer.

150 Adult ablation: We used a modified ablation protocol of 3 X48 h MTZ treatments (5 mM), 151 as we observed that this increased survival of adult animals. Briefly, adult zebrafish were 152 placed into 2-liter tanks containing MTZ (Veterinary preparation, Vetmarket; Shoham, 153 Israel, CAT\#165228, or 2-methyl-5-nitroimidazole-1-ethanol, TCL Europe, CAT\#M0924) 154 dissolved in system water at a final concentration of $5 \mathrm{mM}$, protected from light. After $\sim 16$ $155 \mathrm{~h}$ of treatment, zebrafish were moved to a tank containing fresh system water without $156 \mathrm{MTZ}$, fed, and allowed to recover for $\sim 2 \mathrm{~h}$, followed by a second treatment of MTZ 5mM 157 for $16 \mathrm{~h}$. Animals were then allowed to recover in their home tanks for $48 \mathrm{~h}$. The entire 158 protocol was repeated three times.

159 Effect of chemically induced OXT neuronal ablation on endogenous OXT cells. 160 Zebrafish treated with MTZ at different time points during development (4-6 days-old, 1216114 days-old, 20-22 days-old or 90 days-old) were allowed to recover from treatment for $16248 \mathrm{~h}$ and were then sacrificed to assess the treatment effects on endogenous OXT cells 163 by in situ hybridization (see below) or by direct visualization of the mCherry transgene 164 signal. OXT cells were manually counted using imageJ software (Schindelin et al., 2012), 165 except for in situ experiment at 22-24 days-old where we used a semi-automated method for quantifying total fluorescence (see below). neuronal ablation. Zebrafish larvae were ablated at 12-14 DPF, and then allowed to grow until adulthood. At 90 DPF animals were sacrificed, brains harvested, and subjected 170 to fluorescent in situ hybridization (see below). The total fluorescence was then quantified 171 in a semi-automated manner (see below).

172 Effect of chemically induced OXT neuronal ablation on adult social affiliation. 173 Zebrafish treated with MTZ at 4-6 days-old, 12-14 days-old, 20-22 days-old or 90 days- 
174 old were allowed to grow until adulthood to be tested for social affiliation behavior (see 175 above).

Time course of NTR-mCherry recovery following 4-6 days-old chemically induced OXT neural ablation. Fish were treated with MTZ at 4-6 days-old and sampled at different time points: 8, 12, 19, 26 and 42 days-old.

Semi-automated method for quantifying total fluorescence. For quantification of images in the recovery experiment, and the effect of ablation at 22-24 DPF on OXT mRNA we utilized a semi-automated method for quantifying total fluorescence. Each Z-stack was broken into its component optical planes, and ROl's were selected for the region containing the cells and the background (a region within the tissue with no fluorescent cells). The threshold for "positive" pixels was defined as the 75th percentile value of al the pixels in the background ROI, and all positive pixels per slice were counted. the total number of positive pixels per sample was summed, and then normalized to the maximal value for that cohort. Source code in MATLAB for this procedure is included.

Effect of 4-6 days-old chemically induced OXT neuronal ablation on adult neuronal activity (pS6 activation). To assess the effect of early OXT ablation on adult brain activation patterns in response to a social stimulus, adult zebrafish that had been treated with MTZ at 4-6 days-old and untreated controls were exposed individually to either a mixed-sex shoal of conspecifics or an empty tank for $10 \mathrm{~min}$. Immediately after, we blocked the visual stimulus, without disturbing the focal fish, by placing an opaque partition between the experimental and stimulus tanks. After 50 min (to allow for expression of the pS6 neural activation marker), zebrafish were sacrificed and heads were collected and processed for paraffin slice immunofluorescence (see below).

Effect of 4-6 days-old chemically induced OXT neuronal ablation on larvae and adult dopaminergic system. Untreated and 4-6 days-old MTZ-treated zebrafish larvae were allowed to recover from treatment (48h) and were either processed immediately for whole-mount larvae TH immunofluorescence (see below) or allowed to grow until adulthood and then processed for paraffin slice TH-immunofluorescence (see below). 


\section{In situ hybridization}

204 RNA in situ hybridization was performed on both whole larvae and whole adult brains.

205 Larvae or dissected brains were fixed in 4\%PFA and in situ hybridization was performed 206 as described in (Machluf and Levkowitz, 2011; Wircer et al., 2017). An OXT probe was 207 generated using a pGEM plasmid encoding for oxt mRNA (RefSeq NM_178291.2). 208 Following development with Fast Red reagent (Roche, cat. No. 11496549001), adult 209 brains were embedded in agar and sagittally cut at a thickness of $150 \mu \mathrm{m}$ on a vibratome, 210 and whole larvae and brain slices were mounted in glycerol and imaged on a Zeiss LSM 211800 scanning confocal microscope.

\section{Whole-mount larvae immunofluorescence}

214 Briefly, larvae were euthanized in ice-cold water, transferred to 4\% PFA and then 215 incubated overnight at $4^{\circ} \mathrm{C}$ on a shaker. Then, the PFA was washed out and samples 216 were placed in pre-cooled acetone in a freezer at $-20^{\circ} \mathrm{C}$ for $10 \mathrm{~min}$. The acetone was 217 washed out (PBS 0.1\% TritonX-100), and the samples were then incubated in blocking 218 solution (PBS $0.1 \%$ triton $+1 \%$ DMSO $+1 \% \mathrm{BSA}+5 \% \mathrm{NGS}$ ) for minimum of $2 \mathrm{~h}$ at room 219 temperature (RT), followed by incubation with primary antibody overnight at $4^{\circ} \mathrm{C}$ on a 220 shaker. Primary antibodies used were either mouse anti-TH (MAB 318, Merck Millipore), 221 rabbit anti-EGFP (A11122, ThermoFisher) or guinea pig anti-OXT (T-5021, Peninsula 222 labs), at a concentration of 1:200. Next, samples were washed repeatedly (minimum of 2236 X15 min washes) with blocking solution and then placed in a blocking solution containing 224 fluorescent secondary antibody $(1: 200)$ overnight at $4^{\circ} \mathrm{C}$ on a shaker. Then, samples were 225 washed in PBS, mounted dorsally on a slide in mounting medium (Aqua-Polymount, 226 Polysciences Inc., Warrington PA, CAT\# 18606-20) and imaged by a Zeiss LSM 800 227 scanning confocal microscope.

\section{Paraffin adult brain slice immunofluorescence}

230 Zebrafish heads were fixed in $10 \%$ buffered formalin for $72 \mathrm{~h}$ and decalcified in EDTA $231(0.5 \mathrm{M}, \mathrm{pH} 8.0)$ for $48 \mathrm{~h}$, followed by paraffin inclusion. Coronal slices ( $6 \mu \mathrm{m}$-thick) were 
232 cut with a microtome. Sectioned slices were then processed for immunofluorescence.

233 After antigen retrieval with Tris-EDTA (10 mM Tris Base, $1 \mathrm{mM}$ EDTA, 0.05\% Tween20)

234 at $95^{\circ} \mathrm{C}$ for $20 \mathrm{~min}$, slices were washed in TBS-Tx (TBS 0.025\% TritonX-100, 3X10 min),

235 then incubated with blocking solution (TBS $0.025 \%$ TritonX-100 + 1\%BSA) for $1 \mathrm{~h}$ at RT,

236 followed by an overnight incubation with a primary antibody $\left(1: 400\right.$ at $\left.4^{\circ} \mathrm{C}\right)$. Slides were

237 then washed in TBS- 0.025\% TritonX-100 (3X10 min) and incubated in secondary

238 antibody (1:1000 for $2 \mathrm{~h}$ ). After washes, slices were incubated with DAPI for 20 min, then

239 rinsed in TBS and mounted with EverBrite ${ }^{\mathrm{TM}}$ Hardset Mounting medium (Biotium).

240 Primary antibodies used were anti-phosphorylation of S6 ribosomal protein, pS6 (Cell

241 signaling S235/236) and anti-tyrosine hydroxylase, anti-TH, (mouse monoclonal, MAB

242 318, Merck Millipore).

\section{Neuronal quantifications}

\section{Neuronal pS6 cell quantification}

245 Coronal images were acquired with a commercial Nikon High Content Screening 246 microscope, based on Nilon Ti equipped with a Andor Zyla 4.2 sCMOS camera, using a $24720 \times 0.75$ NA objective, quadruple dichroic filter, and controlled with the Nikon Elements 248 software. To avoid double-imaging of the same cells, we imaged every other slice. We 249 quantified density of pS6-positive cells in 16 brain areas that belong to the social decision 250 making network (O'Connell and Hofmann, 2011): Vv- ventral nucleus of the ventral 251 telencephalic area (V), homologous to the mammalian lateral septum, subdivided into: 252 anterior ( $\left.\mathrm{V} \mathrm{v}_{\mathrm{a}} \mathrm{a}\right)$ and posterior $\left(\mathrm{V} \mathrm{v}_{-} \mathrm{p}\right)$; $\mathrm{Vd}$ - dorsal nucleus of $\mathrm{V}$, homologous to the 253 mammalian Nacc, subdivided into: anterior (Vd_a), medial (Vd_m) and posterior (Vd_p);

254 Vc- central nucleus of V, homologous to the mammalian striatum, subdivided into: anterior 255 (Vc_a) and posterior (Vc_p); Dm- medial zone of the dorsal telencephalic area (D), 256 homologous to the mammalian bLAMY; DI- lateral zone of $\mathrm{D}$, homologous to

257 hippocampus; Dd- dorsal zone of D; Vs- supracommissural nucleus of V, homologous to 258 the mammalian extended amygdala (BNST and meAMY); Vp- postcommissural nucleus 259 of V; Ppa- parvocellular preoptic nucleus, anterior part and Ppp- parvocellular preoptic 260 nucleus, posterior part, both homologous to the mammalian preoptic area; VM261 ventromedial thalamic nucleus; VL-ventrolateral thalamic nucleus (Figure 6-1). 
262 For each brain area, about five coronal brain slices were analyzed manually using imageJ 263 software (Schindelin et al., 2012). Background subtraction and linear adjustments of 264 brightness and contrast levels were performed in the same way for all groups. The brain 265 areas of interest were identified using DAPI to define neuroanatomical boundaries and 266 landmarks identified in the Zebrafish Atlas (Wullimann and Mueller, 2004). In each brain 267 slice, we placed one square of $1000 \mu \mathrm{m}^{2}$ in the brain region of interest, in each 268 hemisphere, following these criteria: the square was always placed over the highest 269 number of pS6-positive cells, keeping minimum distance from the border and edge of the 270 brain section, a similar strategy that has been used by others (Lorenzi et al., 2017). pS6271 positive cells were counted if a nucleus surrounded by the cytoplasm was clearly visible, 272 and if the intensity of the pS6 signal was perceptibly greater than background (see Figure 2736 for examples of pS6 staining in the sampled brain areas).

\section{Dopaminergic neuronal quantification}

276 Dopaminergic quantification was performed in both larvae and adult zebrafish, either 4-6 277 days-old MTZ-treated or untreated. Briefly, for larvae, whole-mount zebrafish 278 immunofluorescence was performed as described above using primary antibody anti-TH. 279 Larvae were imaged and confocal z-stacks were analyzed by ImageJ. Larva 280 dopaminergic forebrain, pretectum and posterior tuberculum nucleus were identified 281 according to (Sallinen et al., 2009; Tay et al., 2011) and TH+ cells were counted in these 282 three groups.

283 For adults, heads from both treatment groups (untreated and 4-6 days-old MTZ-treated) 284 were collected and processed for paraffin embedding, followed by paraffin slice 285 immunofluorescence as described above. Coronal slices were acquired with a 286 SlideScanner Zeiss AxioScan Z1 (Zeiss), and analyzed with Zeiss Zen Lite software. To 287 avoid double-imaging of the same cells, we imaged every other slice. Boundaries 288 separating brain nuclei and subdivisions were identified based on DAPI staining, using as 289 reference a coronal atlas of the zebrafish (Wullimann et al., 1996). Dopaminergic neurons $290\left(\mathrm{TH}^{+}\right)$were identified using the same criteria as for $\mathrm{pS6}{ }^{+}$neurons. 
$291 \mathrm{TH}^{+}$nuclei groups were identified according to (Rink and Wullimann, 2001; Panula et al.,

292 2010; Parker et al., 2013) (Figure 4-1). We counted $\mathrm{TH}^{+}$cells in the following clusters: the 293 subpallium area (extending from the rostroventral telencephalon to the dorsocaudal

294 telencephalon, also identified as G2 by Panula and colleagues (Panula et al., 2010), 295 which included a TH cluster containing Vv, Vc and VI, and in-between areas, a TH cluster 296 including Vd and the lateral area outside the Vd, and a TH cluster within the Vs (identified 297 by the anterior commissure) and lateral area outside the Vs; TH clusters in the PPa area 298 were divided in two: a more anterior (close to the border of the diencephalon, lateral 299 margin of the ventricle, G3) and a more posterior extending area in the medial area of the $300 \mathrm{PPa}$ (ventral part of the PPa), G4); a TH cluster in the pretectum (PPr, G7); in the 301 ventromedial and ventrolateral nucleus (Vm, VI, G6); a $\mathrm{TH}$ cluster in the periventricular 302 nucleus of the posterior tuberculum (TP/TP, small cells, ventral to the central posterior 303 thalamic nucleus (CP), G11) and in the posterior tuberal nucleus (PTN, G12) (Figure 4304 1). Similar to pS6 neuronal quantification described above, we manually analyzed five 305 coronal brain slices for each cluster, in each brain hemisphere, placing one square of $1000 \mu \mathrm{m}^{2}$ in the brain region of interest with the highest density of cells.

\section{Statistical analysis}

308 Data are represented as mean \pm standard error of the mean (SEM). Normality of the data was tested by D'Agostino and Pearson omnibus normality test and Shapiro-Wilk normality tests. When parametric assumptions were verified, we used parametric statistics; otherwise we used equivalent non-parametric tests, namely Mann-Whitney tests to compare the effect of MTZ treatment on OXT mRNA, on the expression of the

313 NTR-mCherry transgene, and to compare the adult social affiliation score between 314 untreated and MTZ-treated groups at different developmental time points (4-6 days-old, 315 12-14 days-old, 22-24 days-old and 90 days-old MTZ-treatment). Significance was 316 denoted as $p<0.05$, and $p$-values refer to two-tailed tests, unless otherwise noted. The 317 use of one-tailed tests was justified by a priori directionality hypotheses in the following 318 cases: 
- Effect of MTZ treatment on OXT mRNA and NTR-mCherry transgene expression (Figure 2 and Figure 2-1), since in our system, we expected that MTZ would ablate the NTRoxytocinergic expressing neurons;

- Effect of MTZ treatment (oxytocinergic ablation) on social affiliation behavior in fish expressing the NTR transgene, and thus causing OXT ablation, since OXT is well known to regulate social behaviors and thus, we expected that oxytocinergic ablation would lead to a decreased social behavior in zebrafish (Figure 2).

Statistical analysis of behavioral data: One sample $t$-tests were used to verify if social affiliation during development was statistically different from chance (0.5). Because we tested eight independent cohorts for the effect of MTZ-treatment at 4-6 days-old time point (by different researchers in two different laboratories), the total sample size for this time point $(n=210)$ was much larger than for the other time points (12-14 days-old: $n=28,22-$ 24 days-old, $n=19$; adult: $n=13$ ). Taking in account all eight cohorts together, the adult social affiliation score of the 4-6 days-old MTZ-treated fish were significantly decreased from untreated siblings $(p=0.0012$, Mann-Whitney test, $n=112$ untreated vs. 98 MTZtreated fish). However, a Generalized Linear Model (GLM) with beta regression and planned comparisons, which was used to compare the effect of MTZ treatment on adult social affiliation score of the eight different cohorts (Figure 2-3), revealed not only an effect of the MTZ treatment $\left(F_{1}=9.486, p=0.0021\right)$ but also variation among cohorts $\left(F_{7}=2.719, p=0.0081\right.$, Figure 2-4). We therefore performed a Monte Carlo simulation for a sample size of 30 individuals sampled from the observed data, 15 individuals from each group (untreated and 4-6 days-old MTZ treated) with 1000 iterations. This simulation, ensured a more comparable sample size for the 4-6 days-old time point treatment relative to other time-point treatments. Moreover, it ensured a more representative sample than each cohort independently or the total eight-cohort population. We obtained a Monte

344 Carlo $p$-value of $p<0.001$ (Figure 2E), which means that there was a significantly higher

345 number of simulations in which the social affiliation score of the untreated group was greater than the social affiliation score of the MTZ-treated group, as compared to the number of simulations with the inverse trend, for a sample size of 15 individuals, across all eight cohorts. P-value was calculated as the mean social affiliation score of the 1000 
349 iterations for untreated fish divided by the mean scores of the 1000 iterations for treated 350 fish divided by 1000.

351 To determine whether the difference in social affiliation score between untreated and 4-6 352 days-old MTZ-treated fish was due to OXT ablation and not movement impairments 353 caused by MTZ, we used a Linear Model (LM) to compare the total distance moved 354 (square root transformed) of both groups in the eight independent cohorts. Both GLM with 355 beta regression on adult social affiliation score and LM on total distance moved (square 356 root transformed) were also applied to different control cohort fish not expressing NTR 357 transgene (Figure 2-3, 2-5). In all of these models (GLM and LM) the explanatory 358 variables were treatment and cohorts for mains effects and interaction.

359 Statistical Analysis of neuronal activation (ps6 cell quantification): To compare neuronal activation between treatments and stimuli, we used a Generalized Linear Mixed Model (GLMM) with a Poisson distribution using the brain identification number as the random effect in the statistical model, followed by planned comparisons, since we target areas that are known to belong to the social decision-making network. We checked whether the data were normally distributed with Shapiro-Wilk tests for each brain area. Since they were not, and because the data are counts, we checked the fit of the data to the Poisson distribution with the "fitdistrplus" package from R (Delignette-Muller and Dutang, 2015). By visual inspection of density and cumulative distribution plots we validated that the Poisson distribution was the most appropriate to our data analyses. As

369 fixed effects we used treatment and stimuli. We analyzed the 16 brain areas 370 independently and corrected the p-values of the planned comparison with the false 371 discovery rate (FDR) adjustment method.

372 Statistical Analysis of dopaminergic cell quantification: To compare dopaminergic 373 cell numbers between treatments, and since the data were normally distributed (G7: W = $3740.97685, \mathrm{p}$-value $=0.6383 ; \mathrm{G} 12: \mathrm{W}=0.9546, \mathrm{p}$-value $=0.1459 ; \mathrm{G} 2: \mathrm{W}=0.95265, \mathrm{p}-$ 375 value $=0.1267$; Shapiro-Wilk test), LM was performed to assess the effect of 4-6 days376 old MTZ-treatment in total TH counts of three distinct independently analyzed TH clusters 377 in 8 days-old larvae (Figure 3, 3-1). 
378 The effect of 4-6 days-old MTZ treatment on adult dopaminergic system was also 379 assessed with a GLMM with a Poisson regression followed by planned comparisons, comparing the number of TH-positive cells sampled in five squares of $1000 \mu \mathrm{m}^{2}$ per $\mathrm{TH}$ cluster in 8 different adult brain TH clusters. As fixed effects we used treatment and stimuli. All eight areas were analyzed independently and p-values of the planned comparisons were corrected with the FDR adjustment method (Figure 4 and 4-2).

Data analysis software: Graphical representations of the data were performed in GraphPad Prism 6.0c software. Shapiro-Wilk normality test, one-sample $t$-test and $t$-tests were performed in GraphPad Prism, and the remaining tests were performed in $R$ programming software, version 3.6.3 (R_core_Team, 2013) with the following packages: Ime4(Bates et al., 2015) and afex (Henrik Singmann, Ben Bolker, Jake Westfall, 2020) (for GLMM with Poisson regression), betareg (for GLMM with beta regression), emmeans (for planned comparisons)(Lenth, 2020), and the base R package (for LM regression and Monte Carlo simulations)(R_core_Team, 2013).

\section{Functional connectivity analysis}

393 Functional connectivity, which has been defined as the temporal coincidence of spatially distant neurophysiological events (Friston, 1994), is typically inferred from temporal correlations between distinct brain areas in human fMRI data from both resting state and task-state studies (Biswal et al., 1997). The rationale for this approach is that areas that show a consistent correlation in their activity are components of the same brain network.

398 Therefore, the concept of functional connectivity is purely correlative in nature, and per se it does not imply a direct causal relationship or the occurrence of structural connectivity between the correlated brain areas (Eickhoff and Müller, 2015). Functional connectivity can also be inferred from correlations across subjects using the expression of molecular markers such as immediate early genes (Teles et al., 2015). Similarly, we computed

403 functional connectivity for our neuronal activity data based on the co-expression of pS6 404 across different brain regions for the set of individuals of each experimental treatment. 405 For this purpose we have developed the method described below, which statistically 406 validates the identified networks. 
407 Network reconstruction: To test for functional connectivity of early MTZ-treated vs 408 untreated brains exposed to social stimuli, we reconstructed Pearson correlation matrices 409 using a resampling procedure, similar to the Quadratic Assignment Procedure (QAP) 410 (Makagon et al., 2012; Borgatti et al., 2013). Instead of defining a single network, we 411 construct the set of possible networks obtained leaving out some of the specimens' 412 information. More precisely, consider the case of $M$ specimens, each with an associated 413 expression vector $x_{i} \in \mathbb{R}^{N}$, where $N$ is the number of brain regions. For a given $m<M$, 414 we consider all the $\left(\begin{array}{c}M \\ m\end{array}\right)$ combinations $\Omega_{\gamma}$ of $m$ specimens and compute the corresponding 415 functional graphs. We refer to the collection of graphs obtained in this way as a graph 416 tower $\Omega_{\Gamma}$, where each of the combinations can be considered as a graph layer. The 417 advantage of this construction is that each layer in the graph tower represents a different 418 instance of the network bootstrapping. In this way, observables computable on a single 419 layer can be bootstrapped across multiple ones. The construction has naturally one 420 parameter, the sampling number $m$, which needs to be chosen on the basis of data-driven 421 considerations. In our experiments, robustness analysis shows that results are robust for $422 m$ values between 10 and 15. To obtained sparse functional connectivity matrices, we 423 threshold each instance following (De Vico Fallani et al., 2017) at a density threshold of $424 \rho=0.2$, which also corresponds to the minimum network heterogeneity across instances. 425 After thresholding, for each treatment we average the thresholded instances to obtain a 426 single matrix per treatment, which we use in the following analysis.

427 Detection of robust functional modules: Communities were computed using the 428 Leiden community detection method (Traag et al., 2019) on the averaged treatment 429 matrices. To increase the robustness of the detection, for each condition, we repeat the 430 community detection 400 times. From the 400 candidate partitions we extract the central 431 partition as described in (Peixoto, 2021) and associate the resulting central partition to 432 the treatment under analysis. To quantitatively characterize differences among partitions, 433 we measure the ratio $r$ of total edge weights within a community with that of the edges 434 between communities. More specifically, for a partition $\mathcal{P}$ with $s$ communities we compute 435 the $s \times s$ matrix $P$, defined as 


$$
P_{\alpha \beta}=\sum_{i \in \alpha, j \in \beta} \omega_{i j}
$$

437 Where $\alpha, \beta=0, \ldots m-1$ label the modules of $\mathcal{P}$ and $\omega_{i j}$ is the edge weight between

438 regions $i$ and $j$. We then compute the ratio of average intra-community to inter-community 439 edge weights as follows:

$$
r=\frac{(m-1) \sum_{\alpha} P_{\alpha \alpha}}{2 \sum_{\alpha \neq \beta} P_{\alpha \beta}}=
$$$$
\frac{(m-1)}{2} \frac{\operatorname{Tr} P}{|P|_{1}-\operatorname{Tr} P}
$$

442 which measures the ratio of the average weight on the diagonal of $P_{\alpha \beta}$ to the average 443 diagonal weight. To assess significance of $r$ values and of differences between them, we 444 employ a permutation test based on permutating the community labels while preserving 445 the size of the considered communities. We find that all $r$ values are significantly different 446 from zero, and so are also the differences in $r$ between treatments $(p<0.01)$.

447 Strength centrality: As a measure of local integration, we computed also the strength 448 centralities. The first encodes how strongly a node links to its neighbors. while the second 449 total measures how influential a node is at the network level on the basis of its direct 450 connections and how well connected its neighbors are. In Figure 6-4 we report the nodes 451 ranked in decreasing order of strength (weighted degree) centrality. 


\section{RESULTS}

\section{Early life ablation of oxytocinergic neurons decreases social affiliation}

Zebrafish is a highly gregarious species exhibiting well-characterized social behaviors (reviewed in (Nunes et al., 2017)). We quantified the visually-mediated motivation of zebrafish to approach conspecifics, as an indicator of social affiliation, by performing a two-choice preference test measuring the time that individuals spend either near a compartment containing a shoal of conspecifics or an empty one (Ribeiro et al., 2020a, $2020 b)$. When an adult zebrafish is visually presented with both shoal and non-shoal compartments, in a side-by-side configuration, it will spend most of the time in association with the shoal; however, if shoal is not visible, fish tend to explore the entire arena (Figure1 A,B). In accordance with previous reports (Engeszer et al., 2007; Dreosti et al., 2015), we found social affiliation is an acquired developmental trait that emerges after the third week of life. This was observed both in zebrafish that were repeatedly exposed to the social affiliation behavioral arena throughout development (12 days-old $(d): p=0.69$, $n=15 ; 14 d: p=0.91, n=14 ; 16 d: p=0.73, n=15 ; 16 d: p=0.88, n=17 ; 22 d: p=0.049, n=12$; 24d: $p=0.07, n=18 ; 26 d: p=0.01, n=17 ; 28 d: p=0.057, n=13 ; 30 d: p=0.17, n=8 ; 33 d:$ $p<0.0001, n=14 ; 62 d: p=0.002, n=10$; one sample t-test vs. theoretical score of 0.5 indicating no preference, Figure 1C), and in zebrafish that were only exposed to the arena once at a specific developmental time point to avoid habituation to the setup $(12 d: p=0.34$, $n=16 ; 19 d: p=0.48, n=18 ; 21 d: p=0.85, n=6 ; 26 d: p=0.76, n=10 ; 36 d: p=0.0007, n=13$; 90d: $p<0.0001, n=24$; one sample t-test vs. theoretical score of 0.5 indicating no preference, Figure 1C).

Oxytocin (OXT) has long been known to regulate social behaviors across species (Goodson, 2008) and it has been linked to neurodevelopmental disorders that impact social behavior during development and adulthood (Pobbe et al., 2012; Hovey et al., 2014). This makes OXT a good candidate system for investigating neurodevelopmental 480 processes linked to social development. To disentangle the organizational vs. activational 481 effects of OXT neurons in the acquisition of adult social affiliation, we used a transgenic line, Tg(oxt:Gal4;UAS:NTR-mCherry), to express nitroreductase (NTR) protein fused to 
483 the mCherry reporter, in oxytocin neurons (Figure 1D,E). In the presence of the drug 484 metronidazole (MTZ), NTR produces cytotoxic metabolites, thereby allowing temporally 485 controlled ablation of OXT neurons at different developmental stages (Curado et al., $4862007,2008)$. We confirmed the expression of the NTR-mcherry fusion protein in the OXT487 ergic neuronal population by co-localizing the Tg(oxt:Gal4;UAS:NTR-mCherry) with a 488 transgenic reporter $\operatorname{Tg}(\mathrm{oxt}: E G F P)$ at different stages of development (8, 14, 24 and 90 489 days-old, Figure 1E).

490 In both mammals and fish, OXT is produced by specific cells in the preoptic 491 hypothalamus which can be classified as magnocellular or parvocellular, based on soma 492 size (Sawchenko and Swanson, 1982; Van den Dungen et al., 1982; Knobloch and 493 Grinevich, 2014; Grinevich et al., 2016). We and others have previously shown that 494 zebrafish magnocellular OXT cells are clustered in the anterior-dorsal portion of the cell 495 group, whereas the parvocellular neurons make up the more ventral part of the group 496 (Wircer et al., 2017; Wee et al., 2019). We observed that the NTR-mCherry protein was 497 prominently expressed in the most anterior-dorsal part of the preoptic area (Figure 1E), 498 thus it can be considered to localize mainly to magnocellular OXT neurons in both larvae 499 and adult zebrafish. These cells have been shown to project to ventral forebrain in fish 500 (Saito et al., 2004). Also, recent research in rats has shown that these magnocellular cells 501 project to several brains regions including hypothalamus, amygdala, lateral septum and 502 nucleus accumbens (Zhang et al., 2021), indicating that this projection pattern is 503 evolutionarily conserved. MTZ treatment at all time points was highly effective $(>80 \%$ 504 decrease) at ablating NTR-mCherry-positive OXT neurons (Figure 2-1). To ensure proper 505 ablation of endogenous OXT-ergic cells following MTZ treatment, we also performed 506 fluorescent in situ hybridization with probes directed against oxt mRNA at 4-6 days-old, 507 12-14 days-old, 22-24 days-old and in the adult (Figure 2A-C and Figure 2-2). A significant 508 decrease in OXT-expressing cells was observed following MTZ treatment at all tested 509 ages $(p=0.0024 n=11 / 10, p=0.0003 n=7 / 7, p=0.0011 n=7 / 8, p=0.0079 n=5 / 4$, for 4-6 510 days-old, 12-14 days-old, 22-24 days-old and adult ablation untreated/treated, 511 respectively, one tailed Mann-Whitney test, Figure 2A'-C' and Figure 2-2). 
To assess the effects of temporal OXT neuronal ablation on adult social affiliation, 513 zebrafish were treated with MTZ at specific time-points during development (4-6, 12-14, 514 22-24 days-old) or post-puberty (90 days-old) and social affiliation was assessed in 515 adulthood in comparison to untreated siblings (Figure 2D). We analyzed the social 516 affiliation scores of eight independent cohorts in which OXT neurons were ablated at 4-6 517 days days-old and found that despite considerable variation among cohorts during the 518 test, there was a robust effect of MTZ treatment on adult social affiliation (cohorts: $p=$ 5190.0081 ; treatment: $p=0.001$, see Statistical analysis section for detailed statistics of the 520 Generalized Linear Model (GLM) with beta regression, Figure 2-3, Figure 2-4). MTZ 521 treatment did not affect overall fish locomotion as measured by the total distance traveled 522 during the trial (cohorts: $p=8.342 e-16$, treatment: $p=0.668$, Linear Model (LM), Figure 2523 3, Figure 2-4). We also observed variation among control adult cohorts not expressing 524 NTR but treated or untreated with MTZ at 4-6 days-old, without a main effect of MTZ525 treatment on either social affiliation score (cohort: $p=0.0001$, MTZ-treatment: $p=0.2653$, 526 GLM with beta regression, Figure 2-3, Figure 2-5) or total distance moved (cohort: $p<2 e-$ 16, MTZ-treatment: $p=0.1536$, LM, Figure 2-3, Figure 2-5).

In view of the biological variability observed across cohorts, we further verified the effect of early-life OXT-neuron ablation on social affiliation by utilizing a Monte Carlo (MC) simulation where we randomly sampled 15 treated and 15 untreated 4-6 days-old larvae from all 8 cohorts and repeated the procedure for 1000 iterations. This analysis also 532 clearly indicated an impairment of adult social affiliation $(p<0.0001$, one-tailed Mann533 Whitney test, Figure 2E, see Statistical analysis for details on MC), which was maintained 534 in 12-14 days-old MTZ-treated adult fish $(p=0.034, n=14 / 14$, one-tailed Mann-Whitney 535 test, Figure 2E).

In contrast to the deficit in social affiliation caused by early life MTZ treatment, ablation 537 of OXT neurons in either 22-24 days-old or 90+ days-old animals did not affect adult 538 social affiliation (22-24: $p=0.1364, n=10 / 9$ one-tailed Mann-Whitney test; Adults: $539 p=0.3566, n=6 / 7$, one-tailed Mann-Whitney test, Figure 2E). This result coincides with the 540 observation that social affiliation is a developmentally acquired trait which is established 541 before the juvenile stage of development (Figure 1C and (Dreosti et al., 2015). 
Taken together, these results show that early developmental ablation of OXT neurons

543 leads to a long-term deficit in social affiliation in adulthood, suggesting that OXT neurons

544 are involved in an early-life developmental process that is required for proper social

545 affiliation later in life.

546 Early life OXT ablation leads to impairments in specific dopaminergic clusters

547 Notably, although early-life OXT neuronal ablation induced an impairment in social 548 affiliation, we observed complete recovery of the OXT neural population by adulthood, as 549 ablated fish displayed normal counts of OXT-expressing NTR-mCherry transgene (4-6 550 days-old MTZ-treated $(n=14)$ vs. untreated $(n=15)$ adult zebrafish: $p=0.33$, unpaired t551 test, Figure 2F-H). We verified this result, obtained from imaging of the NTR-mCherry 552 transgene, by fluorescent in situ to OXT mRNA in adult fish subjected to early-life ablation, 553 and found no significant difference from controls (MTZ-treated $(n=4)$ vs. untreated $(n=5)$ 554 adult zebrafish: $p=0.25$, two-tailed Mann-Whitney $U$ test, Figure 2-7). We performed a 555 time-course analysis for the OXT neural recovery and we found that already within 1 556 month (i.e. by 42 days-old) from the MTZ-treatment there is no discernible difference 557 between treated and control animals (8 days-old, $p<0.0001, n=15 / 15 ; 12$ days-old, $558 \mathrm{p}<0.0001, \mathrm{n}=13 / 7 ; 19$ days-old, $\mathrm{p}<0.0001, \mathrm{n}=10 / 9 ; 26$ days-old, $\mathrm{p}=0.0048, \mathrm{n}=12 / 11 ; 42$ 559 days-old, $p=0.59, n=9 / 10$; Unpaired t test with Welch's correction, Figure 2-6). This is in 560 line with previous work showing that zebrafish neurons are capable of regenerating 561 following lesions even in adulthood (Kizil et al., 2012). Conversely, animals in which OXT 562 neurons were ablated in adulthood displayed normal social drive (Figure 2E) despite the 563 fact that the ablated cell populations had not yet recovered by the time of the behavioral 564 testing (Figures 2C, 2-1).

565 We therefore hypothesized that the developmental organization of the dopaminergic 566 system that also regulates social affiliation, might have been affected by early OXT 567 ablation. Therefore, we examined whether early perturbation of the OXT neuronal system 568 affected specific tyrosine hydroxylase (TH)- positive DA-ergic neuronal clusters (Figures 569 3, 4 and 4-1), which in zebrafish are anatomically discernible from other TH-positive 570 catecholaminergic cells (Ma, 1994a, 1994b, 1997; Rink and Wullimann, 2001, 2002), and 
571 whether these effects persist over the long term. We observed that already 24 hours after 572 ablation of OXT neurons at 4-6 days-old, there was a significant decrease in 573 dopaminergic neuronal counts in the pretectum (PrT; $p=0.009, n=18 / 18, L M$, Figure 3B,

574 Figure 3-1), and the large neurons of the posterior tuberculum (TP; $p=0.0045, n=18 / 18$, 575 LM, Figure 3C, Figure 3-1). In contrast, no differences were found in telencephalic DA 576 neurons of the ventral subpallium area (Figure 3-1). The pretectum is the teleostean 577 homologue of the superior colliculus, an area known to be involved in gaze control and 578 possibly attention in zebrafish (Antinucci et al., 2019) as well as in mammals (Krauzlis et 579 al., 2004). The posterior tuberculum is the source of the ascending DA system, which is 580 considered analogous to the mammalian ventral tegmental area (VTA) (Rink and 581 Wullimann, 2001), which is involved in reward and reinforcement (Morales and Margolis, 582 2017).

583 Unlike the ablated OXT cells, which recovered over time, the deficits in DA neurons 584 observed in early OXT-ablated larvae persisted through adulthood, as adult fish that had 585 been treated with MTZ between 4-6 days-old displayed a reduced number of TH-positive 586 DA cells in the PrT ( $p=0.005, n=7 / 7$; Figure 4B, Figure 4-2, GLMM). Similarly, OXT 587 ablation caused a decrease in the DA-ergic neurons residing in two subdivisions of the 588 TP, the periventricular nucleus of the posterior tuberculum (TP) and the posterior tuberal 589 nucleus (PTN), which are distinguishable only in the adult (TP $p=0.025, n=7 / 7 ; P T N p=$ 590 0.005, n=7/7; Figure 4C-D, Figure 4-2, GLMM with Poisson regression, planned 591 comparisons and FDR corrections).

To demonstrate that at the time of their ablation, OXT neurons physically interact with 593 the affected DA neurons, we examined whether OXT neurons form synapses on DA-ergic 594 clusters of the PrT and TP (Figure 5). We employed a double transgenic line, 595 Tg(oxt:Gal4;UAS:Sypb-EGFP), which genetically expresses the synaptic marker, 596 synaptophysin-GFP in OXT neurons (Anbalagan et al., 2019), and visualized synaptic 597 contacts onto TH positive DA-ergic neurons (Figure 5). To ensure that these are indeed 598 bona-fide OXT releasing synapses, we also co-stained these fish with an anti-OXT 599 antibody (Blechman et al., 2018; Anbalagan et al., 2019) (Figure 5C-D). We found OXT 600 projections forming multiple synapses onto the DA neurons of the PrT and the TP (Figure 
$6015 C, D 1, D 2)$. We also observed OXT cells directly abutting the TH-positive cells of the TP 602 (Figure 5D3,D4), raising the possibility that OXT affects nearby DA-ergic cells in a non603 synaptic manner as has been shown in other species (Ludwig and Leng, 2006; Son et 604 al., 2013). These results indicate that these two systems, DA and OXT, are already linked 605 at early developmental stages and that early ablation of OXT neurons irreversibly impairs 606 the development of subsets of DA neurons which are key for social affiliation later in life. processing

In view of these results, we next examined whether these long-lasting developmental changes also manifested in the neural processing response to social stimuli in adults, focusing on vertebrate social decision-making network and mesolimbic reward system

612 (O'Connell and Hofmann, 2011). To this end, early-life ablated (MTZ-treated at 4-6 daysold) adult zebrafish were exposed to a single visual social stimulus (a shoal of conspecifics comprised of 2 females and 2 males) or an empty tank for controls, for 10

615 minutes, and their forebrain neuronal activity state was analyzed by immunostaining of 616 phosphorylated ribosomal protein S6 (pS6), a known correlate for neuronal activation

617 ((Knight et al., 2012), Figure 6). We then quantified the number of pS6-positive neurons 618 in several specific brain areas known to be implicated in neural processing of social 619 information and social reward (O'Connell and Hofmann, 2011) (Figures 6, 6-1, 6-2). 620 Results showed that early OXT ablation significantly affected neuronal activity in 621 response to social stimulus in two specific areas: the anterior part of the parvocellular preoptic nucleus (Ppa) and in the most anterior part of the ventral nucleus of ventral 623 telencephalon (Vv) (GLMM with Poisson regression, planned comparisons and false 624 discovery rate (FDR) corrections, Figures 6B-E, 6-2).

625 Specifically, we found that while unablated fish exhibited increased activity in the Ppa 626 upon exposure to a shoal of conspecifics, this was not observed in early ablated fish 627 (untreated shoal vs untreated empty, $p=0.024, n=11 / 7$ respectively; MTZ-treated shoal 628 vs MTZ-treated empty, $p=0.88, n=10 / 6$ respectively; Figure 6B,C, Figure 6-2). Vv neurons 629 of unablated fish exhibited decreased activity upon exposure to a social stimulus, 
630 whereas early ablated animals maintained their neuronal activity regardless of the 631 stimulus (untreated shoal vs untreated empty, $p=0.024, n=11 / 7$ respectively; MTZ-treated 632 shoal vs MTZ-treated empty, $p=0.88, n=11 / 7$ respectively; Figure 6D,E; Figure 6-2).

633 These results show that these two brain areas display deficits in neuronal response to 634 a social stimulus in early OXT-ablated zebrafish. Importantly, the $\mathrm{Vv}$ is considered analogous to the mammalian lateral septum (LS), while the Ppa is analogous to the 636 mammalian preoptic hypothalamus (POA) (Wullimann and Mueller, 2004; O'Connell and 637 Hofmann, 2011). These areas are core nodes of the social behavior network in all vertebrate species and have a strong reciprocal connection with each other (Wullimann and Mueller, 2004; O’Connell and Hofmann, 2011).

We next examined whether early-life ablation of OXT neurons in the POA altered functional connectivity between forebrain nuclei belonging to the social decision-making network (SDMN; (O'Connell and Hofmann, 2012)), by comparing the correlation matrices

643 of activity levels for the different areas between ablated and non-ablated fish in either 644 basal state or in response to social stimulus (Figure 6-3, 6-4). We constructed correlation 645 matrices corresponding to each treatment via a resampling scheme, inspired by 646 Quadratic Assignment Procedure (Makagon et al., 2012), that additionally allows to 647 choose the threshold for sparsification on the basis of minimal heterogeneity across 648 resampled correlation matrices (see Methods). To identify functional reconfigurations 649 between different treatments, we identified mesoscale differences in the way the 650 functional networks are organized by their modular (or community) structure. To extract 651 such modules, we performed community detection using the Leiden algorithm (Traag et 652 al., 2019) on the functional connectivity matrix of each treatment. To ensure the 653 robustness of the resulting partitions, we repeated the optimization 400 times per 654 treatment and reconciled the different candidate partitions by considering the central 655 partition (Peixoto, 2021) (see final partitions in Figure 6F). Already by visual inspection it 656 is possible to observe differences in the overall community structure between treatments 657 in the allocation of nodes to communities, and in the relative integration within and across 658 communities. We quantified such integration by calculating the ratio $r$ of the total edge 659 weights within communities to the total edge weights between different communities (see 
660 Methods). We found that in the basal state (i.e. when no visual stimulus was presented) 661 the mesoscale functional organization is different between ablated $(r=4.6)$ and non662 ablated fish ( $r=7.5$ ), implying that the ablation of OXT neurons in the POA modifies the 663 resting state networks of the forebrain SDMN (Figure 6F). In particular, ablated fish show 664 a much less segregated network with respect to non-ablated ones. Secondly, we 665 observed that, in response to the social stimulus (i.e. sight of a conspecific shoal), the 666 functional network of non-ablated fish transitions from a segregated state $(r=7.5)$ to a 667 more integrated one $(r=3.7)$, whereas ablated fish showed the opposite pattern, moving 668 from a more integrated basal state $(r=4.6)$ to a more segregated response network $669(r=6.4)$. All $r$ values are significantly different from zero, and also significantly different 670 from each other $(p<0.01)$. Finally, we completed the network analysis focusing on local 671 differences in terms of network centralities. In particular, we looked at the strength of 672 nodes, representing how strongly a node is connected to its neighbors (Figure 6-4). We 673 found a strong and significant anti-correlation between the ranking of strengths for the 674 ablated versus non-ablated fish in response to the social stimulus (Untreated Shoal vs 675 MTZ-treated Shoal, Spearman $r=-0.5, p=0.01$ ), implying that the hub nodes for non676 ablated fish are poorly connected nodes for the ablated and vice versa. Specifically, in 677 untreated fish exposed to the social stimulus, the most connected nodes were the 678 posterio-ventral part of the ventral telencephalon $\left(\mathrm{V}_{\mathrm{p}}\right)$, followed by the medial zone of 679 the dorsal telencephalic area $(\mathrm{Dm})$, two regions that have been implicated in the 680 regulation of social interactions and motivated behavior in zebrafish (von Trotha et al., 681 2014; Stednitz et al., 2018). In contrast, both these nodes drop their centrality in the 682 SDMN network of MTZ-treated fish exposed to the social stimulus (from $1^{\text {st }}$ to $5^{\text {th }}$ and from $6832^{\text {nd }}$ to $16^{\text {th }}$, among 16 nodes, respectively), where the anterio- dorsal part of the ventral 684 telencephalon $\left(\mathrm{Vd}_{\mathrm{a}}\right)$ and the lateral part of the ventral telencephalon (VI), two poorly 685 connected nodes in untreated fish (ranking $14^{\text {th }}$ and $16^{\text {th }}$, out of 16 nodes, respectively), 686 were the most connected nodes (Figure 6-4).

These results indicate that early-life OXT ablation leads to blunted response to shoal688 induced changes in brain activity within specific nuclei ( $\mathrm{V}$ and Ppa), and to a wide- 
ranging alteration in network connectivity spanning several nuclei of the social decisionmaking network in both basal state and in response to social stimulus.

Taken together, our results show that proper response to social stimuli depends on orchestrated co-development of OXT and DA neurons. We show that during development, OXT has important organizational effects. Early developmental perturbation of OXT neurons led to reduced attraction towards conspecifics, impaired the neurodevelopment of specific DA-ergic neurons, caused a blunted neural response to social stimuli in the forebrain (Ppa and Vv), and altered the connectivity of the SDMN.

\section{DISCUSSION}

Previous research has implicated OXT in various developmental disorders that affect social function in humans, have highlighted its importance in social behavior in animals, and indicated the importance of its communication with other systems, such as DA, serotonin and estrogen for proper social behavior (Heinrichs et al., 2009; Hovey et al., 2014; Grinevich et al., 2015; Grinevich and Stoop, 2018; Rajamani et al., 2018). However, there has been limited investigation into the mechanistic aspects of OXTs developmental functions, and how they are linked to its well-described social roles.

Here we utilize temporally controlled perturbations of OXT-ergic cells at different developmental timepoints during zebrafish life to show that OXT has a distinct developmental function, namely to enable proper development of specific downstream DA-ergic clusters known to be involved in visual attention gating and reward (O'Connell and Hofmann, 2011; Antinucci et al., 2019). We also show that in animals where this developmental process was perturbed, the response to social stimuli is affected, and the tendency to shoal with conspecifics is reduced.

712 The developmentally affected DA-ergic clusters, namely the pretectum (superior 713 colliculus) and the TP (VTA) have been linked in other organisms to social functioning at 714 the level of behavior and, at the molecular level, to the OXT system. Thus, in primates 715 and humans, which like zebrafish are highly dependent on vision for gathering social 716 information, OXT receptors are expressed in the superior colliculus (Schorscher-Petcu et 
717 al., 2009; Freeman et al., 2014a, 2014b, 2018), and OXT modulates the gaze and 718 oculomotor responses controlled by this nucleus (Leknes et al., 2013; Kret and De Dreu, 719 2017). This is also true of the VTA, where OXT receptors are expressed, which functions 720 to promote sociability (Hung et al., 2017). Notably, rhesus monkeys subjected to early 721 social deprivation displayed a reduction in DA neurons of the VTA (Martin et al., 1991), 722 and mouse $\mathrm{KO}$ of the autism-associated gene Nlgn3 resulted in impaired OXT signaling 723 in DA-ergic neurons and aberrant activity in the DA-ergic neurons of the VTA (Hörnberg 724 et al., 2020). This further shows how early perturbations to the OXT system can lead to wide-ranging and varied effects on other socially relevant systems in the brain. Whether 726 the changes in DA-ergic neurons, caused the observed changes in social affiliation and 727 neural activity is yet to be directly tested.

In addition to the DA-ergic deficits, we show that in normal fish, neurons of the Ppa 729 (analogous to the mammalian VTA) respond to social stimuli by increased activity, while 730 neurons of the $\mathrm{Vv}$ (analogous to the mammalian LS) respond by reducing their activity. 731 In contrast, in developmentally perturbed animals these areas do not change their activity 732 following presentation of the stimulus. Interestingly, the OXTR in the mammalian LS have 733 been implicated in the regulation of social fear (Guzmán et al., 2013; Menon et al., 2018). 734 In zebrafish, the Vv/LS has been functionally associated with social affiliation, mainly 735 through its cholinergic neurons (Stednitz et al., 2018). However, it is interesting to note 736 that in many animal models (e.g. rodents, teleosts, and cartilaginous fish), including 737 zebrafish, the Vv/LS has been shown to contain mainly GABAergic neurons, and to be a 738 source of GABAergic input to the VTA, a putative homologue of the TP, encompassing 739 the TP and PTN, in zebrafish (O'Connell and Hofmann, 2011), and to the POA (O'Connell 740 and Hofmann, 2011; Vega-Quiroga et al., 2018). Thus, it is possible that the observed 741 reduced activity in Vv/LS corresponds to GABAergic neurons that project to the Ppa and 742 TP. These areas are core nodes of the social behavior network in all vertebrate species 743 and have a strong reciprocal connection with each other (Wullimann and Mueller, 2004; 744 O'Connell and Hofmann, 2011). This suggests that social stimuli could promote a 745 disinhibition in these target areas, which would be associated with the rewarding and/or 746 motivational component of social affiliation. As a corollary, in early-ablated animals, where $747 \quad \mathrm{~V}$ activity remains high in response to social stimuli, the TP would remain inhibited, and 
748 the rewarding component of social affiliation would be attenuated, leading to decreased

749 display of this behavior.

750 In addition, we show that the overall coactivation patterns of forebrain nuclei involved 751 in social processing are altered in developmentally perturbed animals, both at baseline 752 and following presentation of a social stimulus. In other words, we show that the 753 connectivity between the different nodes of the social decision-making network changes 754 as a result of early life OXT ablation. We submit that the proper maturation of this network 755 at the level of neural architecture is dependent on early-life signaling by OXT neurons.

756 In summary, our results demonstrate that OXT neurons are required for the 757 developmental acquisition of social affiliation and exert an organizational effect on DA758 ergic neuronal populations. Furthermore, our results suggest that this role involves 759 orchestrating the co-development and maturation of several brain systems, such as the 760 midbrain social visual processing and attention systems (the pretectum), social decision761 making areas in the forebrain and ascending dopamine centers associated with reward.

762 When the OXT-dependent developmental process is perturbed, neural responses to 763 social stimuli in these regions become compromised, important parts of the social 764 decision-making network fail to synchronize their activity, and eventually, the propensity 765 to spend time near a shoal of conspecifics is reduced. 


\section{Funding:}

768 ARN was supported by a Short-Term EMBO fellowship (ASTF 420-2013), by the

769 Weizmann's Dean of Faculty postdoctoral fellowship at the Weizmann Institute, Israel,

770 and by Fundação para a Ciência e Tecnologia (FCT; SFRH/BPD/93317/2013) at

771 Instituto Gulbenkian de Ciência, Portugal. M.G., E.W. and J.B. were supported by Israel

772 Science Foundation (\#1511/16); United States-Israel Binational Science Foundation

773 (\#2017325); Sagol Institute for Longevity and Estate of Emile Mimran . G.L. is an

774 incumbent of the Elias Sourasky Professorial Chair. This work was funded by the

775 project LISBOA-01-0145-FEDER-030627 co-funded by the Programa Operational

776 Regional de Lisboa (Lisboa 2020), through Portugal 2020 and the European Regional

777 Development Fund (FEDER), and by the FCT/MCTES through national funds

778 (PIDDAC).

\section{Authors contributions:}

780 ARN- Designed the research, performed experiments, analyzed data, wrote/revised 781 manuscript; MG - Designed the research, performed experiments, analyzed data, 782 wrote/revised manuscript; EW- Performed experiments; JB- Performed experiments; GP 783 and MT- Performed network analysis; SAMV- Performed statistical analysis; GL784 Designed the research, supervision, edited/revised manuscript; RO- Designed the 785 research, supervision, edited/revised manuscript 


\section{REFERENCES}

788

789

790

791

792

793

794

795

796

797

798

799

800

801

802

803

804

805

806

807

808

809

810

811

812

813

814

Anbalagan S, Blechman J, Gliksberg M, Gordon L, Rotkopf R, Dadosh T, Shimoni E, Levkowitz G (2019) Robo2 regulates synaptic oxytocin content by affecting actin dynamics. Elife 8:e45650.

Anbalagan S, Gordon L, Blechman J, Matsuoka RL, Rajamannar P, Wircer E, Biran J, Reuveny A, Leshkowitz D, Stainier DYR, Levkowitz G (2018) Pituicyte Cues Regulate the Development of Permeable Neuro-Vascular Interfaces. Dev Cell 47:711-726.e5.

Antinucci P, Folgueira M, Bianco IH (2019) Pretectal neurons control hunting behaviour. Elife 8:e48114.

Bartz JA, Zaki J, Bolger N, Ochsner KN (2011) Social effects of oxytocin in humans: context and person matter. Trends Cogn Sci 15:301-309.

Bates D, Mächler M, Bolker BM, Walker SC (2015) Fitting linear mixed-effects models using Ime4. J Stat Softw 67.

Biswal BB, Van Kylen J, Hyde JS (1997) Simultaneous assessment of flow and BOLD signals in resting-state functional connectivity maps. NMR Biomed 10:165-170.

Blechman J, Amir-Zilberstein L, Gutnick A, Ben-Dor S, Levkowitz G (2011) The metabolic regulator PGC-1 1 directly controls the expression of the hypothalamic neuropeptide oxytocin. J Neurosci 31:14835-14840.

Blechman J, Anbalagan S, Matthews GG, Levkowitz G (2018) Genome Editing Reveals Idiosyncrasy of CNGA2 Ion Channel-Directed Antibody Immunoreactivity Toward Oxytocin. Front Cell Dev Biol 6:117.

Borgatti SP, Everett MG, Johnson JC (2013) Analyzing Social Networks. Beverley Hills, CA: Sage Publications.

Borges AC, Pereira N, Franco M, Vale L, Pereira M, Cunha M V., Amaro A, Albuquerque T, Rebelo M (2016) Implementation of a Zebrafish Health Program in a Research Facility: A 4-Year Retrospective Study. Zebrafish 13:S115-S125.

Bowen MT, Neumann ID (2017) Rebalancing the Addicted Brain: Oxytocin Interference 
with the Neural Substrates of Addiction. Trends Neurosci 40:691-708.

816 Curado S, Anderson RM, Jungblut B, Mumm J, Schroeter E, Stainier DYR (2007)

817 Conditional targeted cell ablation in zebrafish: a new tool for regeneration studies.

818 Dev Dyn 236:1025-1035.

819 Curado S, Stainier DYR, Anderson RM (2008) Nitroreductase-mediated cell/tissue 820 ablation in zebrafish: A spatially and temporally controlled ablation method with applications in developmental and regeneration studies. Nat Protoc 3:948-954.

822 Davison JM, Akitake CM, Goll MG, Rhee JM, Gosse N, Baier H, Halpern ME, Leach SD, Parsons MJ (2007) Transactivation from Gal4-VP16 transgenic insertions for tissue-specific cell labeling and ablation in zebrafish. Dev Biol 304:811-824.

De Vico Fallani F, Latora V, Chavez M (2017) A Topological Criterion for Filtering Information in Complex Brain Networks. PLOS Comput Biol 13:e1005305.

827 Delignette-Muller ML, Dutang C (2015) fitdistrplus: An R package for fitting distributions. $828 \quad J$ Stat Softw 64:1-34.

829 Dölen G, Malenka RC (2014) The Emerging Role of Nucleus Accumbens Oxytocin in $830 \quad$ Social Cognition. Biol Psychiatry 76:354-355.

831 Donaldson ZR, Young LJ (2008) Oxytocin, vasopressin, and the neurogenetics of 832 sociality. Science (80- ) 322:900-904.

833 Dreosti E, Lopes G, Kampff AR, Wilson SW (2015) Development of social behavior in 834 young zebrafish. Front Neural Circuits 9:1-9.

835 Eickhoff SB, Müller V. (2015) Functional Connectivity. In: Brain Mapping (Toga AW, ed), 836 pp Pp. 187-201. Academic Press.

837 Engeszer RE, Barbiano LA DA, Ryan MJ, Parichy DM (2007) Timing and plasticity of 838 shoaling behaviour in the zebrafish, Danio rerio. Anim Behav 74:1269-1275.

839 Freeman SM, Inoue K, Smith AL, Goodman MM, Young LJ (2014a) The neuroanatomical distribution of oxytocin receptor binding and mRNA in the male rhesus macaque (Macaca mulatta). Psychoneuroendocrinology 45:128-141.

842 Freeman SM, Palumbo MC, Lawrence RH, Smith AL, Goodman MM, Bales KL (2018) 
Effect of age and autism spectrum disorder on oxytocin receptor density in the

845

\begin{abstract}
human basal forebrain and midbrain. Transl Psychiatry 8:257.
\end{abstract}
Freeman SM, Walum H, Inoue K, Smith AL, Goodman MM, Bales KL, Young LJ (2014b) Neuroanatomical distribution of oxytocin and vasopressin 1a receptors in the socially monogamous coppery titi monkey (Callicebus cupreus). Neuroscience 273:12-23.

Friston KJ (1994) Functional and effective connectivity in neuroimaging: A synthesis. Hum Brain Mapp 2:56-78.

Gamer M, Zurowski B, Büchel C (2010) Different amygdala subregions mediate valence-related and attentional effects of oxytocin in humans. Proc Natl Acad Sci U S A 107:9400-9405.

Goodson JL (2008) Nonapeptides and the evolutionary patterning of sociality. Prog Brain Res 170:3-15.

Grinevich V, Knobloch-Bollmann HS, Eliava M, Busnelli M, Chini B (2016) Assembling the Puzzle: Pathways of Oxytocin Signaling in the Brain. Biol Psychiatry 79:155164.

Grinevich V, Sophie Knobloch-Bollmann H, Eliava M, Busnelli M, Chini B (2015) Assembling the Puzzle: Pathways of Oxytocin Signaling in the Brain. Biol Psychiatry 79:155-164.

Grinevich V, Stoop R (2018) Interplay between Oxytocin and Sensory Systems in the Orchestration of Socio-Emotional Behaviors. Neuron 99:887-904.

Guastella AJ, Hickie IB (2016) Oxytocin Treatment, Circuitry, and Autism: A Critical Review of the Literature Placing Oxytocin into the Autism Context. Biol Psychiatry 79:234-242.

Guzmán YF, Tronson NC, Jovasevic V, Sato K, Guedea AL, Mizukami H, Nishimori K, Radulovic J (2013) Fear-enhancing effects of septal oxytocin receptors. Nat Neurosci 16:1185-1187.

Hammock EAD (2015) Developmental perspectives on oxytocin and vasopressin. 
Neuropsychopharmacology 40:24-42.

872 Heinrichs M, von Dawans B, Domes G (2009) Oxytocin, vasopressin, and human social 873 behavior. Front Neuroendocrinol 30:548-557.

874 Henrik Singmann, Ben Bolker, Jake Westfall FA and MSB-S (2020) afex: Analysis of $875 \quad$ Factorial Experiments. R package version 0.27-2.

876 Hörnberg H, Pérez-Garci E, Schreiner D, Hatstatt-Burklé L, Magara F, Baudouin S, Matter A, Nacro K, Pecho-Vrieseling E, Scheiffele P (2020) Rescue of oxytocin response and social behaviour in a mouse model of autism. Nature 584:252-256.

Hovey D, Zettergren A, Jonsson L, Melke J, Anckarsäter H, Lichtenstein P, Westberg L (2014) Associations between oxytocin-related genes and autistic-like traits. Soc Neurosci 9:378-386.

Hung LW, Neuner S, Polepalli JS, Beier KT, Wright M, Walsh JJ, Lewis EM, Luo L, Deisseroth K, Dölen G, Malenka RC (2017) Gating of social reward by oxytocin in the ventral tegmental area. Science (80- ) 357:1406-1411.

Insel TR, Shapiro LE (1992) Oxytocin receptor distribution reflects social organization in monogamous and polygamous voles. Proc Natl Acad Sci U S A 89:5981-5985.

Johnson Z V., Walum H, Xiao Y, Riefkohl PC, Young LJ (2017) Oxytocin receptors modulate a social salience neural network in male prairie voles. Horm Behav 87:16-24.

Jurek B, Neumann ID (2018) The oxytocin receptor: From intracellular signaling to behavior. Physiol Rev 98:1805-1908.

Keebaugh AC, Young LJ (2011) Increasing oxytocin receptor expression in the nucleus accumbens of pre-pubertal female prairie voles enhances alloparental responsiveness and partner preference formation as adults. Horm Behav 60:498504.

Kizil C, Kaslin J, Kroehne V, Brand M (2012) Adult neurogenesis and brain regeneration

898 Knight ZA, Tan K, Birsoy K, Schmidt S, Garrison JL, Wysocki RW, Emiliano A, Ekstrand 
MI, Friedman JM (2012) Molecular profiling of activated neurons by phosphorylated ribosome capture. Cell 151:1126-1137.

901 Knobloch HS, Grinevich V (2014) Evolution of oxytocin pathways in the brain of 902 vertebrates. Front Behav Neurosci 8.

903 Krauzlis RJ, Liston D, Carello CD (2004) Target selection and the superior colliculus: Goals, choices and hypotheses. In: Vision Research, pp 1445-1451.

Kret ME, De Dreu CKW (2017) Pupil-mimicry conditions trust in partners: Moderation by oxytocin and group membership. Proc R Soc B Biol Sci 284.

Leknes S, Wessberg J, Ellingsen D-M, Chelnokova O, Olausson H, Laeng B (2013) Oxytocin enhances pupil dilation and sensitivity to 'hidden' emotional expressions. Soc Cogn Affect Neurosci 8:741-749.

Lenth R (2020) emmeans: Estimated Marginal Means, aka Least-Squares Means. R package version 1.4.7.

Lorenzi E, Mayer U, Rosa-Salva O, Vallortigara G (2017) Dynamic features of animate motion activate septal and preoptic areas in visually naïve chicks (Gallus gallus). Neuroscience 354:54-68.

Love TM (2014) Oxytocin, motivation and the role of dopamine. Pharmacol Biochem Behav 119:49-60.

Ludwig M, Leng G (2006) Dendritic peptide release and peptide-dependent behaviours. neuropeptide oxytocin facilitates pro-social behavior and prevents social avoidance

Ma PM (1994a) Catecholaminergic systems in the zebrafish. II. Projection pathways in rats and mice. Neuropsychopharmacology 36:2159-2168.

924 Ma PM (1994b) Catecholaminergic systems in the zebrafish. I. Number, morphology, 925 and histochemical characteristics of neurons in the locus coeruleus. J Comp Neurol $926 \quad 344: 242-255$. 
Ma PM (1997) Catecholaminergic systems in the zebrafish. III. organization and projection pattern of medullary dopaminergic and noradrenergic neurons. J Comp Neurol 381:411-427.

Machluf Y, Levkowitz G (2011) Visualization of mRNA expression in the zebrafish embryo. Methods Mol Biol 714:83-102.

Makagon MM, McCowan B, Mench JA (2012) How can social network analysis contribute to social behavior research in applied ethology? Appl Anim Behav Sci 138:152-161.

Martin LJ, Spicer DM, Lewis MH, Gluck JP, Cork LC (1991) Social deprivation of infant rhesus monkeys alters the chemoarchitecture of the brain: I. Subcortical regions. J Neurosci 11:3344-3358.

Menon R, Grund T, Zoicas I, Althammer F, Fiedler D, Biermeier V, Bosch OJ, Hiraoka Y, Nishimori K, Eliava M, Grinevich V, Neumann ID (2018) Oxytocin Signaling in the Lateral Septum Prevents Social Fear during Lactation. Curr Biol 28:10661078.e6.

Meyer MP, Smith SJ (2006) Evidence from in vivo imaging that synaptogenesis guides

Miller T V., Caldwell HK (2015) Oxytocin during development: Possible organizational effects on behavior. Front Endocrinol (Lausanne) 6:76.

947 Morales M, Margolis EB (2017) Ventral tegmental area: Cellular heterogeneity, 948 connectivity and behaviour. Nat Rev Neurosci 18:73-85.

949 Noonan LR, Continella G, Pedersen CA (1989) Neonatal administration of oxytocin 950 increases novelty-induced grooming in the adult rat. Pharmacol Biochem Behav 33:555-558.

952 Nunes AR, Carreira L, Anbalagan S, Blechman J, Levkowitz G, Oliveira RF (2020)

953 Perceptual mechanisms of social affiliation in zebrafish. Sci Rep 10:1-14.

954 Nunes AR, Ruhl N, Winberg S, Oliveira RF (2017) Social phenotypes in zebrafish. In: 
The Rights and Wrongs of Zebrafish: Behavioral Phenotyping of Zebrafish, pp 95130. Springer International Publishing.

957 O'Connell LA, Hofmann HA (2011) The Vertebrate mesolimbic reward system and social behavior network: A comparative synthesis. J Comp Neurol 519:3599-3639.

O'Connell LA, Hofmann HA (2012) Evolution of a vertebrate social decision-making network. Science (80- ) 336:1154-1157.

Panula P, Chen YC, Priyadarshini M, Kudo H, Semenova S, Sundvik M, Sallinen V (2010) The comparative neuroanatomy and neurochemistry of zebrafish CNS systems of relevance to human neuropsychiatric diseases. Neurobiol Dis 40:46-57.

Park MJ, Seo BA, Lee B, Shin HS, Kang MG (2018) Stress-induced changes in social dominance are scaled by AMPA-type glutamate receptor phosphorylation in the medial prefrontal cortex. Sci Rep 8:15008.

Parker MO, Brock AJ, Walton RT, Brennan CH (2013) The role of zebrafish (Danio

Peixoto TP (2021) Revealing Consensus and Dissensus between Network Partitions. Phys Rev X 11:021003.

972 Phoenix CH, Goy RW, Geral AA, Young WC (1959) Organizing action of prenatally administered testosterone propionate on the tissues mediating mating behavior in the female guinea pig. Endocrinology 65:369-382.

975 Pobbe RLH, Pearson BL, Defensor EB, Bolivar VJ, Young WS, Lee H-J, Blanchard DC, Blanchard RJ, Blanchard RJ (2012) Oxytocin receptor knockout mice display deficits in the expression of autism-related behaviors. Horm Behav 61:436-444.

978 R_core_Team (2013) R: A language and environment for statistical computing.

979 Rajamani KT, Wagner S, Grinevich V, Harony-Nicolas H (2018) Oxytocin as a 980 Modulator of Synaptic Plasticity: Implications for Neurodevelopmental Disorders. $981 \quad$ Front Synaptic Neurosci 10:17.

982 Ribeiro D, Nunes AR, Gliksberg M, Anbalagan S, Levkowitz G, Oliveira RF (2020a) 
Oxytocin receptor signalling modulates novelty recognition but not social preference in zebrafish. J Neuroendocrinol 32:e12834.

Ribeiro D, Nunes AR, Teles M, Anbalagan S, Blechman J, Levkowitz G, Oliveira RF (2020b) Genetic variation in the social environment affects behavioral phenotypes of oxytocin receptor mutants in zebrafish. Elife 9:e56973.

Rink E, Wullimann MF (2001) The teleostean (zebrafish) dopaminergic system ascending to the subpallium (striatum) is located in the basal diencephalon (posterior tuberculum). Brain Res 889:316-330.

Rink E, Wullimann MF (2002) Connections of the ventral telencephalon and tyrosine hydroxylase distribution in the zebrafish brain (Danio rerio) lead to identification of an ascending dopaminergic system in a teleost. In: Brain Research Bulletin, pp 385-387.

Robinson KJ, Bosch OJ, Levkowitz G, Busch KE, Jarman AP, Ludwig M (2019) Social

Saito D, Komatsuda M, Urano A (2004) Functional organization of preoptic vasotocin and isotocin neurons in the brain of rainbow trout: Central and neurohypophysial projections of single neurons. Neuroscience 124:973-984. MPTP and MPP+ target specific aminergic cell populations in larval zebrafish. J Neurochem 108:719-731.

Schindelin J, Arganda-Carreras I, Frise E, Kaynig V, Longair M, Pietzsch T, Preibisch S,

Sawchenko PE, Swanson LW (1982) Immunohistochemical identification of neurons in the paraventricular nucleus of the hypothalamus that project to the medulla or to the spinal cord in the rat. J Comp Neurol 205:260-272.

Rueden C, Saalfeld S, Schmid B, Tinevez JY, White DJ, Hartenstein V, Eliceiri K, Tomancak P, Cardona A (2012) Fiji: An open-source platform for biological-image analysis. Nat Methods 9:676-682. 
oxytocin binding sites in the brain and upper spinal cord of the common marmoset. Neurosci Lett 461:217-222.

1014 Shamay-Tsoory SG, Abu-Akel A (2016) The Social Salience Hypothesis of Oxytocin. 1015 Biol Psychiatry 79:194-202.

1016 Son SJ, Filosa JA, Potapenko ES, Biancardi VC, Zheng H, Patel KP, Tobin VA, Ludwig M, Stern JE (2013) Dendritic peptide release mediates interpopulation crosstalk between neurosecretory and preautonomic networks. Neuron 78:1036-1049.

Stednitz SJ, McDermott EM, Ncube D, Tallafuss A, Eisen JS, Washbourne P (2018) Forebrain Control of Behaviorally Driven Social Orienting in Zebrafish. Curr Biol 28:2445-2451.e3.

Tay TL, Ronneberger O, Ryu S, Nitschke R, Driever W (2011) Comprehensive catecholaminergic projectome analysis reveals single-neuron integration of zebrafish ascending and descending dopaminergic systems. Nat Commun 2:171.

Teles MC, Almeida O, Lopes JSJSJS, Oliveira RF (2015) Social interactions elicit rapid 1026 shifts in functional connectivity in the social decision-making network of zebrafish.

Traag VA, Waltman L, van Eck NJ (2019) From Louvain to Leiden: guaranteeing wellconnected communities. Sci Rep 9:1-12.

Van den Dungen HM, Buijs RM, Pool CW, Terlou M (1982) The distribution of vasotocin and isotocin in the brain of the rainbow trout. J Comp Neurol 212:146-157.

Vega-Quiroga I, Yarur HE, Gysling K (2018) Lateral septum stimulation disinhibits dopaminergic neurons in the antero-ventral region of the ventral tegmental area: Role of GABA-A alpha 1 receptors. Neuropharmacology 128:76-85.

von Trotha JW, Vernier P, Bally-Cuif L (2014) Emotions and motivated behavior converge on an amygdala-like structure in the zebrafish. Eur J Neurosci 40:33023315.

Wee CL, Nikitchenko M, Wang WC, Luks-Morgan SJ, Song E, Gagnon JA, Randlett O, Bianco IH, Lacoste AMB, Glushenkova E, Barrios JP, Schier AF, Kunes S, Engert 
F, Douglass AD (2019) Zebrafish oxytocin neurons drive nocifensive behavior via brainstem premotor targets. Nat Neurosci 22:1477-1492.

1042 Wircer E, Blechman J, Borodovsky N, Tsoory M, Nunes AR, Oliveira RF, Levkowitz G 1043 (2017) Homeodomain protein otp affects developmental neuropeptide switching in 1044 oxytocin neurons associated with a long-term effect on social behavior. Elife $1045 \quad 6: \mathrm{e} 22170$.

1046 Wullimann MF, Mueller T (2004) Teleostean and mammalian forebrains contrasted:

1047 Evidence from genes to behavior. J Comp Neurol 475:143-162.

1048 Wullimann MF, Rupp B, Reichert H (1996) Neuroanatomy of the Zebrafish Brain. 1049 Birkhäuser Basel.

1050 Zhang B, Qiu L, Xiao W, Ni H, Chen L, Wang F, Mai W, Wu J, Bao A, Hu H, Gong H, 1051 Duan S, Li A, Gao Z (2021) Reconstruction of the Hypothalamo-Neurohypophysial 1052 System and Functional Dissection of Magnocellular Oxytocin Neurons in the Brain. Neuron 109:331-346.e7. 


\section{A shoal visible B shoal preference $\quad$ C 1.0}

bioRxiv preprint doi: https://doi.org/10.1101/2020.10.08.330993; this version posted April 29, 2021. The copyright holder for this predrint (\#hich

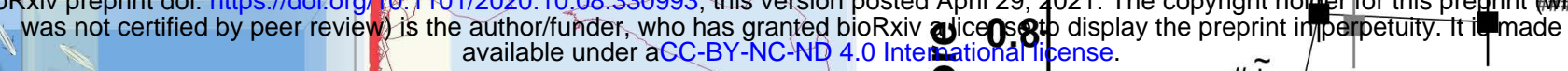

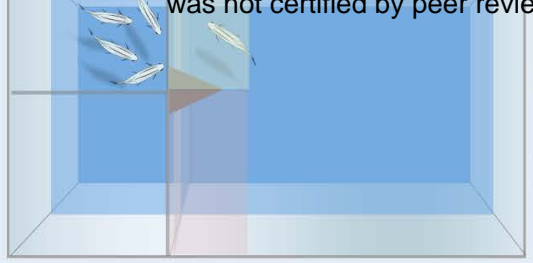

Shoal not visible

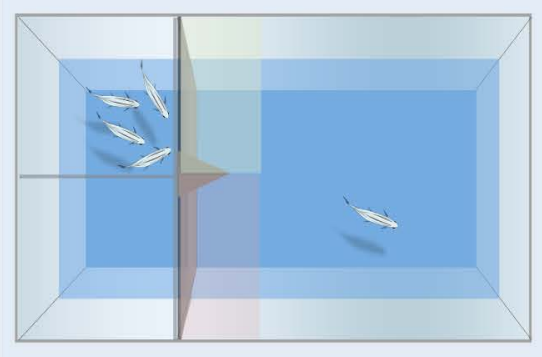

\section{No preference}

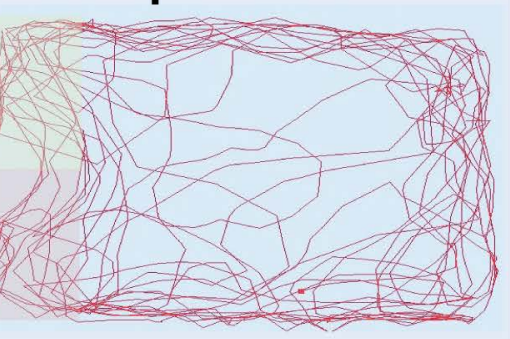

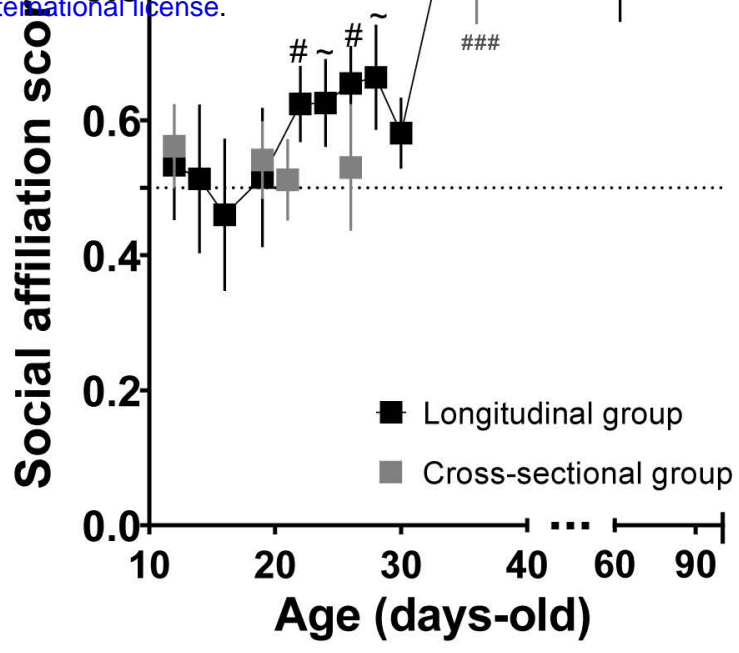

\section{\begin{tabular}{l|l|l|}
\hline Gal4 $\times$ UAS & NTR-mCherry \\
\hline
\end{tabular}}

E

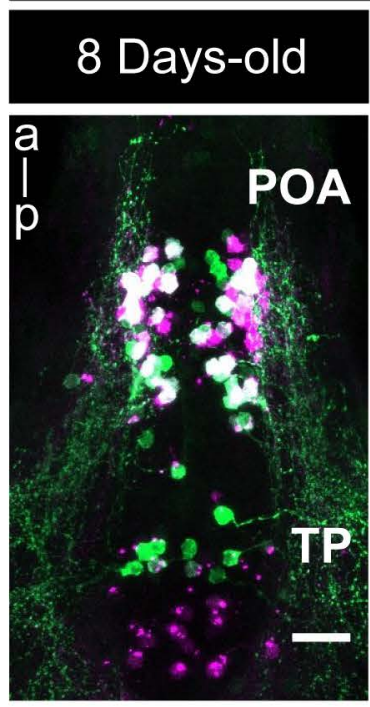

\section{oxt:EGFP, oxt:Gal4;UAS:NTR-mCherry}

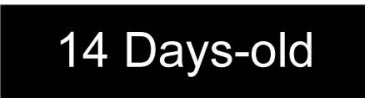

24 Days-old

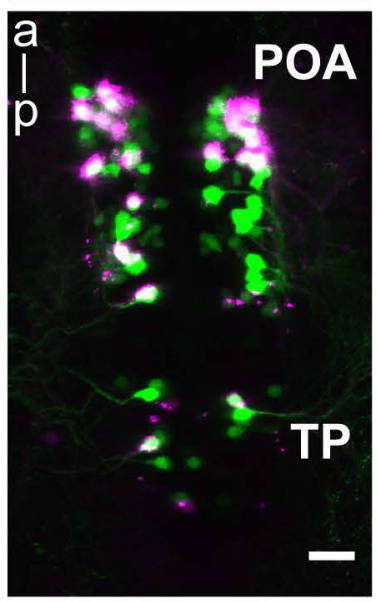

90 Days-old

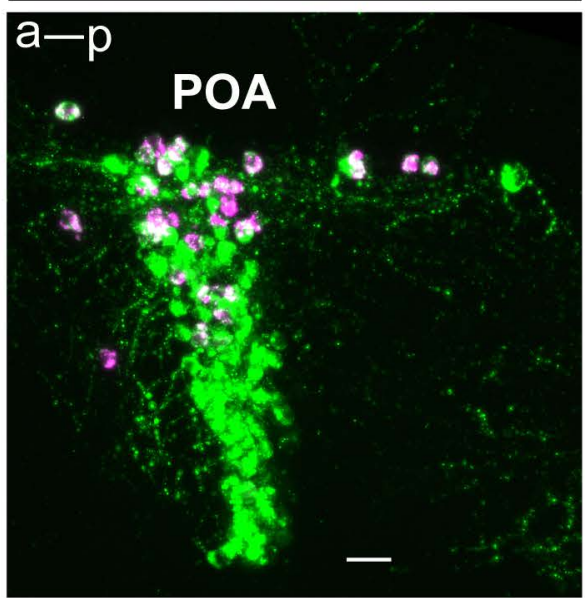




\section{Legends to Figures}

\section{Figure 1. Behavioral and genetic tools to study the role of oxytocinergic neurons} in adult social affiliation. (A,B) Schematic representation of the visual-mediated shoal preference behavioral test setup. (A) When a mixed-sex shoal stimulus is visible, focal fish spends most of the time near the shoal (top). When the shoal is not visible (bottom), the focal fish explores the entire arena equally. (B) Representative tracking of the focal fish behavior. (C) Ontogeny of social affiliation in zebrafish. Visual preference to associate with a shoal of conspecific fish (social affiliation score $>0.5$ ) emerges after the third week of life. Social affiliation score $=\% \mathrm{~T}_{\text {shoal }}\left(\% \mathrm{~T}_{\text {shoal }}+\% \mathrm{~T}_{\text {non-shoal }}\right)$. \# Hash indicates a significant preference towards conspecifics, determined by a one-sample $t$-test with a hypothesized value of 0.5 (chance level). Fish were tested either repeatedly throughout development (black squares, longitudinal group) or only once at certain developmental time points (grey squares, cross-sectional group). (D) Spatio-temporal control of oxytocinspecific transgene expression. oxt:Gal4 drives the expression of nitroreductase tagged with mCherry fluorescent protein (NTR-mCherry) in oxytocinergic neurons. (E) Tg(oxt:Gal4;UAS:NTR-mCherry) characterization: NTR-mCherry+ cells co-localized with a subpopulation of oxt:eGFP neurons, at all developmental stages studied (8, 14 and 24 days-old: whole-mount larvae confocal z-stack image, dorsal view, anterior to top; 90 days-old, close-up of the preoptic area (POA), sagittal brain slice, confocal z-stack image, anterior to left).

Scale: $20 \mu \mathrm{m}$. TP, Posterior Tuberculum; POA, Preoptic area. Data presented as mean \pm SEM.

$\# p<0.05, \# \# p<0.01, \# \#$ p $<0.001$, \#\#\# p<0.0001 (black \# relates to the longitudinal group, grey \# relates to the cross-sectional group). 

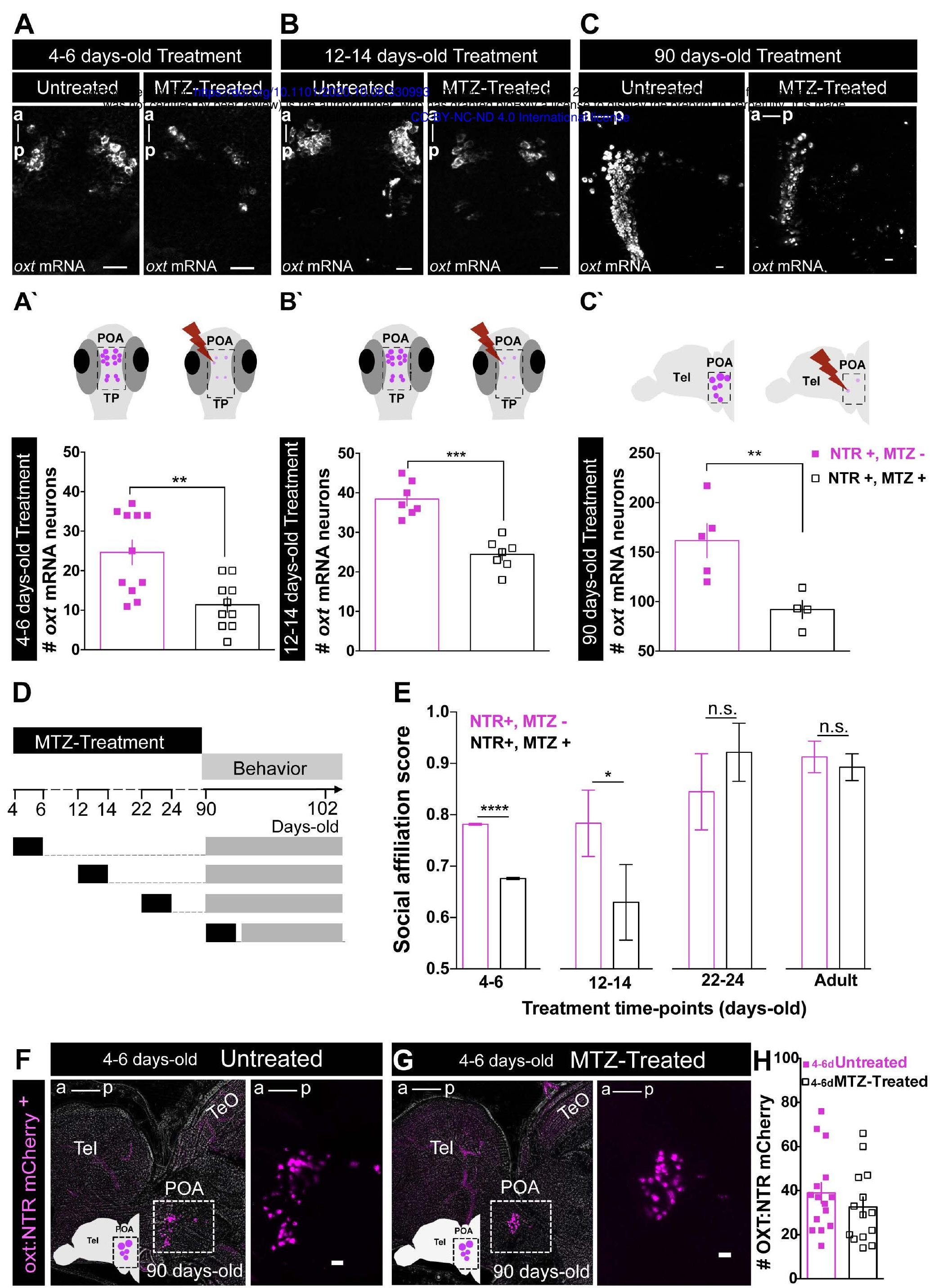
Figure 2. Contrasting organizational vs activational effects of oxytocin neurons in adult social affiliation. (A-C) Representative examples of the effects of metronidazole (MTZ)- induced ablation on endogenous OXT as detected by in situ hybridization, at different treatment time-points. (A) 4-6 days-old treatment and (B) 12-14 days-old treatment: whole-mount larvae confocal maximum intensity z-stack image, dorsal view, anterior to top; (C) 90 days-old treatment: sagittal brain slice, confocal maximum intensity z-stack image, anterior to left. $\left(A^{\prime}-C^{\prime}\right)$ Quantification of the number of cells expressing OXT mRNA in untreated fish or following MTZ treatment at: ( $\left.\mathrm{A}^{\prime}\right)$ 4-6 days-old treatment, (B') 12-14 days-old treatment and ( $C^{\prime}$ ) 90 days-old treatment. (D) Experimental timeline: fish were MTZ-treated at different developmental stages, allowed to grow until adulthood and then tested for social affiliation. (E) MTZ treatments during the first two weeks of life affect adult social affiliation. Social affiliation score $=\% \mathrm{~T}_{\text {shoal }} /\left(\% \mathrm{~T}_{\text {shoal }}+\% \mathrm{~T}_{\text {non-shoal }}\right)$. Eight independent cohorts were treated at 4-6 days-old, and only one cohort at later time points. Therefore, in order to obtain comparable sample sizes among the different age-treatment groups and a more representative sample, the data for 4-6 days-old MTZ treatment shown in this graph were generated by a Monte Carlo simulation with 1000 iterations of samples of 15 individuals of each group (4-6 days-old MTZ-treated vs. untreated; see Statistical analysis subsection for details). One-tailed $p$-values were used because there was an a priori directional hypothesis that MTZ-treatment would ablate OXT neurons in fish expressing the NTR transgene and induce a decrease in social affiliation behavior. (F-H) OXT neurons ablated at 4-6 days-old were recovered by adulthood. (F) Representative example of NTR-mCherry-expressing OXT neurons in untreated adult fish. (G) Representative example of NTR-mCherry-expressing OXT neurons in adult fish treated with MTZ at 4-6 days-old. Images in $(F, G)$ are maximum intensity confocal zstacks, sagittal slices, anterior to left. $(H)$ Quantification of the number of NTR-mCherry cells in untreated (full squares) and 4-6 days-old MTZ-treated adult fish (empty squares). Scale: $20 \mu \mathrm{m}$. Data presented as mean \pm SEM. Full squares: untreated fish (NTR+, MTZ); Open squares: MTZ-treated fish (NTR+, MTZ+).

${ }^{*} p<0.05,{ }^{* *} p<0.01,{ }^{* * *} p<0.001$. ${ }^{* * * *} p<0.0001$. 
was not certified by peer review) is the author/funder, who has granted bioRxiv a license to display the preprint in perpetuity. It is made

A

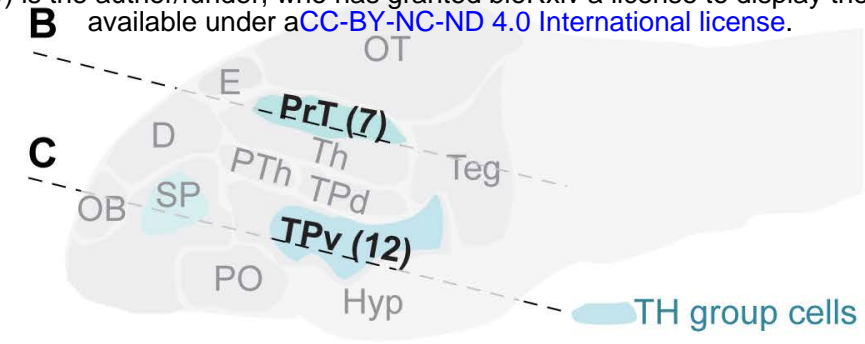

B
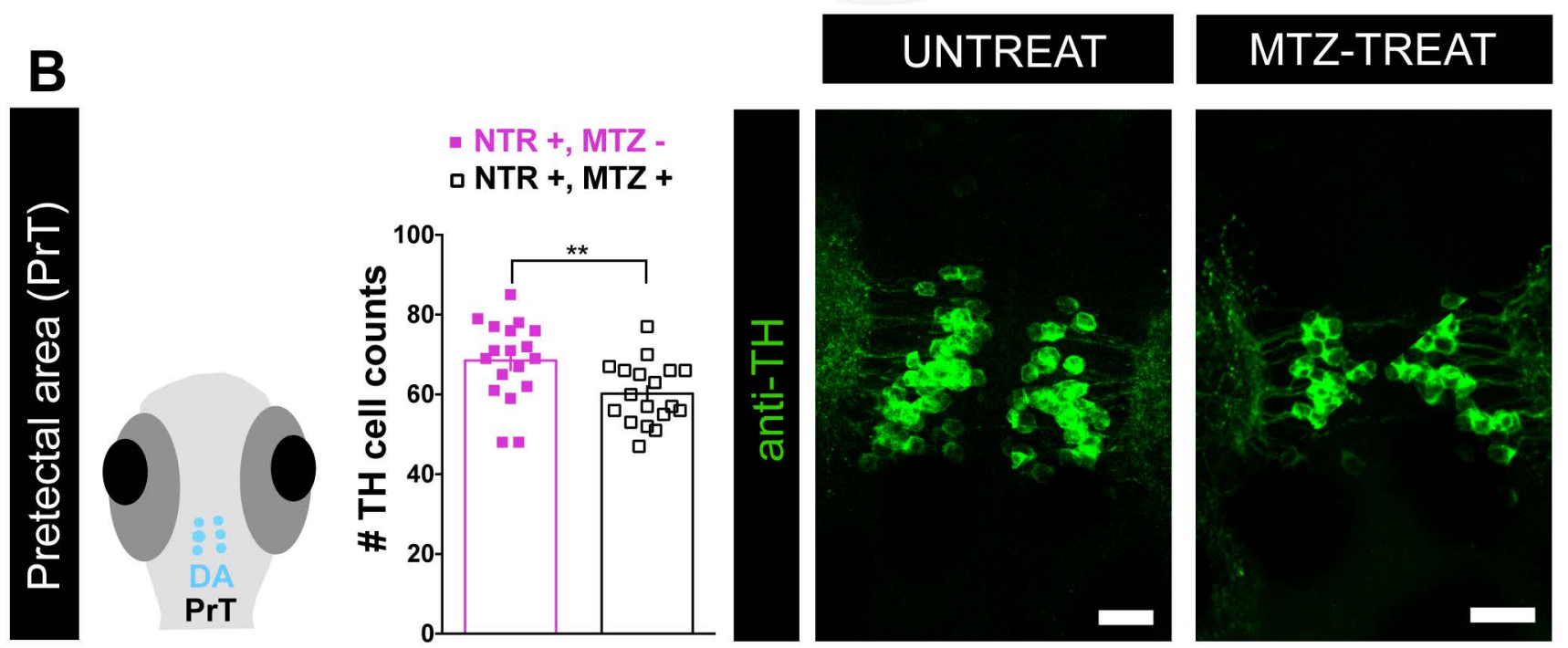

C
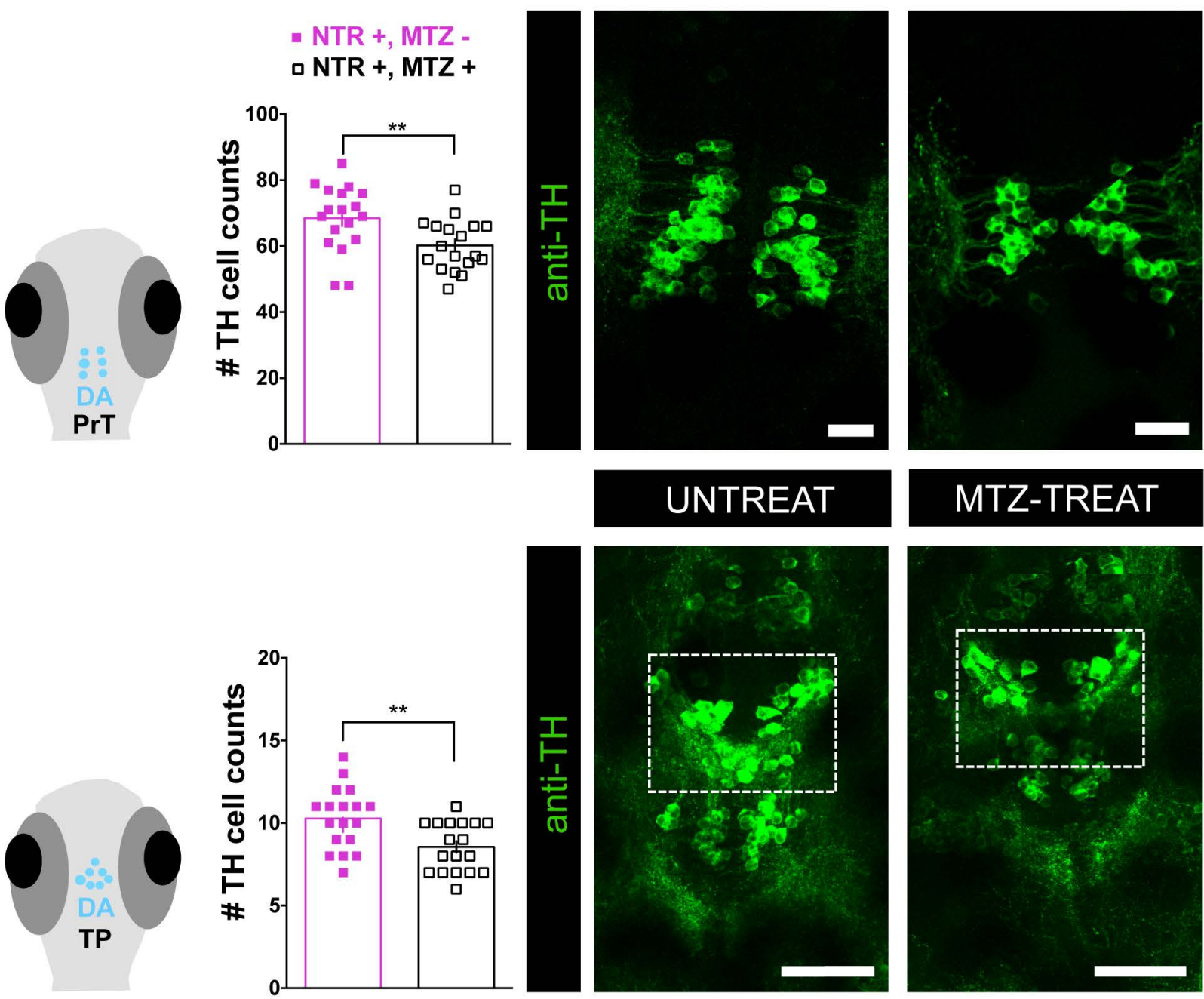

\section{UNTREAT}
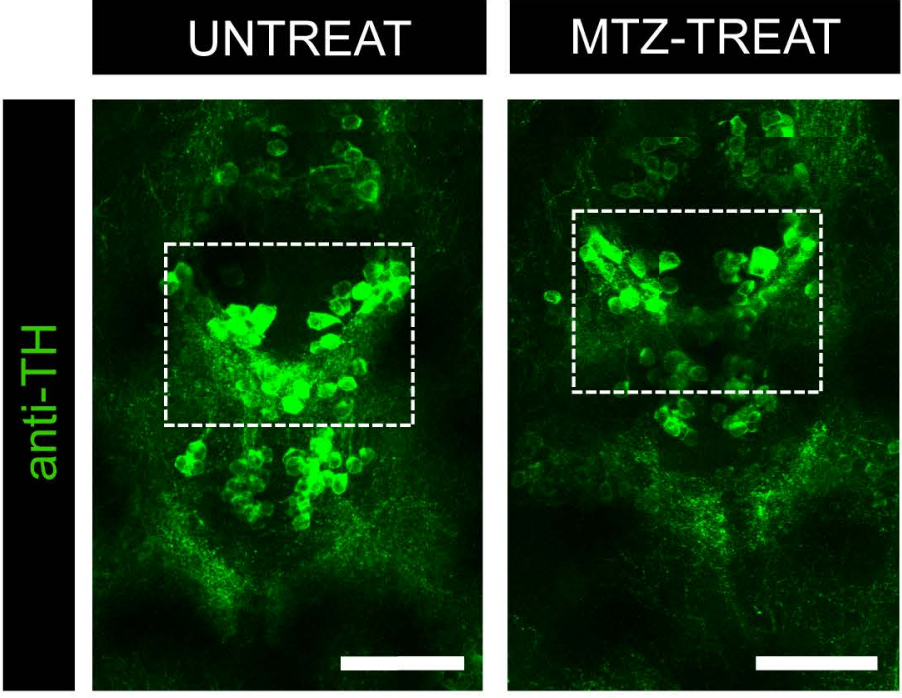
bioRxiv preprint doi: https://doi.org/10.1101/2020.10.08.330993; this version posted April 29, 2021. Theddopyright Koldel'tor this preprint (which was not certified by peer review) is the author/funder, who has granted bioRxiv a license to disspay the preprint in (perpetuity. It is made

$$
\begin{aligned}
& 3 \text { months-old } \\
& \mathrm{OB}
\end{aligned}
$$

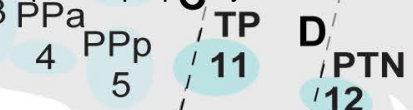

B

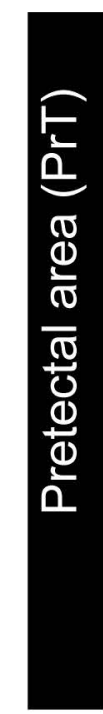

C

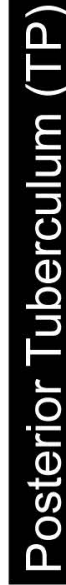

E

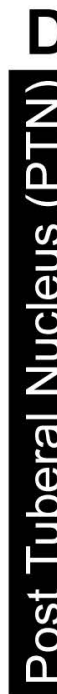

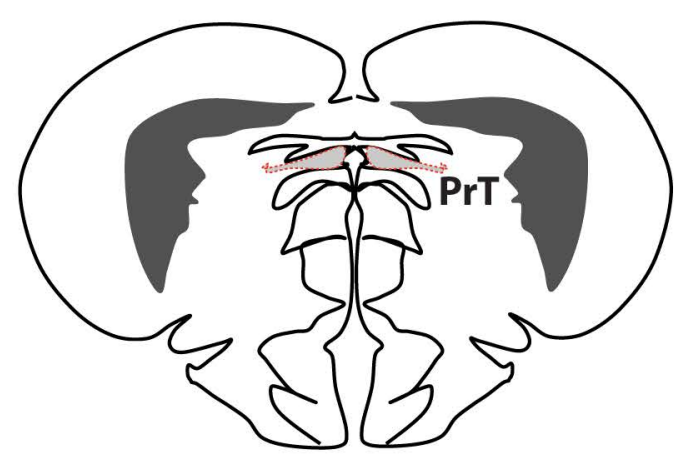
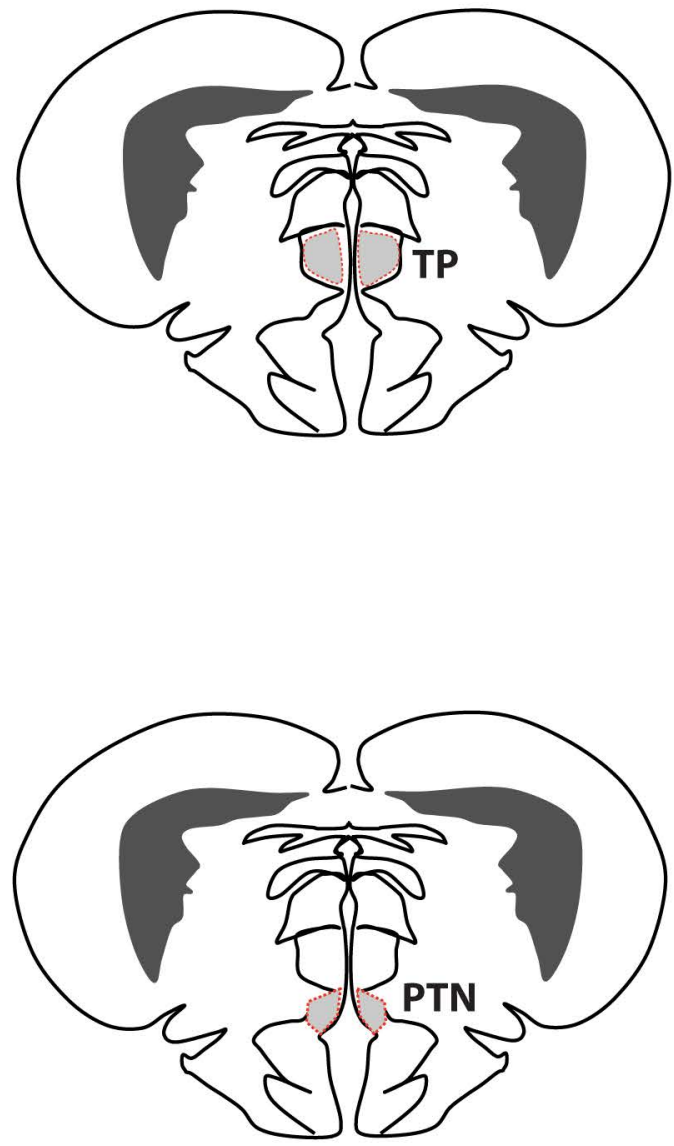
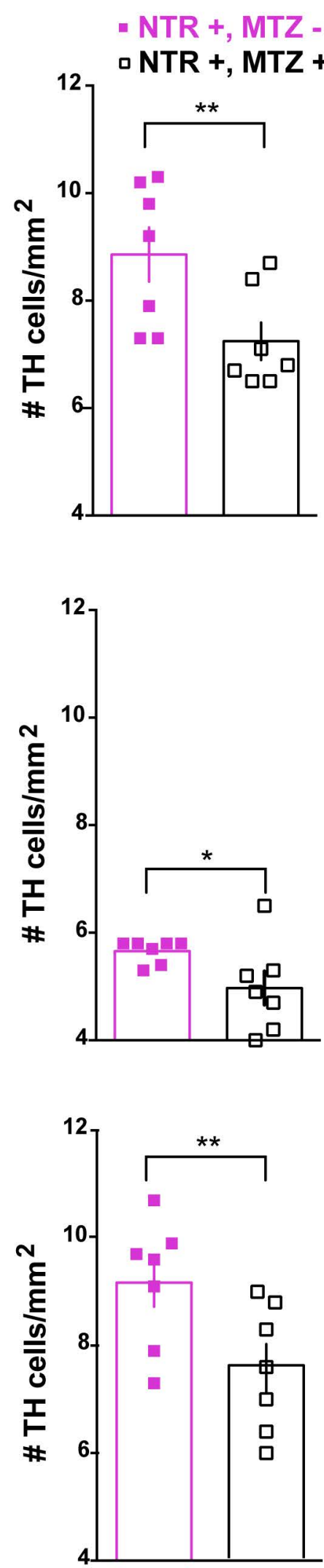

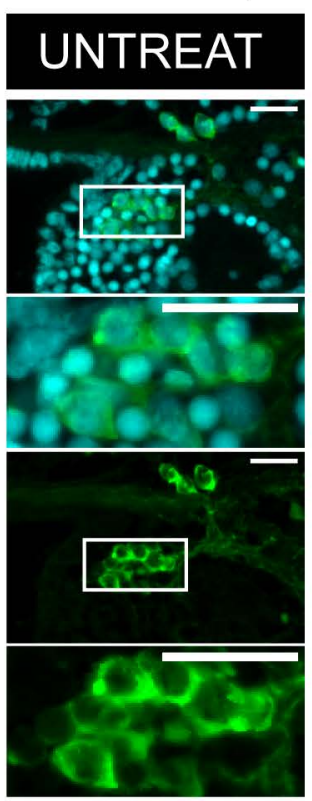

\section{MTZ-TREAT}
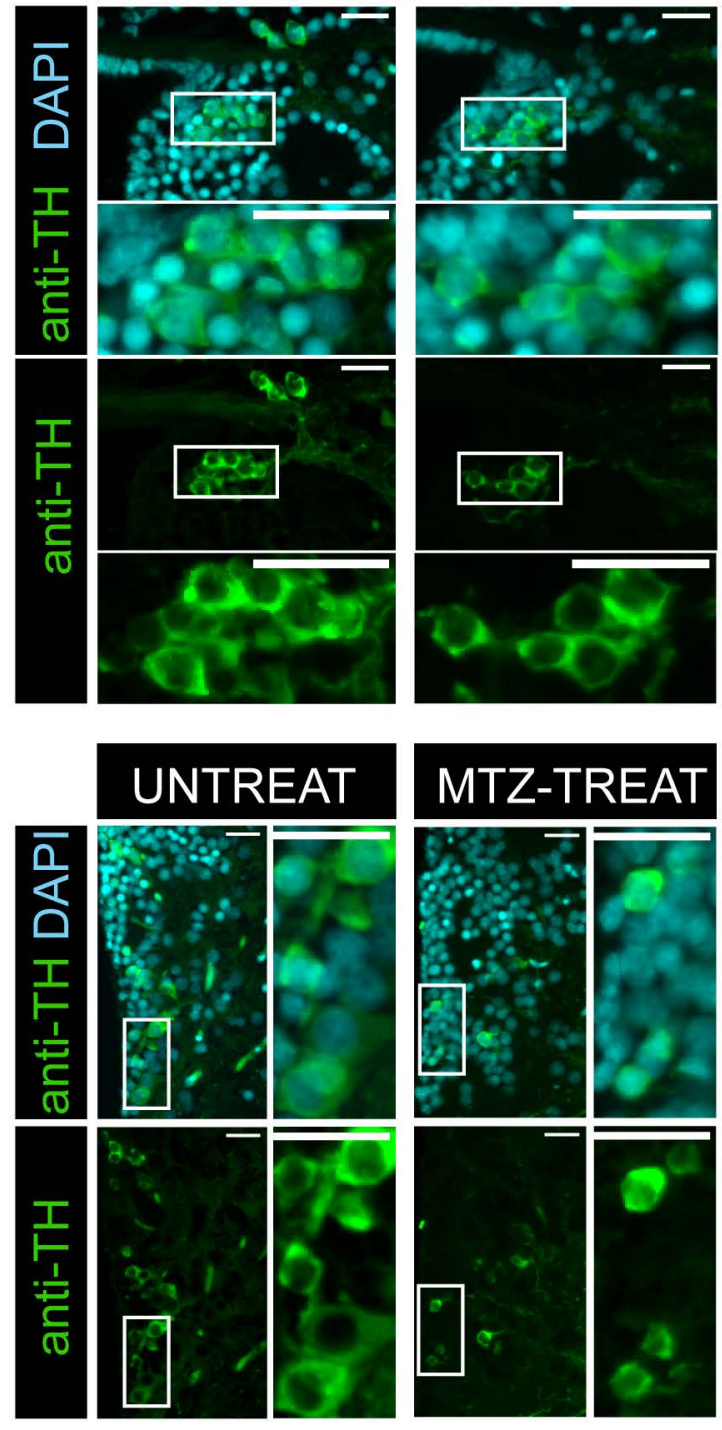

\section{UNTREAT}

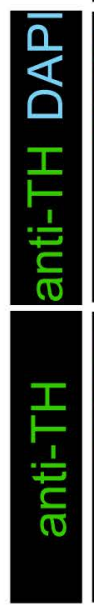

\section{MTZ-TREAT}

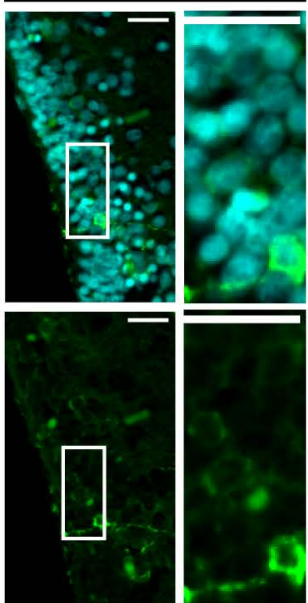


Figure 3. Early-life OXT ablation affects the dopaminergic system in early development. (A) Top: Schematic sagittal section through a zebrafish larva brain showing the three dopaminergic clusters that were analyzed in this study, and highlighting two key dopaminergic brain areas that were affected by OXT neuronal ablation at 4-6 days-old, namely the pretectum (PrT) and posterior tuberculum (TP). Schematic was adapted from zebrafishucl.org/forebrain-regions/posterior-tuberculum. (B) Quantification of total pretectum (PrT) TH cell counts in untreated (NTR+, MTZ-) vs 4-6 days-old MTZtreated (NTR+, MTZ+) larvae and respective representative images. Scale: $20 \mu \mathrm{m}$. (C) Quantification of total Posterior Tuberculum (TP) TH cell counts in untreated (NTR+, MTZ) vs 4-6 days-old MTZ-treated (NTR+, MTZ+) larvae and respective representative images. Scale: $50 \mu \mathrm{m}$

. Data presented as mean \pm SEM. Full squares: untreated fish (NTR+, MTZ); Open squares: MTZ-treated fish (NTR+, MTZ+).

${ }^{*} p<0.05,{ }^{* *} p<0.01,{ }^{* * *} p<0.001 .{ }^{* * * *} p<0.0001$.

$D$, dorsal telencephalon; $E$, epiphysis; $O B$, olfactory bulb; $O T$, optic tectum; $P O$, preoptic area; $P r T$, pretectum; $T P d$, posterior tuberculum dorsal part; PTh, prethalamus; $T P V$, posterior tuberculum ventral part; Teg, tegmentum; Th, thalamus; $S P$, subpallium (includes $\mathrm{V} v, \mathrm{Vd}$ and $\mathrm{Vs}$ in adult); Hyp, hypothalamus; NTR, nitroreductase; MTZ, metronidazole.

Figure 4. Early-life OXT ablation affects the dopaminergic system in adulthood. (A) Schematic sagittal section through a zebrafish adult brain, showing the eight dopaminergic clusters analyzed in this study, and highlighting the areas that were affected by OXT neuronal ablation at 4-6 days-old: Pretectal area (PrT), Posterior Tuberculum (TP) and Posterior Tuberal Nucleus (PTN). Dopaminergic groups are named according to (Panula et al., 2010; Parker et al., 2013). (B-D) Quantification of TH cell density (cells $/ \mathrm{mm}^{2}$; mean of five sampled slices for each area, see Methods section for details) in brains of adult fish untreated (NTR+, MTZ-) or MTZ-treated at 4-6 days-old (NTR+, $\mathrm{MTZ}+$ ) and respective representative images in: (B) Pretectal area (PrT), (C) Posterior Tuberculum (TP) and (D) Posterior Tuberal Nucleus (PTN) 
Scale: $20 \mu \mathrm{m}$. Data presented as mean \pm SEM. Full squares: untreated fish (NTR+, MTZ); Open squares: MTZ-treated fish (NTR+, MTZ+).

${ }^{*} p<0.05,{ }^{* *} p<0.01,{ }^{* * *} p<0.001 .{ }^{* * * *} p<0.0001$.

$D$, dorsal telencephalon; $O B$, olfactory bulb; $O T$, optic tectum; $\operatorname{PrT}$, pretectum; TP, posterior tuberculum; $S P$, subpallium (includes $\mathrm{Vv}, \mathrm{Vd}$ and $\mathrm{Vs}$ ); $P p a$, anterior parvocellular preoptic nucleus; $V M$, ventromedial thalamic nucleus; VI, ventrolateral thalamic nucleus; PPp, posterior parvocellular preoptic nucleus; PTN, posterior tuberal nucleus; Hyp, hypothalamus; NTR, nitroreductase; MTZ, metronidazole. 
C

bioRxiv preprimt doi: https://doi.org/10.1101/2020.10.08.330993,-this version posted April 29, 2021. The copyright holder for this preprint (w oxtw36.50 drtiteshypeer review) is the author/funder, who has granted bioRxiv a license to display the preprint in perpetuity. It is made

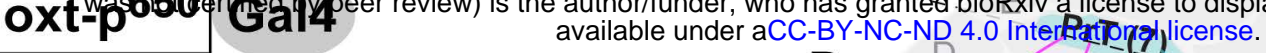

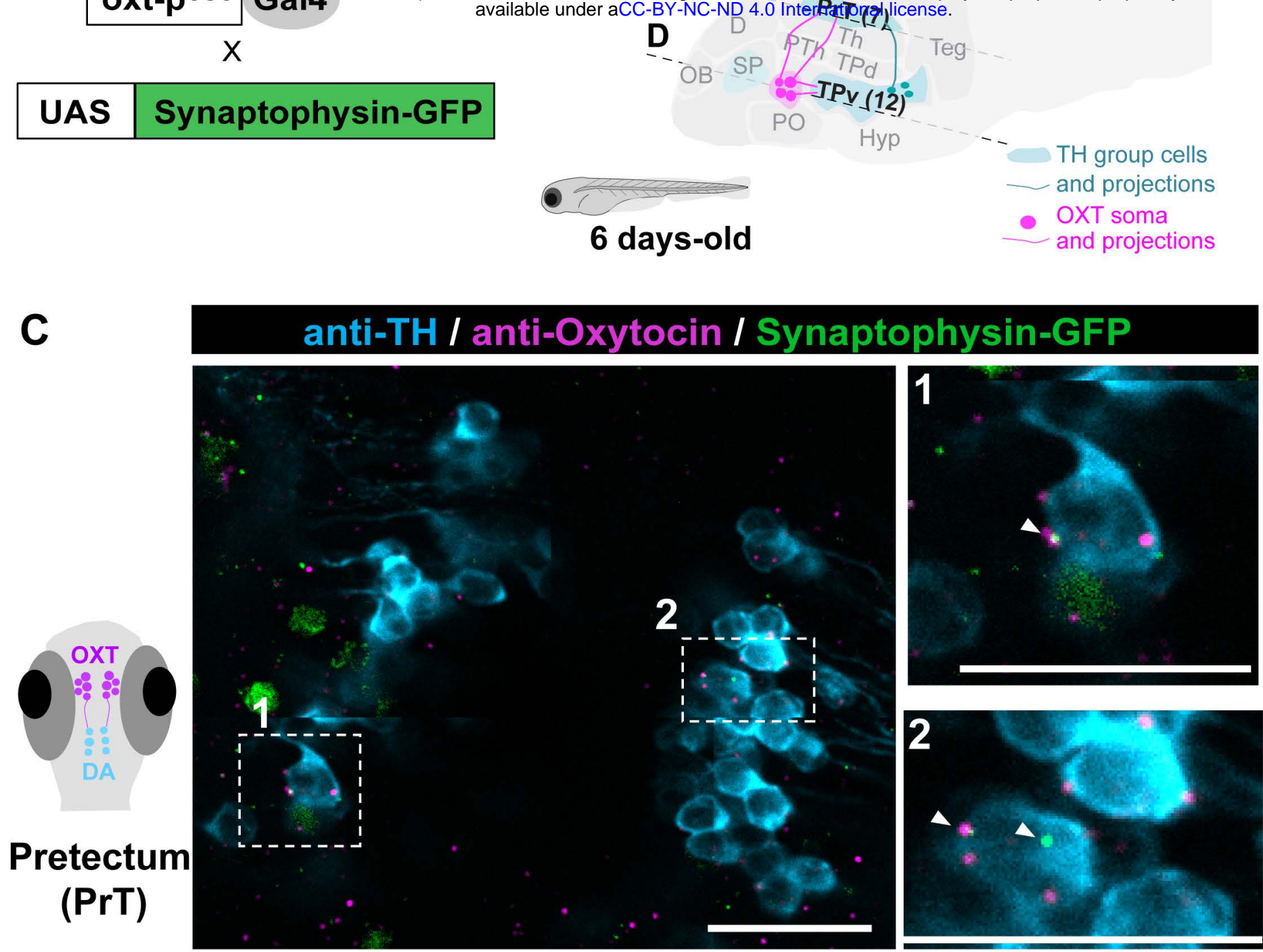

D

anti-TH / anti-Oxytocin / Synaptophysin-GFP
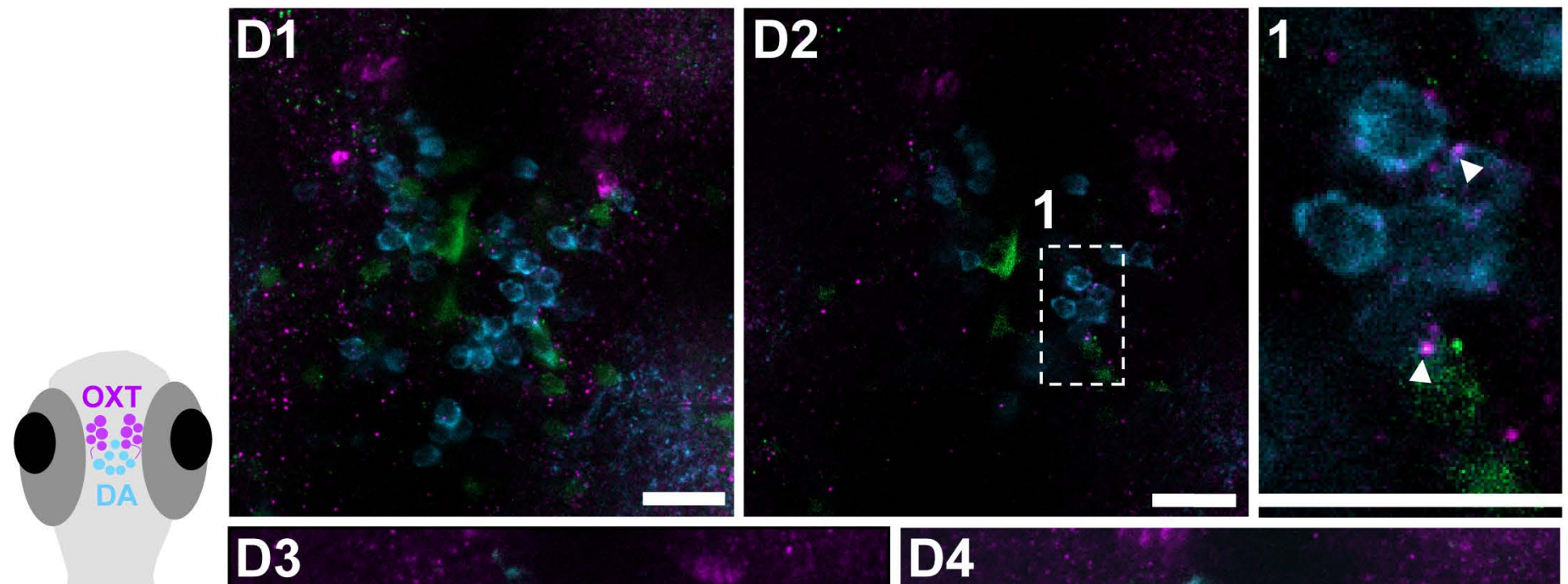

\section{Posterior} tuberculum (TP)

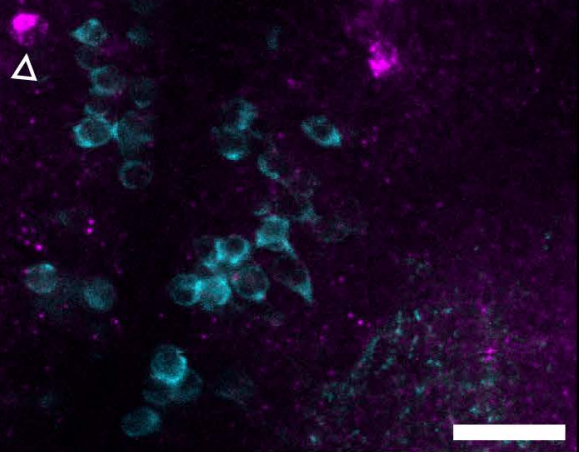


Figure 5. OXT neurons project to pretectal and posterior tuberculum dopaminergic clusters. (A) OXT neuron-specific labeling of synapses: oxt:Gal4 drives the expression of the EGFP-fused synaptic vesicle marker synaptophysin-B in oxytocinergic synapses. (B) Schematic of a sagittal larva brain highlighting OXT neuronal projections (in magenta) to pretectal and posterior tuberculum dopaminergic clusters (in blue). (C-D) Representative example showing that the synaptic marker line synaptophysin-GFP (green), driven under the OXT promoter, reveals bona fide OXT synapses containing the OXT peptide (anti-OXT, magenta) on TH positive cells (cyan) in both (C) pretectum (scale: $10 \mu \mathrm{m}$ ) and (D1-D2) posterior tuberculum clusters (scale: $20 \mu \mathrm{m}$ ). (D1) Optical projection overview of the posterior tuberculum. (D2) Single plane image. 1 and 2 depicts amplifications of the OXT synapses on TH positive cells in (C) pretectum and (D2) posterior tuberculum. Closed arrowheads point to OXT positive synaptophysin positive synapses that are in direct contact with TH cells. (D3-D4) 6 slice projection (total $6 \mu \mathrm{m}$ thickness) of two different planes (scale: $20 \mu \mathrm{m}$ ). Open arrowheads indicate OXT neuron abutting the TH cells of the posterior Tuberculum. Images taken with Zeiss Ism 810 confocal scanning microscope, scaling $\left(x^{*} y^{*} z\right)=0.156^{*} 0.156^{*} 0.96 \quad(\mu \mathrm{m} / \mathrm{pixel})$, pinhole $=31 \mu \mathrm{m}$, Plan Apochromat 20X/0.8 M27 objective, excitation lasers: green channel (syn-GFP)-488nm; teal (anti-TH) channel-561 nm; magenta (anti-OXT) channel- $640 \mathrm{~nm}$ ). 
Figure 6. Early-life OXT shapes social information processing. (A) Anatomical localization of the two social responsive areas: anterior part of the ventral nucleus of the ventral telencephalon $\left(\mathrm{V} \mathrm{v}_{-} \mathrm{a}, 1\right)$ and anterior part of the parvocellular preoptic nucleus (Ppa, 2). Brain areas identified by DAPI. (B-E) Quantification of the density (cells $/ \mathrm{mm}^{2}$ ) of cells expressing the neuronal marker pS6 in 4-6 days-old MTZ-treated (open squares) or untreated adult fish (full squares), after exposure to either a shoal of conspecifics or an empty tank for $10 \mathrm{~min}$ in the Ppa area (B) and anterior $\mathrm{Vv}$ area (D) and respective representative examples $(C, E)$. Different letters indicate significant statistical differences $(p<0.05)$. $(F)$ Changes in the modular structure of functional connectivity. Modules were obtained by extracting central partition from 400 optimization of Leiden algorithm (Traag et al., 2019) on the treatment correlation matrices. Node color indicates community membership. For visualisation purposes, we only show links with correlation weight $>0.1$. $r$ values indicate the ratio of total edge weight within and between modules. High (low) values of $\boldsymbol{r}$ indicate more (less) segregated modular structure.

Scale: $20 \mu \mathrm{m}$. Data presented as mean \pm SEM. 


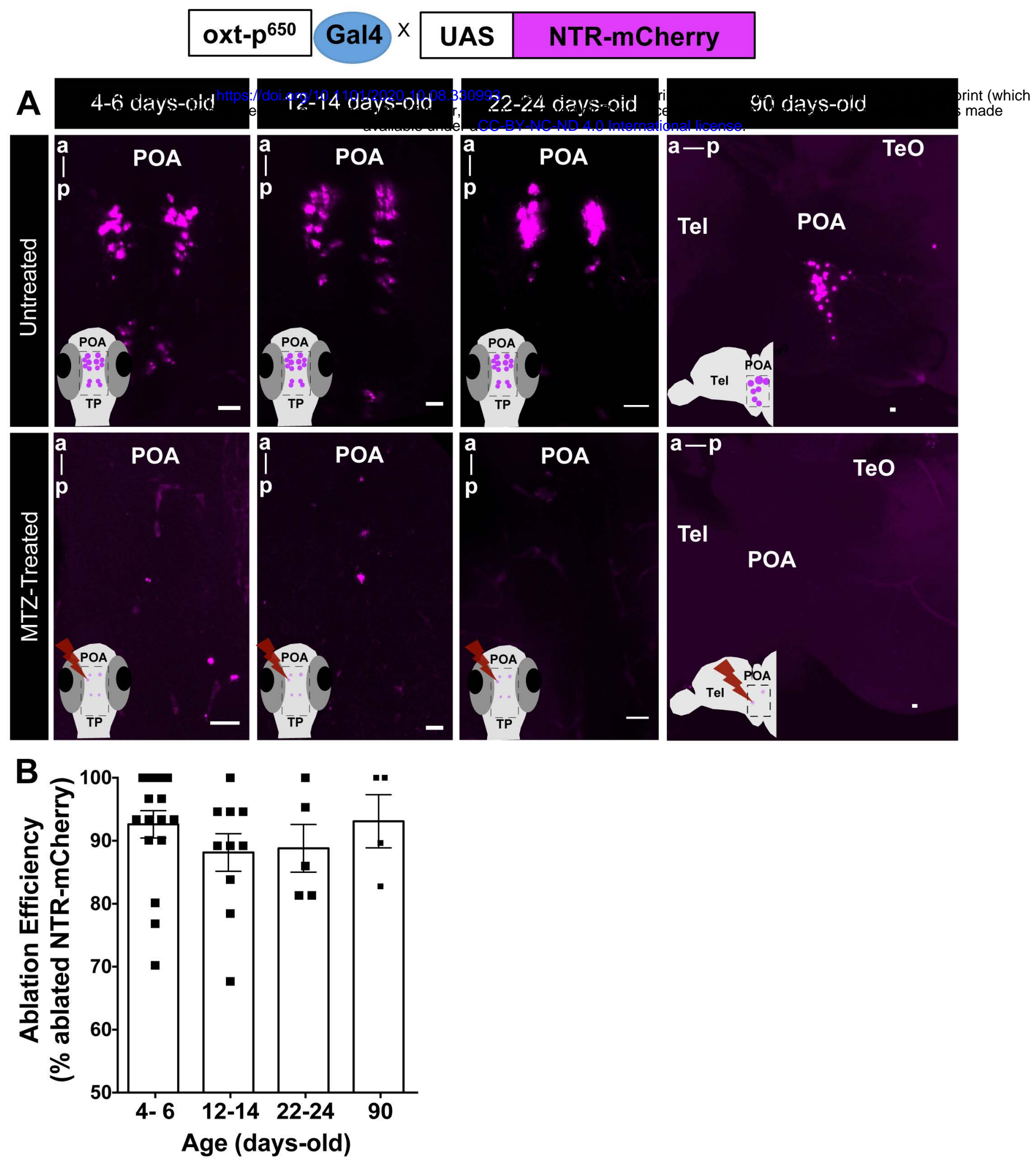


Figure 2-1: Spatio-temporal control of OXT-specific transgene expression.

(A) Representative example of the MTZ-treatment effect on transgene (oxt:GAL4/UASNTR-mCherry) at different treatment time-points. For 4-6, 12-14 and 22-24 days-old: whole mount larvae, maximum intensity confocal z-stack image, dorsal view, anterior to top; for 90 days-old: sagittal brain slice, maximum intensity confocal z-stack image, anterior to left. Scale: $20 \mu \mathrm{m}$. (B) Quantification of the effect of MTZ treatment on transgene expression at all ages tested (4-6 days-old, $n=17 ; 12-14, n=10 ; 22-24$ daysold, $n=5 ; 90$ days-old, $n=4$ ). Ablation efficiency was measured as percentage of ablated cells in MTZ-treated fish over mean number of NTR-mcherry cells in untreated fish. POA, neurosecretory preoptic area; $\mathrm{Tel}$, Telencephalon; $\mathrm{TeO}$, tectum optic. 
A

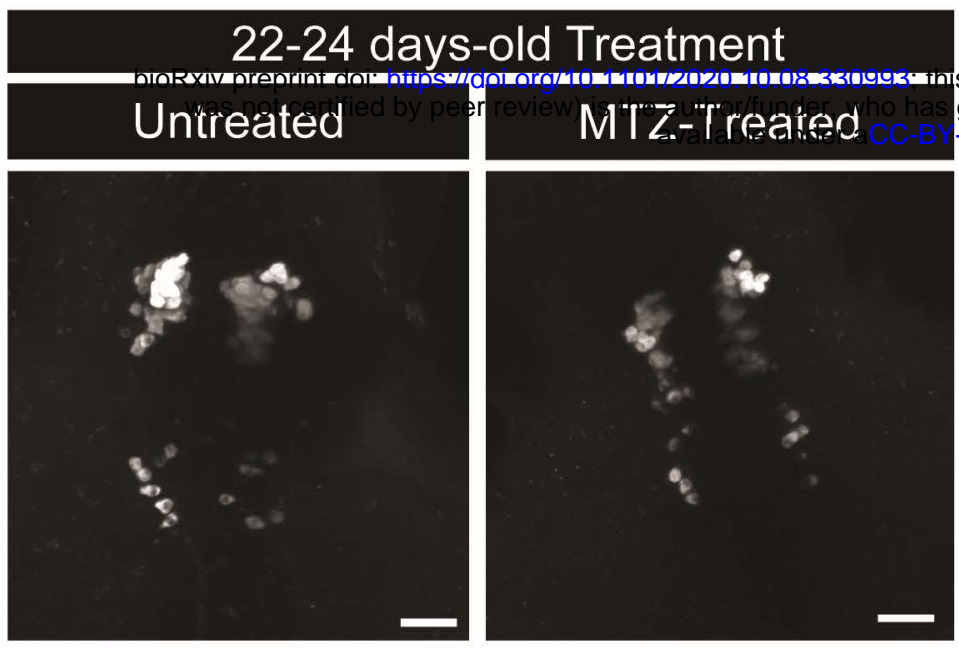

version posted April 29, 2021. The copyright holder for this preprint (which granted bioRxiv a license to display the preprint in perpetuity. It is made -NC-ND 4.0 International license.

B

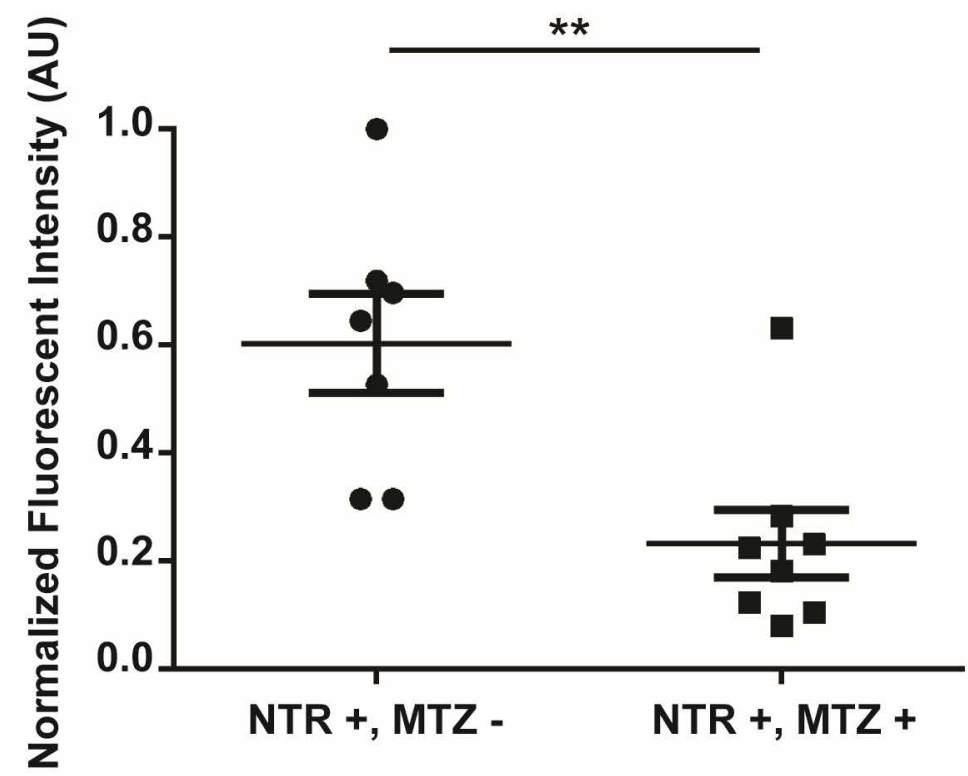


Figure 2-2: Effects of metronidazole (MTZ)- induced ablation on endogenous OXT at 22-24 days-old treatment by in situ hybridization. (A) Representative example of the 22-24 days-old MTZ-treatment effect on endogenous OXT. Whole mount larvae, maximum intensity confocal z-stack image, dorsal view, anterior to top. Scale: $20 \mu \mathrm{m}$. (B) Quantification of normalized fluorescent intensity (AU) in 22-24 days-old untreated (NTR+, MTZ-) or MTZ-treated fish (NTR+, MTZ+). Data presented as mean \pm SEM. ${ }^{* *} \mathrm{p}<0.01$ 


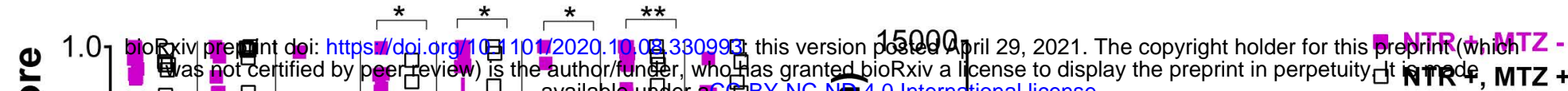
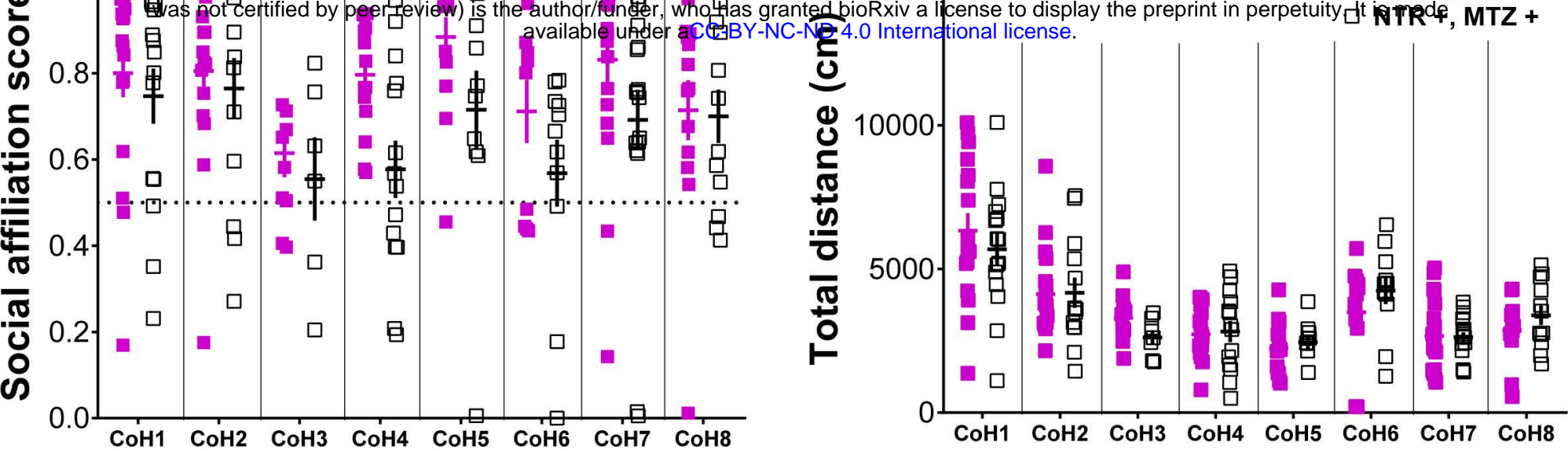

C

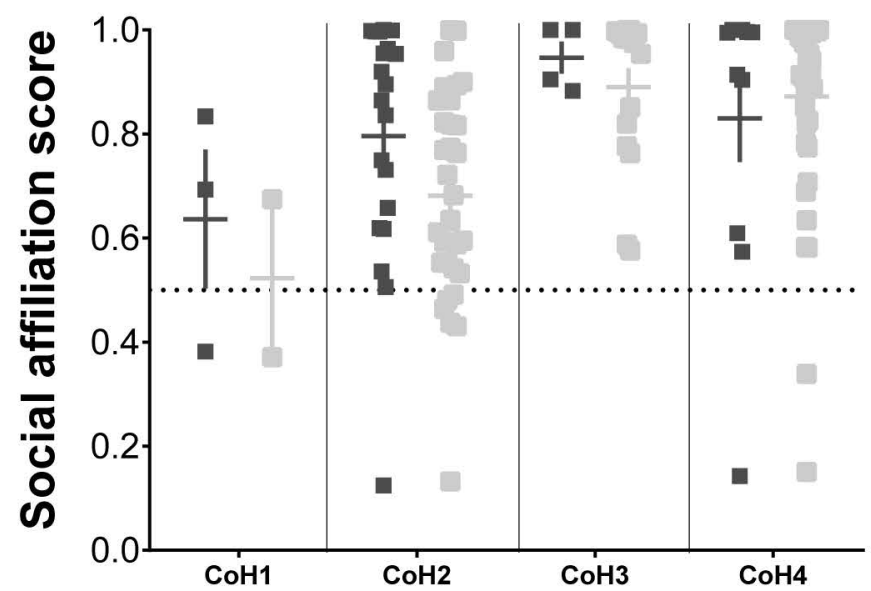

D

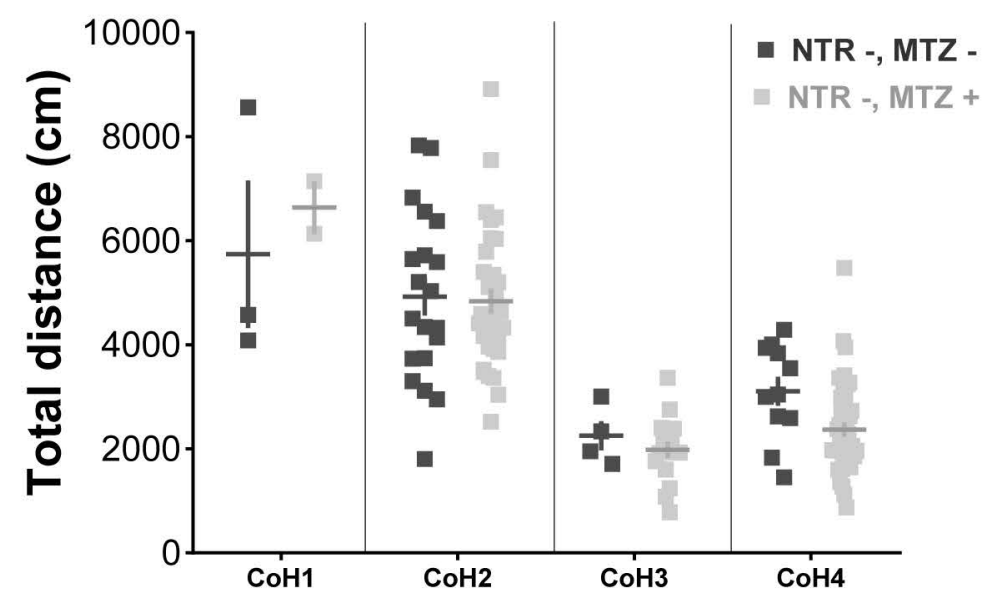


Figure 2-3. Organizational vs activational effects of oxytocin neurons in adult social affiliation. Effect of early (4-6 days-old) MTZ treatment on (A) adult social affiliation and (B) total distance moved of eight independent cohorts of either MTZ treated at 4-6 daysold (NTR+, MTZ+) or untreated control fish (NTR+, MTZ-); (C) adult social affiliation and (D) total distance moved of control cohorts not expressing the transgene, 4-6 days-old MTZ-treated (NTR-, MTZ+) or untreated (NTR-, MTZ-). One-tailed p-values were considered in $(A)$ because of our a priori directionality hypothesis that by ablating OXT neurons, MTZ treatment of fish expressing NTR transgene would decrease social affiliation behavior. Data are presented as mean \pm SEM. Full squares (purple or dark gray): untreated fish (MTZ-); open square or light gray: MTZ-treated fish (MTZ+). 
Figure 2-4. Effects of 4-6 days-old MTZ treatment in adult social affiliation and total distance moved on eight independent experimental cohorts tested

\begin{tabular}{|c|c|c|c|}
\hline \multicolumn{4}{|c|}{ Social affiliation behavior (GLM) } \\
\hline & d.f.1 d.f.2 & F.ratio & p.value \\
\hline Cohort & 7 inf & 2.719 & $0.0081^{* *}$ \\
\hline Treatment & 1 inf & 9.486 & $0.00105^{*}$ \\
\hline Cohort:Treatment & 7 inf & 1.388 & 0.2053 \\
\hline
\end{tabular}

Total Distance moved (LM)

\begin{tabular}{cccccl}
\hline & Df & Sum Sq & Mean Sq & F value & $\operatorname{Pr}(>\mathrm{F})$ \\
\hline Cohort & 7 & 17444 & 2492.03 & 15.2007 & $8.342 \mathrm{e}-16^{* * *}$ \\
\hline Treatment & 1 & 30 & 30.22 & 0.1843 & 0.6681 \\
\hline Cohort: & 7 & 838 & 119.67 & 0.7299 & 0.6468 \\
Treatment & & & & & \\
\hline Residuals & 195 & 31969 & 163.94 & & \\
\hline
\end{tabular}

Summary of results of the GLM (Generalized Linear Model) with beta regression and LM (Linear Model) models to analyse the effects of early (4-6 days-old) MTZ-treatment on adult social affiliation and total distance moved on eight independent experimental cohorts expressing the NTR transgene. A one-tailed $p$-value was considered for the effect of MTZ-treatment on social affiliation behaviour because of our a priori directionality hypothesis (see Methods/Statistic section). 
bioRxiv preprint doi: https://doi.org/10.1101/2020.10.08.330993; this version posted April 29, 2021. The copyright holder for this preprint (which

was not certified by peer review) is the author/funder, who has granted bioRxiv a license to display the preprint in perpetuity. It is made available under aCC-BY-NC-ND 4.0 International license.

Figure 2-4. Effects of 4-6 days-old MTZ treatment in adult social affiliation and total distance moved on eight independent experimental cohorts tested. 
Figure 2-5. Effects of 4-6 days-old MTZ-treatment in adult social affiliation and total distance moved on control fish not expressing the NTR transgene

\begin{tabular}{|c|c|c|c|c|c|}
\hline \multicolumn{6}{|c|}{ Social affiliation behavior (GLM) } \\
\hline & & d.f.1 d.f.2 & \multicolumn{2}{|l|}{2 F.ratio } & p.value \\
\hline Cohort & & 3 inf & 7.249 & & $0.0001^{* * *}$ \\
\hline Treatment & & 1 inf & 1.241 & & 0.2653 \\
\hline Cohort:Trea & nent & 3 inf & 1.271 & & 0.2822 \\
\hline \multicolumn{6}{|c|}{ Total Distance moved (LM) } \\
\hline & $\mathrm{Df}$ & Sum Sq & Mean Sq & F value & $\operatorname{Pr}(>\mathrm{F})$ \\
\hline Cohort & 3 & 15614.2 & 5204.7 & 58.5513 & $<2 e-16^{\star * *}$ \\
\hline Treatment & 1 & 183.3 & 183.3 & 2.0615 & 0.1536 \\
\hline $\begin{array}{l}\text { Cohort: } \\
\text { Treatment }\end{array}$ & 3 & 352.1 & 117.4 & 1.3203 & 0.2710 \\
\hline Residuals & 121 & 10755.9 & 88.9 & & \\
\hline
\end{tabular}

Summary of results of the GLM with beta regression and LM models to analyse the effects of early (4-6 days-old) MTZ-treatment on adult social affiliation and total distance moved on control fish not expressing the NTR transgene. 
bioRxiv preprint doi: https://doi.org/10.1101/2020.10.08.330993; this version posted April 29, 2021. The copyright holder for this preprint (which

was not certified by peer review) is the author/funder, who has granted bioRxiv a license to display the preprint in perpetuity. It is made available under aCC-BY-NC-ND 4.0 International license.

Figure 2-5. Effects of 4-6 days-old MTZ-treatment in adult social affiliation and total distance moved on control fish not expressing the NTR transgene. 


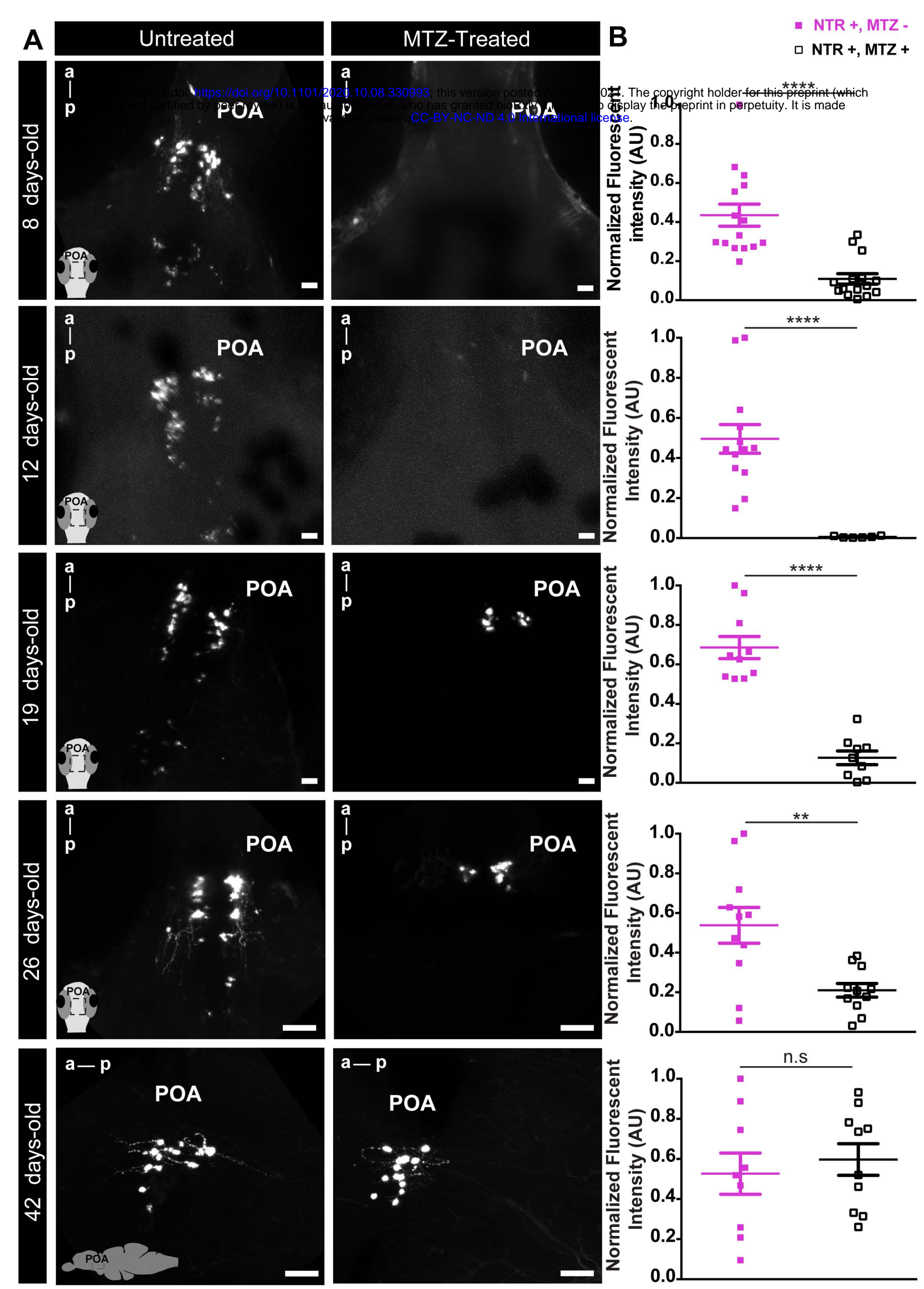


Figure 2-6. Time-course OXT:NTR-mCherry cell recovery after 4-6 days-old MTZtreatment. 4-6 days-old metronidazole (MTZ)- treated vs untreated larvae were left to grow and sampled at different time-points throughout development: 8, 12, 19, 26, and 42 days-old. A) Representative whole mount larvae, maximum intensity confocal z-stack image, dorsal view, anterior to top (8, 12, 19 and 26 days-old) and sagittal brain slice, confocal z-stack image, anterior to left (42 days-old). B) Quantification of the Normalized fluorescent intensity (AU) in untreated (full squares) versus MTZ-treated fish (open squares) at the different time points sampled.

Scale: $20 \mu \mathrm{m}$ (8-12-19-days-old) and $50 \mu \mathrm{m}$ (26 and 42 days-old). Data are presented as mean \pm SEM. Full squares: untreated fish (NTR+,MTZ-); open square: MTZ-treated fish $(\mathrm{NTR}+, \mathrm{MTZ}+) .{ }^{* *} p<0.01,{ }^{* * * *} p<0.0001$ 

a - p s pho preprint in perpetuity. It is made
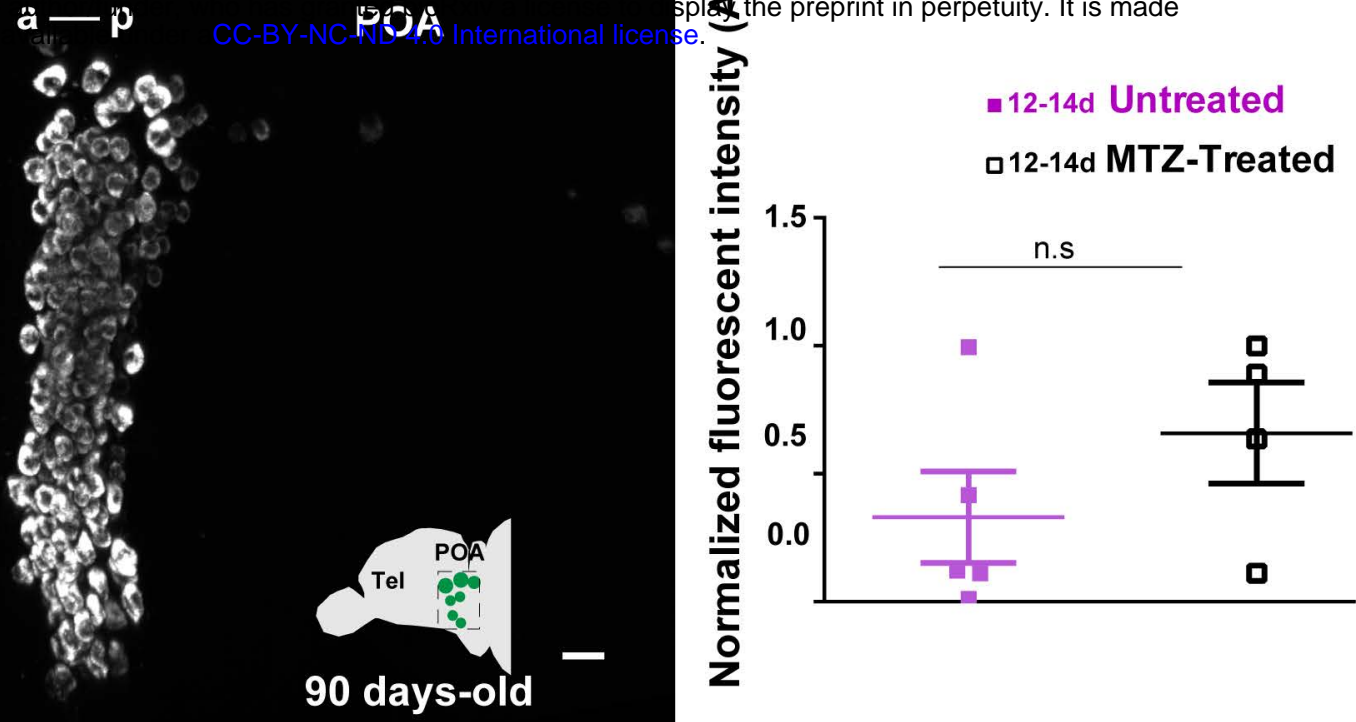

든 
Figure 2-7. Endogenous OXT mRNA recovery in adulthood after 12-14 days-old MTZ-treatment. (A) Representative example of the endogenous OXT mRNA in untreated adult fish. (B) Representative example of the endogenous OXT mRNA in adult fish treated with MTZ at 12-14 days-old. Images in $(A, B)$ are maximum intensity confocal z-stacks, sagittal slices, anterior to left. Scale: $20 \mu \mathrm{m}$. (C) Quantification of normalized fluorescent intensity (AU) in untreated (full squares) and 12-14 days-old MTZ-treated adult fish (empty squares). Data presented as mean \pm SEM. Full squares: untreated fish (NTR+,MTZ-); open square: MTZ-treated fish (NTR+,MTZ+). 


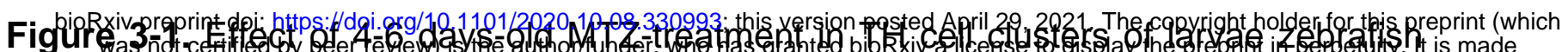

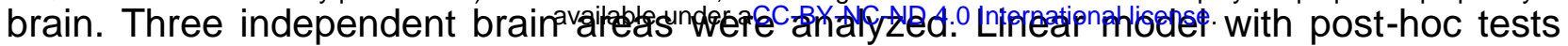
comparing the treatment (early MTZ-treated vs. untreated) were performed.

\begin{tabular}{lcccccc}
\hline \multicolumn{1}{c}{ BA } & Treat1 & Treat2 & estim & SE & z.ratio & p.value \\
\hline Subpallium & Untreated & MTZ-Treated & -2.889 & 2.622 & -1.102 & 0.278 \\
\hline PrT & Untreated & MTZ-Treated & -8.278 & 2.994 & -2.764 & $0.009^{* *}$ \\
\hline TP Large cells & Untreated & MTZ-Treated & -1.722 & 0.566 & -3.042 & $0.0045^{* *}$ \\
\hline
\end{tabular}

BA, Brain area; Treat, Treatment; estim, estimate; PrT, Pretectum; TP, Posterior Tuberculum. 
bioRxiv preprint doi: https://doi.org/10.1101/2020.10.08.330993; this version posted April 29, 2021. The copyright holder for this preprint (which

was not certified by peer review) is the author/funder, who has granted bioRxiv a license to display the preprint in perpetuity. It is made available under aCC-BY-NC-ND 4.0 International license.

Figure 3-1. Effect of 4-6 days-old MTZ-treatment in TH cell clusters of larvae zebrafish brain. Three independent brain areas were analyzed. Linear model with posthoc tests comparing the treatment (early MTZ-treated vs. untreated) were performed. 


\section{A}

B

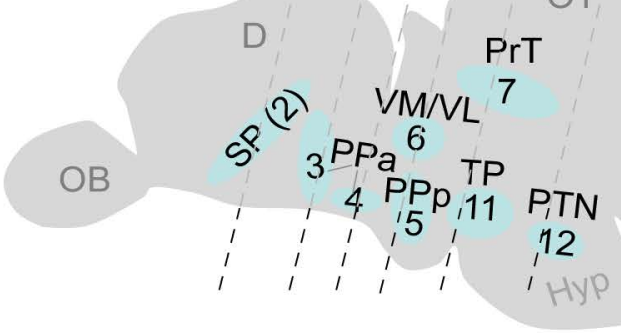

\section{anti-TH DAPI}
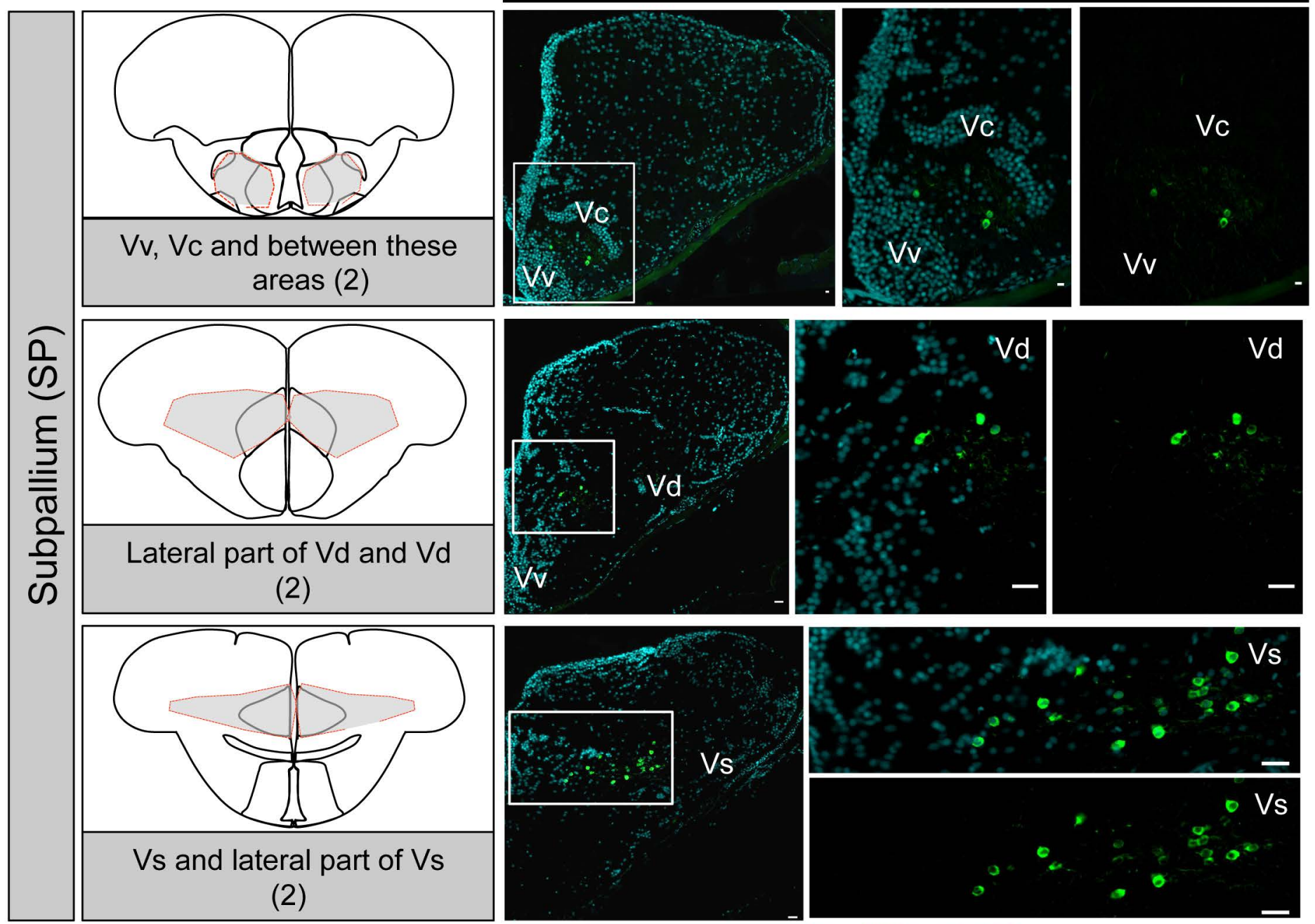

Vd
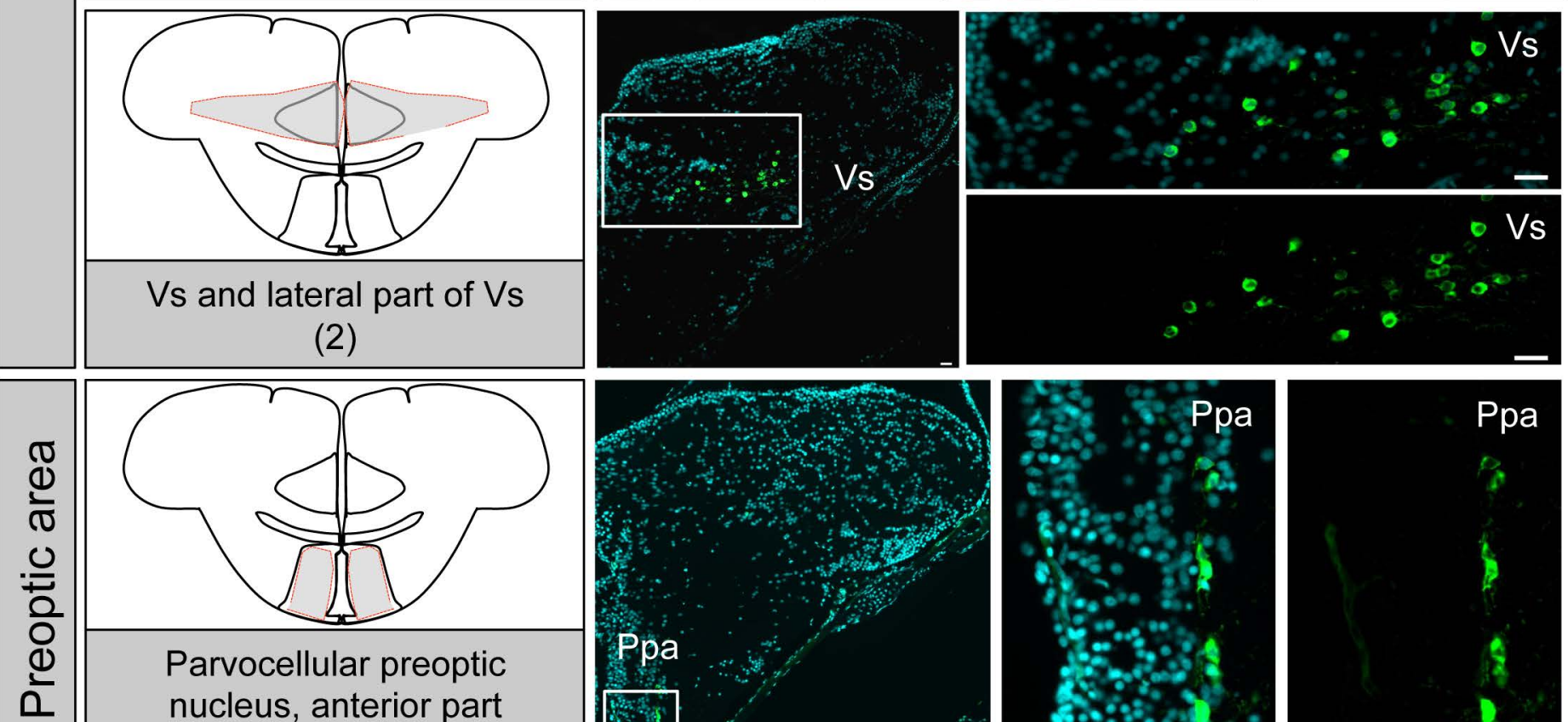

Parvocellular preoptic nucleus, anterior part (Ppa 3)
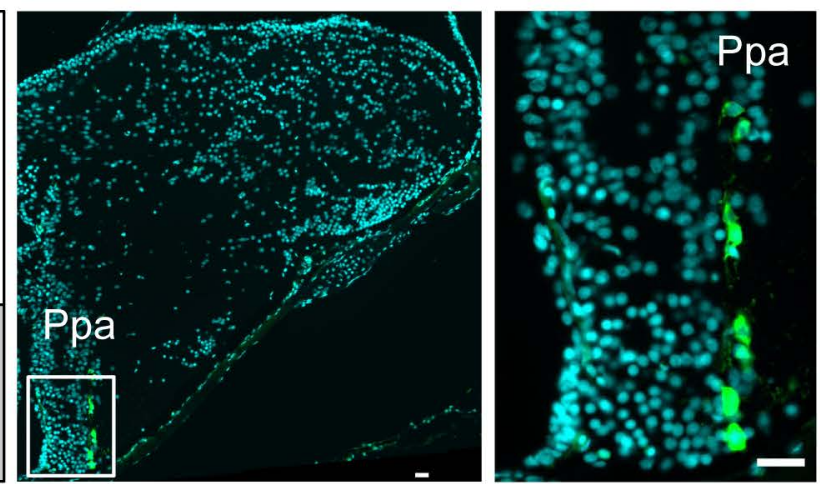

Ppa

3

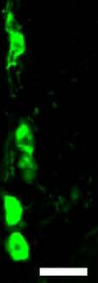


anti-TH DAPI
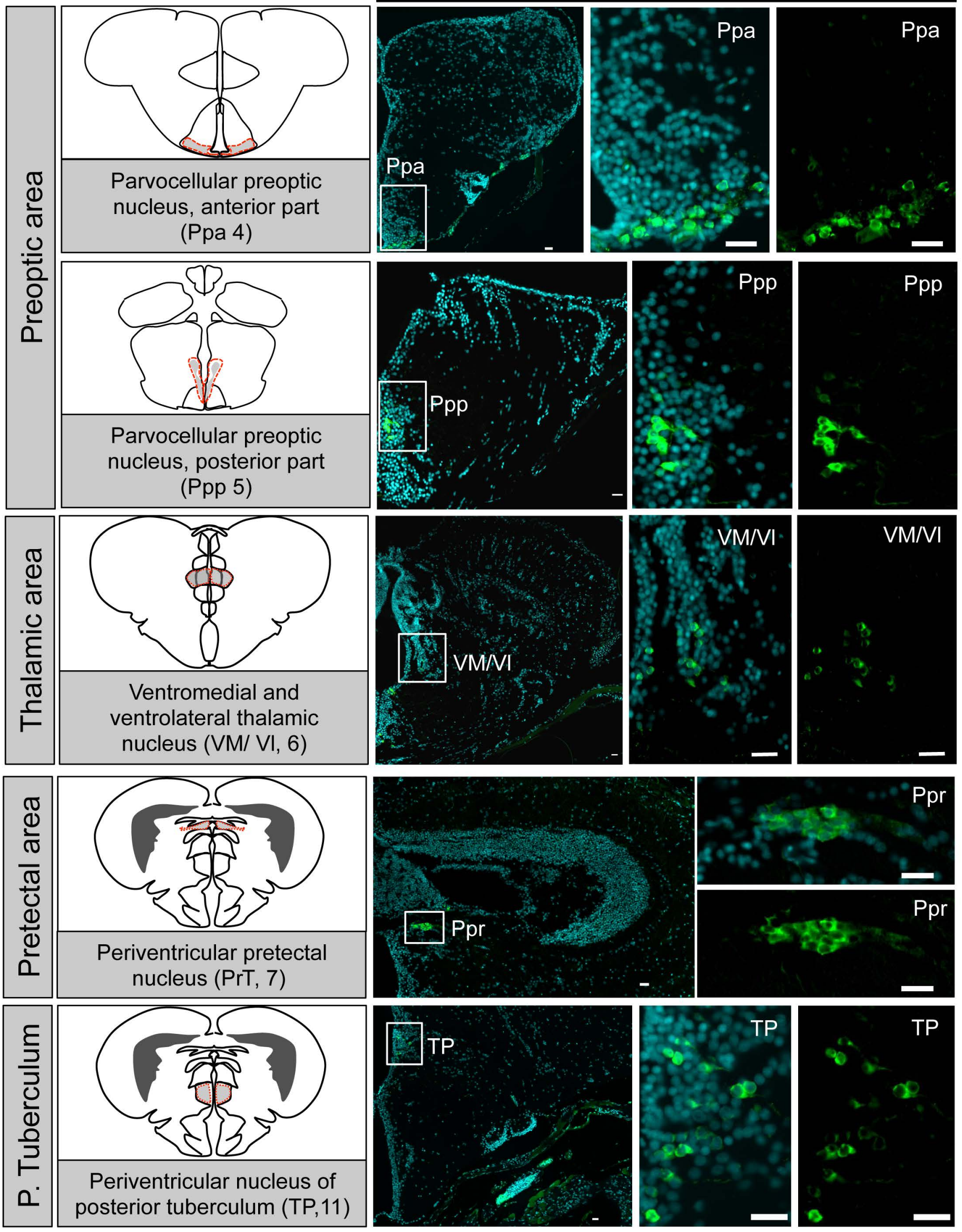


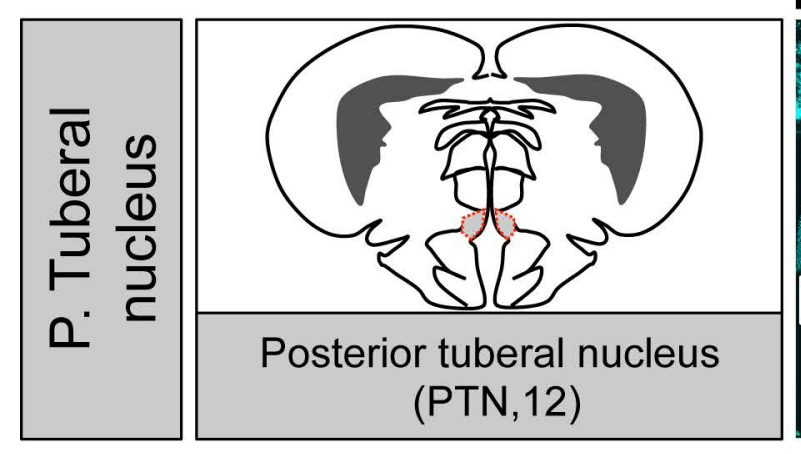

anti-TH DAPI

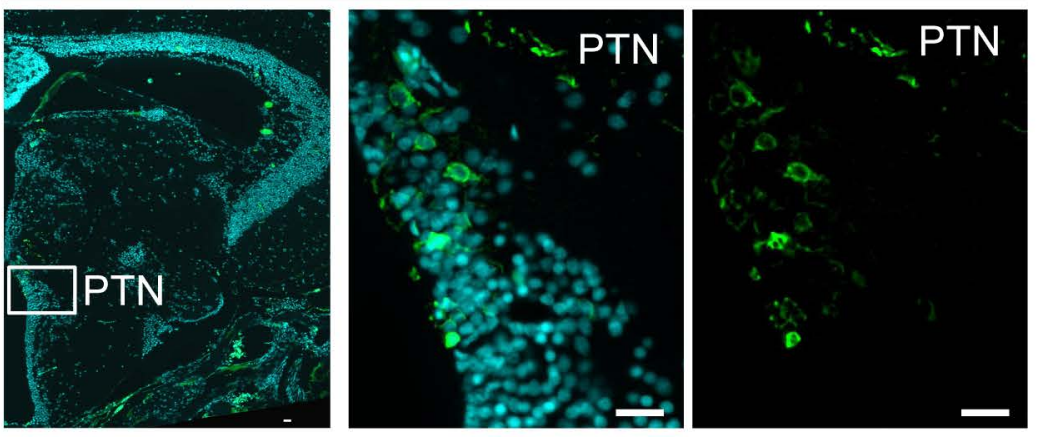


Figure 4-1 - Early life OXT ablation affects the dopaminergic system. (A) Schematic representation of an adult zebrafish sagittal view representing the coronal sections of all dopaminergic areas sampled in the adult brain (dopaminergic cluster nomenclature according to(Panula et al., 2010)). (B) Schematic representation of zebrafish brain coronal sections highlighting the different dopaminergic clusters (adapted from (Wullimann et al., 1996)) and representative example showing anatomical localization of the dopaminergic groups in an untreated adult zebrafish. Landmarks of the areas identified by DAPI (cyan) and dopaminergic groups by TH (Tyrosine Hydroxylase) immunostaining (green). Scale bars are $20 \mu \mathrm{m}$. 


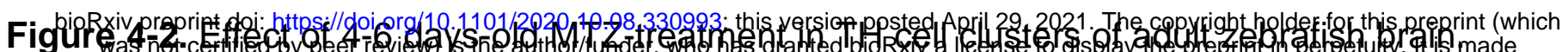

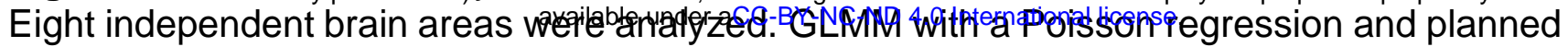
comparisons (early MTZ-Treated vs. Untreated) was performed. p-values from planned comparisons were corrected with false discovery rate (FDR) adjustment method.

\begin{tabular}{lccrrrrc}
\hline \multicolumn{1}{c}{ BA } & Treat1 & Treat2 & estim & SE & z.ratio & $\begin{array}{c}\text { p.value. } \\
\text { indep. } \\
\text { GLMM }\end{array}$ & $\begin{array}{c}\text { p.value. } \\
\text { indep. GLMM. } \\
\text { fdr correction }\end{array}$ \\
\hline Subpallium (G2) & Untreated & MTZ-Treated & -0.031 & 0.0562 & -0.552 & 0.5813 & 0.6643 \\
\hline $\mathrm{PPa}(\mathrm{G} 3)$ & Untreated & MTZ-Treated & 0.185 & 0.175 & 1.058 & 0.2901 & 0.3868 \\
\hline $\mathrm{PPa}(\mathrm{G} 4)$ & Untreated & MTZ-Treated & 0.0611 & 0.0533 & 1.147 & 0.2513 & 0.3868 \\
\hline $\mathrm{PPp}(\mathrm{G} 5)$ & Untreated & MTZ-Treated & 0.12 & 0.0747 & 1.603 & 0.1090 & 0.218 \\
\hline $\mathrm{Vm}+\mathrm{VI}(\mathrm{G} 6)$ & Untreated & MTZ-Treated & 0.0143 & 0.0755 & 0.189 & 0.8502 & 0.8502 \\
\hline $\mathrm{PrT}(\mathrm{G} 7)$ & Untreated & MTZ-Treated & 0.201 & 0.0598 & 3.364 & 0.0008 & $0.0048^{* *}$ \\
\hline $\mathrm{TP}$ (G11) & Untreated & MTZ-Treated & 0.188 & 0.0724 & 2.597 & 0.0094 & $0.0251^{*}$ \\
\hline $\mathrm{PTN}(\mathrm{G} 12)$ & Untreated & MTZ-Treated & 0.19 & 0.0586 & 3.239 & 0.0012 & $0.0048^{* *}$ \\
\hline
\end{tabular}

$B A$, Brain area; Treat, Treatment; estim, estimate; GLMM, Generalized Linear Mixed Models; $F D R$, false discovery rate, $P P a$, anterior part of the parvocellular preoptic nucleus; $P P p$, posterior part of the Parvocellular preoptic nucleus; $V m$, ventromedial thalamic nucleus; $V I$, ventrolateral thalamic nucleus; PrT, Pretectum nucleus; TP, Posterior tuberculum nucleus; PTN, Posterior Tuberal nucleus. 
Figure 4-2. Effect of 4-6 days-old MTZ-treatment in TH cell clusters of adult zebrafish brain. Eight independent brain areas were analyzed. GLMM with a Poisson regression and planned comparisons (early MTZ-Treated vs. Untreated) was performed. $p$-values from planned comparisons were corrected with false discovery rate (FDR) adjustment method. 


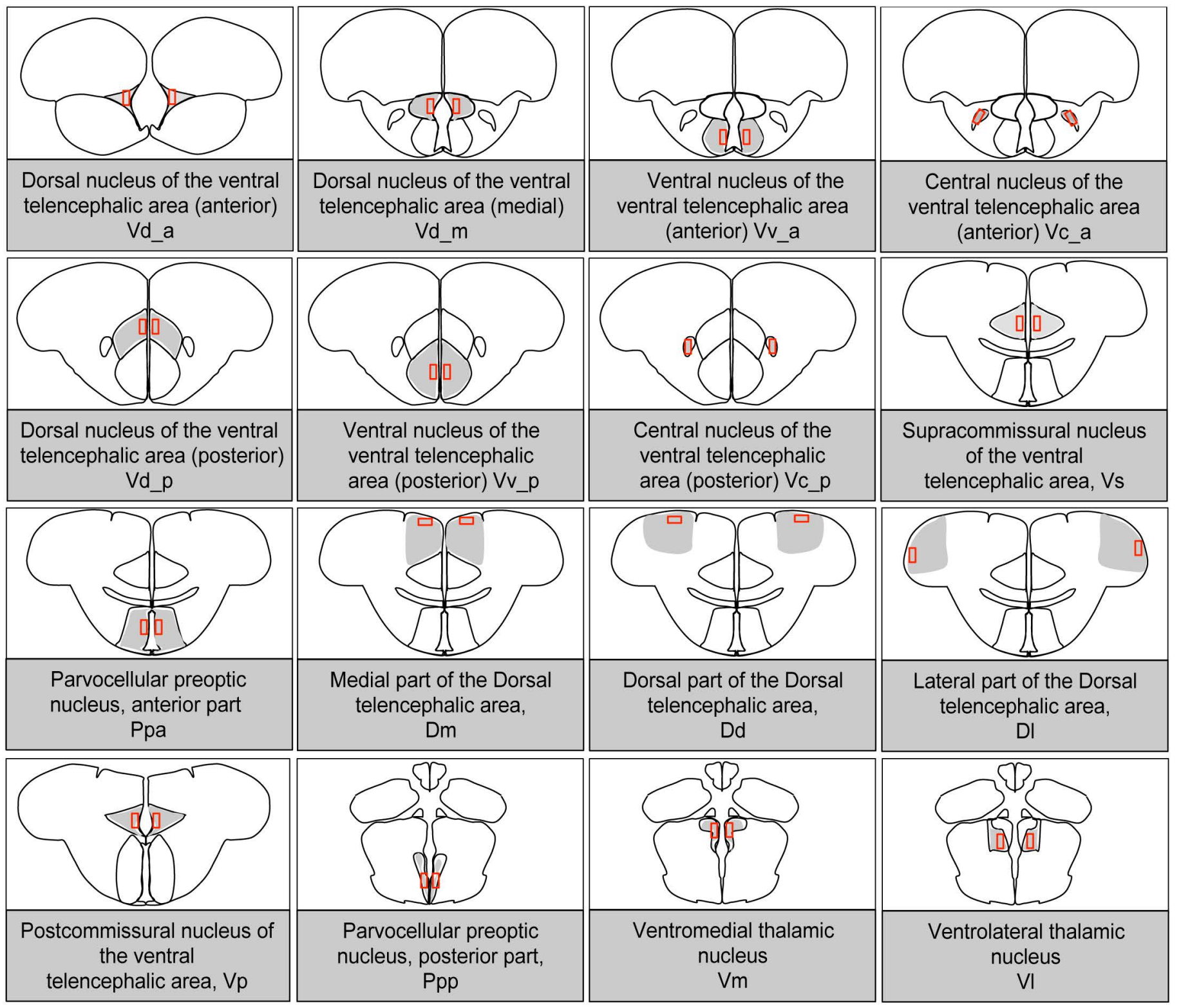


Figure 6-1. Schematic representation of brain areas that were analyzed for neuronal activation in response to social stimulus. pS6 immunostaining served as a readout of neuronal activation following MTZ treatment at 4-6 days-old or untreated adult fish to either a shoal of conspecifics or an empty tank. Included brain areas are part of the social decision-making network, subdivided into more anterior or posterior parts. Schematics are adapted from zebrafish atlas (Wullimann et al., 1996). 


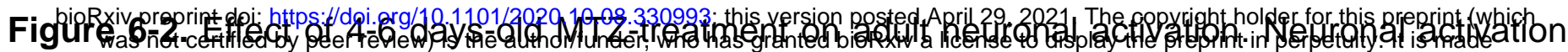

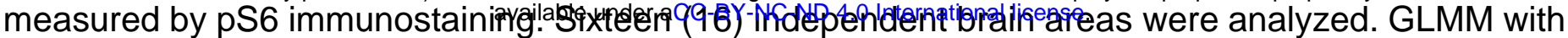
a Poisson regression and planned comparisons were performed. $p$-values of the planned comparisons were corrected with false discovery rate (FDR) adjustment method (significant values in red).

\begin{tabular}{|c|c|c|c|c|c|c|c|c|c|c|}
\hline BA1 & Stim1 & Treat1 & BA2 & Stim2 & Treat2 & estim & SE & z.ratio & $\begin{array}{l}\text { p.value. } \\
\text { indep. } \\
\text { GLMM }\end{array}$ & $\begin{array}{l}\text { p.value. } \\
\text { indep. GLMM. } \\
\text { fdr correction }\end{array}$ \\
\hline $\mathrm{Dd}$ & EMPTY & CTR & Dd & SHOAL & CTR & -0.2076 & 0.125 & -1.655 & 0.0978 & 0.4084 \\
\hline $\mathrm{Dd}$ & EMPTY & ABLAT & Dd & EMPTY & CTR & -0.2057 & 0.147 & -1.399 & 0.1618 & 0.523687 \\
\hline $\mathrm{Dd}$ & SHOAL & ABLAT & $\mathrm{Dd}$ & SHOAL & CTR & -0.0078 & 0.118 & -0.066 & 0.9477 & 0.9683302 \\
\hline $\mathrm{Dd}$ & EMPTY & ABLAT & $\mathrm{Dd}$ & SHOAL & ABLAT & -0.0096 & 0.138 & -0.069 & 0.9448 & 0.9683302 \\
\hline $\mathrm{DL}$ & EMPTY & CTR & $\mathrm{DL}$ & SHOAL & CTR & -0.096 & 0.0948 & -1.013 & 0.311 & 0.7052 \\
\hline $\mathrm{DL}$ & EMPTY & ABLAT & $\mathrm{DL}$ & EMPTY & CTR & -0.1449 & 0.1067 & -1.359 & 0.1743 & 0.523687 \\
\hline $\mathrm{DL}$ & SHOAL & ABLAT & $\mathrm{DL}$ & SHOAL & CTR & -0.1496 & 0.0831 & -1.8 & 0.0718 & 0.4084 \\
\hline $\mathrm{DL}$ & EMPTY & ABLAT & $\mathrm{DL}$ & SHOAL & ABLAT & -0.1007 & 0.0964 & -1.044 & 0.2964 & 0.7025778 \\
\hline DM & EMPTY & CTR & DM & SHOAL & CTR & -0.0206 & 0.118 & -0.174 & 0.8617 & 0.9347254 \\
\hline DM & EMPTY & ABLAT & DM & EMPTY & CTR & -0.0238 & 0.135 & -0.177 & 0.8598 & 0.9347254 \\
\hline DM & SHOAL & ABLAT & DM & SHOAL & CTR & -0.1401 & 0.105 & -1.332 & 0.1827 & 0.523687 \\
\hline DM & EMPTY & ABLAT & DM & SHOAL & ABLAT & -0.1369 & 0.124 & -1.107 & 0.2683 & 0.6604308 \\
\hline PPA & EMPTY & CTR & PPA & SHOAL & CTR & -0.2858 & 0.0899 & -3.18 & 0.0015 & 0.024 \\
\hline PPA & EMPTY & ABLAT & PPA & EMPTY & CTR & 0.3415 & 0.1018 & 3.356 & 0.0008 & 0.024 \\
\hline PPA & SHOAL & ABLAT & PPA & SHOAL & CTR & 0.017 & 0.0793 & 0.215 & 0.83 & 0.9347254 \\
\hline PPA & EMPTY & ABLAT & PPA & SHOAL & ABLAT & 0.0387 & 0.0926 & 0.418 & 0.6761 & 0.8830694 \\
\hline PPp & EMPTY & CTR & PPp & SHOAL & CTR & -0.2579 & 0.116 & -2.229 & 0.0258 & 0.2638222 \\
\hline PPp & EMPTY & ABLAT & PPp & EMPTY & CTR & -0.1959 & 0.129 & -1.521 & 0.1284 & 0.4833882 \\
\hline PPp & SHOAL & ABLAT & PPp & SHOAL & CTR & -0.0306 & 0.103 & -0.297 & 0.7663 & 0.9082074 \\
\hline PPp & EMPTY & ABLAT & PPp & SHOAL & ABLAT & -0.0926 & 0.117 & -0.789 & 0.4302 & 0.795241 \\
\hline VC_a & EMPTY & CTR & VC_a & SHOAL & CTR & 0.0668 & 0.141 & 0.474 & 0.6358 & 0.8830694 \\
\hline VC_a & EMPTY & ABLAT & VC_a & EMPTY & CTR & -0.3189 & 0.153 & -2.085 & 0.0371 & 0.2638222 \\
\hline VC_a & SHOAL & ABLAT & VC_a & SHOAL & CTR & -0.2749 & 0.132 & -2.085 & 0.0371 & 0.2638222 \\
\hline VC_a & EMPTY & ABLAT & VC_a & SHOAL & ABLAT & 0.1107 & 0.144 & 0.767 & 0.4433 & 0.795241 \\
\hline VC_p & EMPTY & CTR & VC_p & SHOAL & CTR & -0.0203 & 0.133 & -0.153 & 0.8786 & 0.9371733 \\
\hline VC_p & EMPTY & ABLAT & VC_p & EMPTY & CTR & -0.1035 & 0.146 & -0.708 & 0.4789 & 0.795241 \\
\hline VC_p & SHOAL & ABLAT & VC_p & SHOAL & CTR & -0.0401 & 0.116 & -0.347 & 0.7285 & 0.9082074 \\
\hline VC_p & EMPTY & ABLAT & VC_p & SHOAL & ABLAT & 0.0431 & 0.131 & 0.33 & 0.7417 & 0.9082074 \\
\hline VD_a & EMPTY & CTR & VD_a & SHOAL & CTR & -0.2073 & 0.161 & -1.287 & 0.1981 & 0.5282667 \\
\hline VD_a & EMPTY & ABLAT & VD_a & EMPTY & CTR & -0.1121 & 0.17 & -0.659 & 0.5102 & 0.81632 \\
\hline VD_a & SHOAL & ABLAT & VD_a & SHOAL & CTR & 0.0089 & 0.152 & 0.059 & 0.9532 & 0.9683302 \\
\hline VD_a & EMPTY & ABLAT & VD_a & SHOAL & ABLAT & -0.0862 & 0.162 & -0.533 & 0.5942 & 0.8830694 \\
\hline VD_m & EMPTY & CTR & VD_m & SHOAL & CTR & 0.0482 & 0.115 & 0.419 & 0.6749 & 0.8830694 \\
\hline VD_m & EMPTY & ABLAT & VD_m & EMPTY & CTR & -0.2684 & 0.125 & -2.147 & 0.0318 & 0.2638222 \\
\hline VD_m & SHOAL & ABLAT & VD_m & SHOAL & CTR & -0.0759 & 0.109 & -0.699 & 0.4846 & 0.795241 \\
\hline VD_m & EMPTY & ABLAT & VD_m & SHOAL & ABLAT & 0.2407 & 0.119 & 2.016 & 0.0438 & 0.28032 \\
\hline VD_p & EMPTY & CTR & VD_p & SHOAL & CTR & 0.112 & 0.114 & 0.985 & 0.3247 & 0.7052 \\
\hline VD_p & EMPTY & ABLAT & VD_p & EMPTY & CTR & -0.169 & 0.125 & -1.355 & 0.1754 & 0.523687 \\
\hline VD_p & SHOAL & ABLAT & VD_p & SHOAL & CTR & -0.173 & 0.104 & -1.661 & 0.0968 & 0.4084 \\
\hline VD_p & EMPTY & ABLAT & VD_p & SHOAL & ABLAT & 0.108 & 0.116 & 0.932 & 0.3513 & 0.7052 \\
\hline $\mathrm{VL}$ & EMPTY & CTR & $\mathrm{VL}$ & SHOAL & CTR & 0.00959 & 0.328 & 0.029 & 0.9767 & 0.9767 \\
\hline VL & EMPTY & ABLAT & $\mathrm{VL}$ & EMPTY & CTR & 0.06954 & 0.365 & 0.19 & 0.8491 & 0.9347254 \\
\hline
\end{tabular}




\begin{tabular}{|c|c|c|c|c|c|c|c|c|c|c|}
\hline $\mathrm{VL}$ & EMPTY & ABLAT & $\mathrm{VL}$ & bdghOAder & AB'YAT & $40.0809 y^{2}$ & 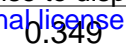 & 0.232 & 0.8165 & 0.9347254 \\
\hline VM & EMPTY & CTR & VM & SHOAL & CTR & -0.3257 & 0.265 & -1.231 & 0.2184 & 0.559104 \\
\hline VM & EMPTY & ABLAT & VM & EMPTY & CTR & 0.1426 & 0.299 & 0.476 & 0.6339 & 0.8830694 \\
\hline VM & SHOAL & ABLAT & VM & SHOAL & CTR & 0.3621 & 0.248 & 1.461 & 0.144 & 0.512 \\
\hline VM & EMPTY & ABLAT & VM & SHOAL & ABLAT & -0.1062 & 0.285 & -0.373 & 0.7089 & 0.907392 \\
\hline VP & EMPTY & CTR & VP & SHOAL & CTR & -0.2041 & 0.123 & -1.663 & 0.0963 & 0.4084 \\
\hline VP & EMPTY & ABLAT & VP & EMPTY & CTR & -0.1158 & 0.145 & -0.8 & 0.4234 & 0.795241 \\
\hline VP & SHOAL & ABLAT & VP & SHOAL & CTR & 0.1484 & 0.113 & 1.316 & 0.1882 & 0.523687 \\
\hline VP & EMPTY & ABLAT & VP & SHOAL & ABLAT & 0.06 & 0.135 & 0.446 & 0.6559 & 0.8830694 \\
\hline VS & EMPTY & CTR & VS & SHOAL & CTR & -0.3116 & 0.117 & -2.664 & 0.0077 & 0.09856 \\
\hline VS & EMPTY & ABLAT & VS & EMPTY & CTR & -0.1251 & 0.133 & -0.938 & 0.3483 & 0.7052 \\
\hline VS & SHOAL & ABLAT & VS & SHOAL & CTR & 0.0968 & 0.104 & 0.93 & 0.3526 & 0.7052 \\
\hline VS & EMPTY & ABLAT & VS & SHOAL & ABLAT & -0.0897 & 0.122 & -0.734 & 0.4628 & 0.795241 \\
\hline VV_a & EMPTY & CTR & $V V_{-} a$ & SHOAL & CTR & 0.6076 & 0.19 & 3.193 & 0.0014 & 0.024 \\
\hline$V V_{-} a$ & EMPTY & ABLAT & VV_a & EMPTY & CTR & 0.0626 & 0.205 & 0.305 & 0.7606 & 0.9082074 \\
\hline$V V_{-} a$ & SHOAL & ABLAT & $V V_{-} a$ & SHOAL & CTR & -0.6333 & 0.181 & -3.504 & 0.0005 & 0.024 \\
\hline VV_a & EMPTY & ABLAT & VV_a & SHOAL & ABLAT & -0.0883 & 0.197 & -0.449 & 0.6537 & 0.8830694 \\
\hline VV_p & EMPTY & CTR & $V V \_p$ & SHOAL & CTR & 0.0579 & 0.114 & 0.509 & 0.611 & 0.8830694 \\
\hline$V V \_p$ & EMPTY & ABLAT & $V V \_p$ & EMPTY & CTR & -0.2034 & 0.124 & -1.635 & 0.1021 & 0.4084 \\
\hline VV_p & SHOAL & ABLAT & VV_p & SHOAL & CTR & -0.1748 & 0.104 & -1.687 & 0.0915 & 0.4084 \\
\hline$V V \_p$ & EMPTY & ABLAT & $V V \_p$ & SHOAL & ABLAT & 0.0865 & 0.115 & 0.751 & 0.4529 & 0.795241 \\
\hline
\end{tabular}

BA1, Brain area 1; BA2, Brain area 2; Stim, stimulus; Treat, Treatment; estim, estimate; indep., independent, GLMM, Generalized Linear Mixed Models; FDR, false discovery rate, CTR, untreated fish; $A B L T$, early MTZ-treated fish; $V v_{-} a$, anterior part of the ventral nucleus of the ventral telencephalic area $(\mathrm{V}) ; V v \_p$, posterior part of the ventral nucleus of the ventral telencephalic area $(\mathrm{V})$; $V d \_a$, anterior part of the dorsal nucleus of $\mathrm{V}$; $V d \_m$, medial part of the dorsal nucleus of $\mathrm{V} ; V d \_p$, posterior part of the dorsal nucleus of $\mathrm{V} ; \mathrm{V} c \_a$, anterior part of the central nucleus of $\mathrm{V} ; \mathrm{V} \_\_$, posterior part of the central nucleus of $\mathrm{V}$; $D m$, medial zone of the dorsal telencephalic area (D); $D$, lateral zone of the dorsal telencephalic area (D); $D d$, dorsal zone of the dorsal telencephalic area (D); Vs, supracommissural nucleus of $\mathrm{V} ; V p$, postcommissural nucleus of $\mathrm{V}$; Ppa, anterior part of the parvocellular preoptic nucleus; Ppp, posterior part of the parvocellular preoptic nucleus; VM, ventromedial thalamic nucleus; $V L$, ventrolateral thalamic nucleus. 
Figure 6-2. Effect of 4-6 days-old MTZ-treatment on adult neuronal activation. Neuronal activation measured by pS6 immunostaining. Sixteen (16) independent brain areas were analyzed. GLMM with a Poisson regression and planned comparisons were performed. p-values of the planned comparisons were corrected with false discovery rate (FDR) adjustment method (significant values in red). 
A

B

VD_a -

VD_mvo_a -

W__a - bioRxiv preprint doi: https://doi.org/10.1101/2020.10.08.33 ${ }^{0.7}$

VD $m$

, this version postadaApril 29, 2021. The copyright holder for this preprint (which

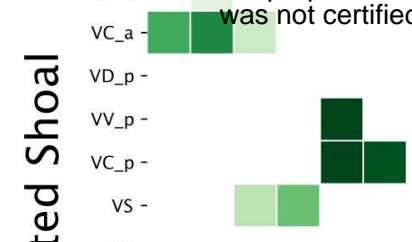

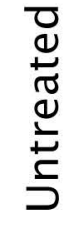

VP -

PPA -

DM -

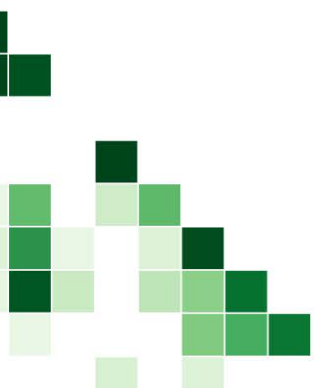

has granted bioRxiv a license to display the preprint in perpetuity. It is made

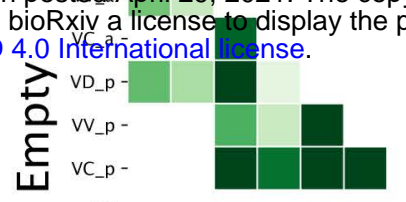

$-0.5$

DL -

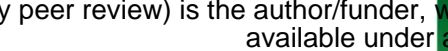

Ce-BY-NC-ND 4.0 international license.

VM -

$\mathrm{VL}$ -

PPp -

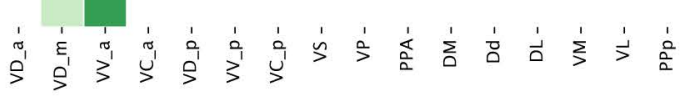

C

$$
\text { E }
$$

vDa-

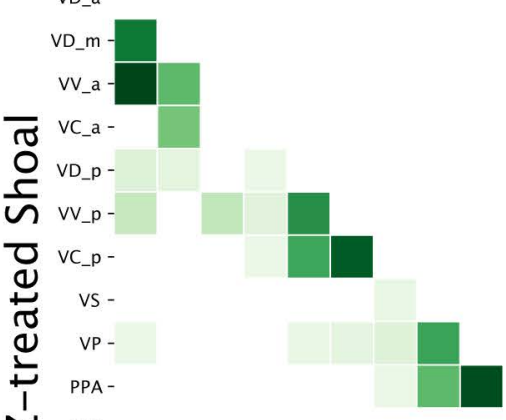

D

VD_a -

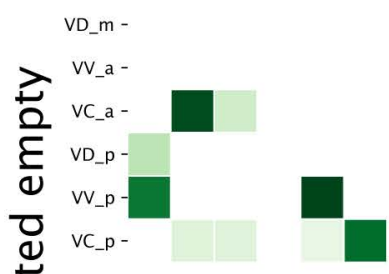

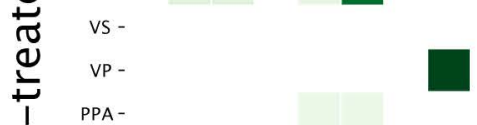

$\mathrm{N}$ DM-

$\stackrel{N}{\Sigma}$

DM

Dd -

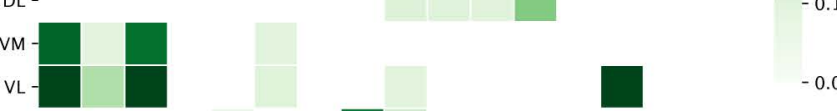

PPp -

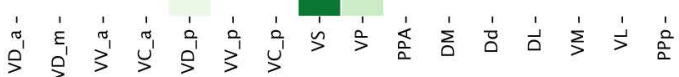

$-0$.

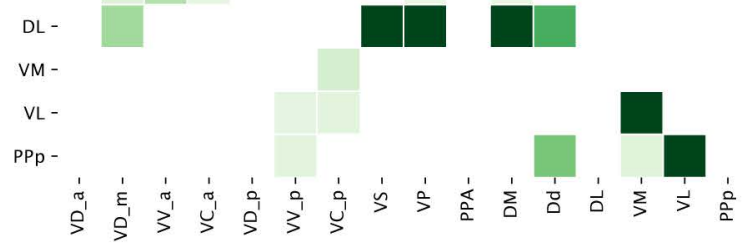

$-0.1$ 
Figure 6-3. Correlation matrices of functional connectivity for the four treatments reveal early-life OXT shapes social information processing. Regional correlation values are computed joining data from both hemispheres. Resampled correlations were kept if significant $(p<0.05)$. For visualisation purposes, we show only entries of significant correlations higher than 0.05 . 


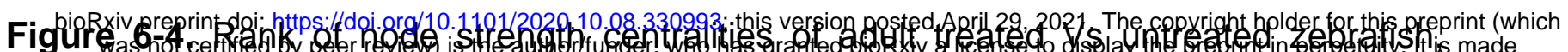

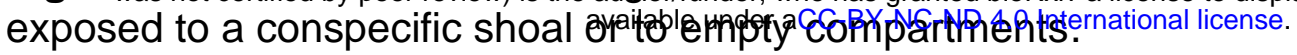

\begin{tabular}{ccccc} 
Rank & AE & AS & CE & CS \\
\hline 1 & DM & VD_a & VD_p & VV_p \\
2 & DL & VL & VC_p & DM \\
3 & VS & VV_a & VV_a & Dd \\
4 & VP & VM & VV_p & PPA \\
5 & VV_p & VV_p & Dd & VD_p \\
6 & VD_m & VP & VD_a & VC_p \\
7 & VL & VD_m & VC_a & VC_a \\
8 & Dd & VS & VD_m & VS \\
9 & PPp & VC_p & PPp & VP \\
10 & VD_p & VD_p & VL & DL \\
11 & VM & PPA & VM & VV_a \\
12 & VC_p & PPp & DL & VD_m \\
13 & VC_a & DL & DM & VM \\
14 & VD_a & VC_a & VP & VD_a \\
15 & VV_a & Dd & PPA & PPp \\
16 & PPA & DM & VS & VL \\
\hline
\end{tabular}

$A E$, early-MTZ treated fish exposed to empty compartment; $A S$, early MTZ-treated fish exposed to shoal; CE, untreated fish exposed to empty compartment; CS, untreated fish exposed to shoal; $V v \_a$, anterior part of the ventral nucleus of the ventral telencephalic area $(V) ; V v \_p$, posterior part of the ventral nucleus of the ventral telencephalic area $(\mathrm{V}) ; V d \_a$, anterior part of the dorsal nucleus of $\mathrm{V} ; V d \_m$, medial part of the dorsal nucleus of $\mathrm{V} ; \mathrm{Vd} \_p$, posterior part of the dorsal nucleus of $\mathrm{V} ; V c \_a$, anterior part of the central nucleus of $\mathrm{V} ; \mathrm{V} \_\_p$, posterior part of the central nucleus of $\mathrm{V} ; D m$, medial zone of the dorsal telencephalic area (D); DI, lateral zone of the dorsal telencephalic area (D); Dd, dorsal zone of the dorsal telencephalic area (D); $V s$, supracommissural nucleus of $\mathrm{V} ; \mathrm{Vp}$, postcommissural nucleus of $\mathrm{V} ; P p a$, anterior part of the parvocellular preoptic nucleus; Ppp, posterior part of the parvocellular preoptic nucleus; $V M$, ventromedial thalamic nucleus; $V L$, ventrolateral thalamic nucleus; 
bioRxiv preprint doi: https://doi.org/10.1101/2020.10.08.330993; this version posted April 29, 2021. The copyright holder for this preprint (which

was not certified by peer review) is the author/funder, who has granted bioRxiv a license to display the preprint in perpetuity. It is made available under aCC-BY-NC-ND 4.0 International license.

Figure 6-4. Rank of node strength centralities of adult treated vs untreated zebrafish, exposed to a conspecific shoal or to empty compartments. 